\title{
A Critical Review of the Use of Surfactant-Coated Nanoparticles in Nanomedicine and Food Nanotechnology
}

\author{
Taiki Miyazawa $\mathbb{D}^{\prime}$ \\ Mayuko Itaya ${ }^{2}$ \\ Gregor C Burdeos $\mathbb{D}^{3}$ \\ Kiyotaka Nakagawa ${ }^{2}$ \\ Teruo Miyazawa' \\ 'New Industry Creation Hatchery \\ Center (NICHe), Tohoku University, \\ Sendai, Miyagi, Japan; ${ }^{2}$ Food and \\ Biodynamic Chemistry Laboratory, \\ Graduate School of Agricultural Science, \\ Tohoku University, Sendai, Miyagi, Japan; \\ ${ }^{3}$ Institute for Animal Nutrition and \\ Physiology, Christian Albrechts University \\ Kiel, Kiel, Germany
}

\begin{abstract}
Surfactants, whose existence has been recognized as early as $2800 \mathrm{BC}$, have had a long history with the development of human civilization. With the rapid development of nanotechnology in the latter half of the 20th century, breakthroughs in nanomedicine and food nanotechnology using nanoparticles have been remarkable, and new applications have been developed. The technology of surfactant-coated nanoparticles, which provides new functions to nanoparticles for use in the fields of nanomedicine and food nanotechnology, is attracting a lot of attention in the fields of basic research and industry. This review systematically describes these "surfactant-coated nanoparticles" through various sections in order: 1) surfactants, 2) surfactant-coated nanoparticles, application of surfactant-coated nanoparticles to 3) nanomedicine, and 4) food nanotechnology. Furthermore, current progress and problems of the technology using surfactant-coated nanoparticles through recent research reports have been discussed.
\end{abstract}

Keywords: drug delivery system, drug targeting, food science, food packaging, nonionic surfactants, safety assessment

\section{Introduction}

Surfactants have been closely associated to humans for a long time, and these continue to be a necessity in our lives until now. The earliest report regarding the presence of surfactants is the record of soapy traces observed in clay cylinders at the Babylonian archeological site in Mesopotamia in 2800 BC. ${ }^{1,2}$ Sumerian tablets were excavated from the Mesopotamian archeological site in $2200 \mathrm{BC}$, and its cuneiform script describes how to make soap from animal fat and ash. ${ }^{1,2}$ Until the latter half of the 19th century, soap was reported to be the only artificial surfactant. However, in Germany after World War I, soap was found unsuitable for hard or acidic water, and its severe shortage prompted manufacturers to develop new surfactants to meet market demand, resulting in the development of miscellaneous surfactants. ${ }^{3}$ For example, the synthesis method of sodium dodecyl sulfate (SDS, also named as sodium lauryl sulfate [SLS]), one of the most produced and consumed surfactants until present, was first reported in Germany in 1933 (Figure 1). ${ }^{4}$ Surfactants have been widely used not only in adhesives, coatings, cosmetics, household detergents, industrial cleaning agents, oil field chemistry, paints, pesticides, plastics, textiles, but also in the fields of food and medicine. ${ }^{5}$ It was reported in the year 2000 that $4250 \mathrm{k}$ tons of detergent and 1190k tons of fabric softener was
Correspondence: Taiki Miyazawa New Industry Creation Hatchery Center (NICHe), Tohoku University, 6-6-10 Aramaki Aza Aoba, Aoba-Ku, Sendai, Miyagi, 980-8579, Japan

$\mathrm{Tel} / \mathrm{Fax}+8 \mathrm{I} 227953205$

Email taiki.miyazawa0II0@gmail.com 

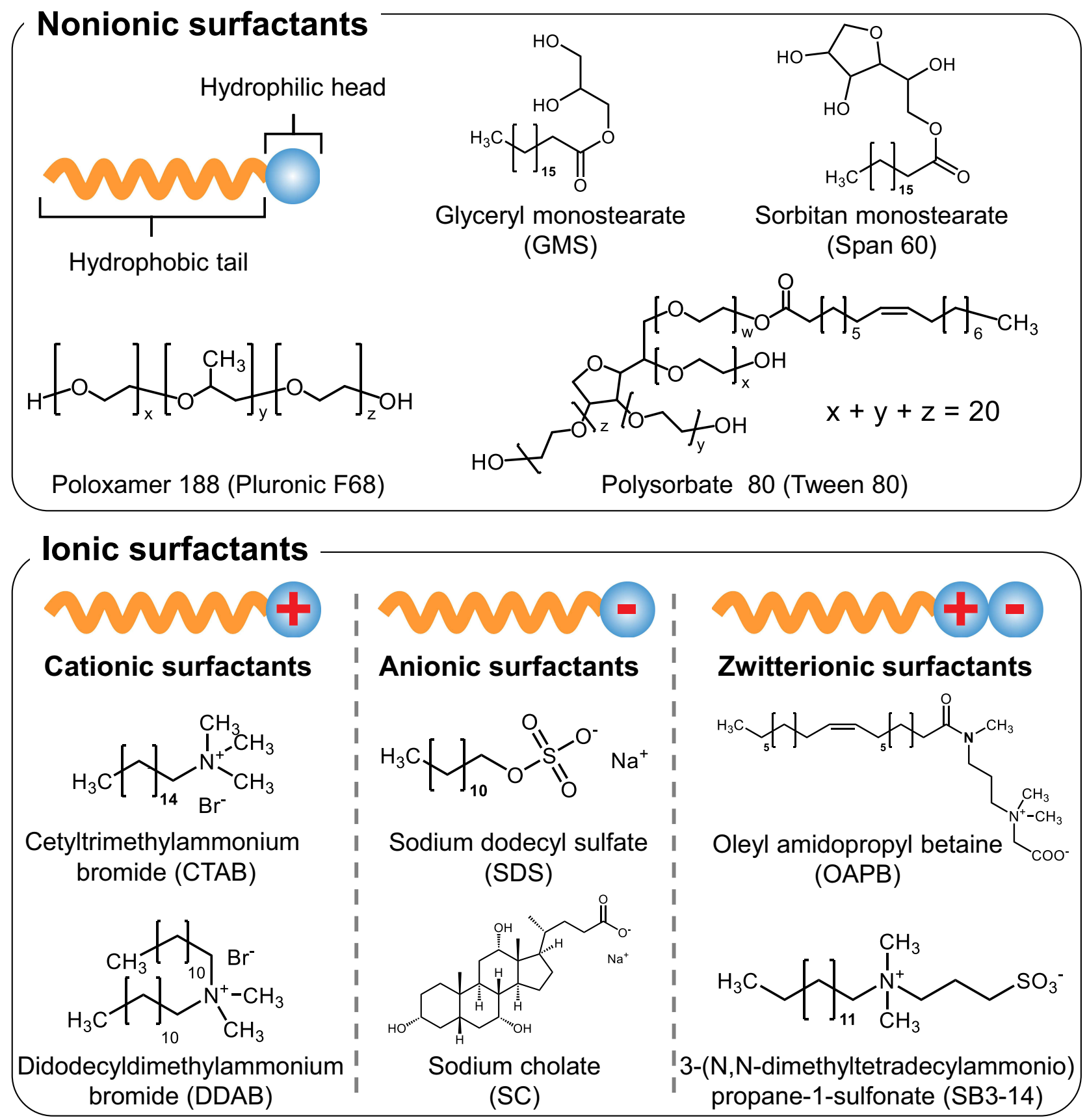

Figure I Classification of surfactants and structures of the ionic and nonionic surfactants mentioned in this review.

being consumed each year in Western Europe. ${ }^{6}$ The global consumption of household detergents in 2016 was 73.4 million tons. ${ }^{7}$ Due to its wide range of applications and high consumption, the global surfactant market was valued at $\$ 43,655$ million in 2017 and will reach approximately $\$ 64,408$ million by $2025 .{ }^{8}$ In other words, the compound's annual growth rate from 2018 to 2025 is expected to be $+5.4 \%{ }^{8}$
The concept of "Nanotechnology" was introduced by Richard Feynman in 1959 and named by Norio Taniguchi in 1974; this technology has been applied to nanoparticles, which are progressively being used in medicine and food industries and sometimes referred to as nanomedicine or food nanotechnology, respectively. ${ }^{9,10}$ Among them, surfactant-coated nanoparticles have been attracting attention in recent years because surfactants provide additional 
functions to nanoparticles. The present review is aimed to characterize the functions of nanoparticles provided by surfactants. The applications of surfactants, nanoparticles, and surfactant-coated nanoparticles in the field of nanomedicine and food nanotechnology along with some examples are included here. To systematically understand the relationship between the surfactants and nanoparticles, it is necessary to understand each of them individually. Therefore, this review introduces surfactants, surfactantcoated nanoparticles, and applications of surfactant-coated nanoparticles to nanomedicine and food nanotechnology. Through this review, we hope to visualize the current development and associated problems of surfactant-coated nanoparticles, bridge across disciplines, and lay the foundation for the development of new technologies.

\section{Method}

To carry out the literature search, Google Scholar, J-STAGE, MEDLINE, PubMed and Web of Science were employed. The search was based on key words such as surfactant and absorption/accelerated blood clearance/aggregation/aging/ Alzheimer's disease/antimicrobial/antioxidant/antiviral/artificial intelligence/bacteria/bioconcentration/blood-brain barrier/brain uptake/brain/cancer/cationic/cell membrane/cellular uptake/cholate/cholesterol/circulation/clinical trials/coating/ DLVO/daily meals/digestive system/drug/drug delivery system/EPR/emulsifiers/emulsion/environmental considerations/ environmental pollution/food nanotechnology/food packaging/food quality/food sensing/food technology/gold nanoparticles/health/history/hydrophilic/hydrophobic/inflammation/ inorganic/intravenous/ionic/liposomes/liquid/machine learning/medical/medicine/mucus layer/nanoparticles/nanotechnology/nanotoxicology/niosomes/nonionic/opsonization/oral/ organic/Ostwald ripening/oxidative stress/P-glycoprotein/ phospholipid/plant/poloxamer/polyethylene glycol/polymer/ polymeric nanocomposites/polyphenol/polysorbate/process/ quantum dots/reactive oxygen species/reticuloendothelial system/SPION/safety/self-assembly/senescence/side effect/silica nanoparticles/silver nanoparticles/smart food/stability/stealth effect/supramolecular structures/surfactant-coated nanoparticles/tissue distribution/toxicity/tween/Van der Waals forces/ vesicles/vitamin.

\section{Surfactant}

\section{Overview of Surfactant}

Surfactants, which is an abbreviation for "surface-active agents," are classified as amphiphilic compounds due to the presence of both hydrophilic and hydrophobic groups in their chemical structure. ${ }^{11}$ Depending on the characteristic of the hydrophilic group, surfactants can be broadly classified into four types: Cationic surfactants (positively charged hydrophilic groups), anionic surfactants (negatively charged hydrophilic groups), zwitterionic surfactants (having both positively and negatively charged hydrophilic groups), and nonionic surfactants (the hydrophilic group has no charge) (Figure 1). ${ }^{12}$ Cationic surfactants contain alkylamine or quaternary ammonium salts in their hydrophilic groups and can be adsorbed on negatively charged interfaces such as keratin (a component of skin and hair), natural fibers, and chemical fibers. They have antistatic and disinfectant properties, and are used as antistatic agents, coating agents, disinfectants, and softeners (hair conditioners and fabric softeners). Anionic surfactants contain carboxylic acid salts, sulfonates, sulfate salts, sulfate esters, or phosphates in their hydrophilic groups and offer good detergency, foaming property, foam stability, and penetration. They are used as foaming agents, paints, protein solubilizers, soaps, and present in various household and industrial detergents. Zwitterionic surfactants contain carboxy betaine, imidazolium betaine, aminoethylglycine salt, or amine oxide in their hydrophilic groups. They are often used as auxiliary materials to enhance the effectiveness of other surfactants or coexisting compounds. For example, anionic surfactant (sodium bis(2-ethylhexyl) sulfosuccinate [AOT], which self-assembles into the shape of ellipsoidal micelles), forms vesicles in the presence of zwitterionic surfactant (oleyl amidopropyl betaine [OAPB], which selfassembles into the shape of worm-like micelles [Figure 1]) and salt. ${ }^{13}$ Zwitterionic surfactant (3-[N,N-dimethyltetradecylammonio]propane-1-sulfonate [SB3-14] [Figure 1]) enhanced the loadability of natural flavonoid dye (quercetin) in wool and enhanced its antioxidant properties (Figure 1). ${ }^{14}$ Nonionic surfactants have non-dissociable chemical structures in their hydrophilic groups, such as amides, alcohols, esters, ethers, or phenols. They are used in cosmetics, as food emulsifiers, and skin cleansers due to low irritation and toxicity, which are the most important advantages associated with their application in nanomedicine and food nanotechnology. There are many reports available on the order of toxicity of surfactants, which generally demonstrate that cationic surfactants $>$ anionic surfactants $\geq$ zwitterionic surfactants $>$ nonionic surfactants, although toxicity may vary depending on the chemical structure. ${ }^{15-19}$ This is owing to the fact that the hydrophilic groups of nonionic surfactants do not ionize in aqueous 
solutions, and thus the critical micelle concentration of nonionic surfactants tends to be much lower than that of ionic surfactants. Therefore, they are less toxic than ionic surfactants. The hydrophobic groups of nonionic surfactants are composed of long-chain fatty acids and water-insoluble derivatives and are classified as fatty alcohols, esters, ethers, and block copolymers. ${ }^{20,21}$ Among the surfactants of one group, toxicity generally correlates with the ability of surfactant molecules to migrate from water to cell membranes.22 Therefore, the surfactant that has a longer chain length of the hydrophobic group and higher hydrophobicity can easily move to the lipid bilayer composed of phospholipids. Therefore, these are considered more toxic than highly hydrophilic surfactants. ${ }^{22}$ Henceforth, nonionic surfactants are most frequently used in the fields of nanomedicine and food nanotechnology. Currently, various nonionic surfactants are commercially available, so consumers can choose suitable compounds depending on their purpose.

In a system consisting of a single phase, surfactants are dispersed and equilibrated in the bulk. On the other hand, surfactants initiate their interactions after modification of various conditions such as electrolyte concentration, surfactant concentration, $\mathrm{pH}$, pressure, temperature, and type of solvent. This leads to supramolecular self-assembly of bilayer membrane vesicles, cylindrical micelles, lamellar phases, spherical micelles, etc. ${ }^{23}$ In addition, when the system consists of multiple phases, surfactants stabilize them due to their inherent physical characteristic of being localized at the interface (for example, air and water, oil and water, solid and water) due to their amphipathic chemical structure. In the absence of a surfactant, the molecules present in the respective aqueous and oil phases exert high surface tension due to intermolecular forces (such as hydrogen bonds), and the system is separated into different phases. When the surfactant is localized at the interface, an intermolecular force acts between the hydrophilic group of the surfactant and water molecule, thereby decreasing interfacial tension and surface tension leading to formation of supramolecular structures such as dispersed phase (such as emulsion) and continuous phase (such as bicontinuous liquid crystals) and a drastic change in the ratio of surface area to volume. The hydrophilic-hydrophobic balance (HLB, a parameter that indicates the surfactant's affinity for water and oil) and the critical packing parameter (CPP, a parameter that predicts the surfactant's self-assembly) are used to predict the properties of the surfactant. ${ }^{24-27}$ The "nanoscale supramolecular structures composed of surfactants" as discussed above, have been used as templates for the synthesis of inorganic materials ${ }^{28,29}$ enhancement of the activity of catalysts, ${ }^{30}$ reaction field of nanoreactors, ${ }^{31}$ modulation of wettability of biological interfaces, ${ }^{32}$ and enhanced oil recovery from heterogeneous rocks. ${ }^{33}$

\section{Nonionic Surfactants in the Pharmaceutical Industry}

The advantages of nonionic surfactants such as low cost, high stability, low toxicity, and amphiphilic nature can be used as next-generation materials and an alternative to applications of phospholipid-based nanostructures (hybrid lipid particles, nanocontainers, nanopores, and transistors). ${ }^{34}$ Due to these advantages, the field of nanomedicine is investigating the use of niosomes (vesicles composed of nonionic surfactants) instead of liposomes, which are composed of phospholipids and are widely used as carriers for drug and gene delivery. ${ }^{35}$ Bartelds et al prepared fluorophore (calcein)-encapsulating niosomes consisting of nonionic surfactants (polysorbate 80 [polyoxyethylene (20) sorbitan monooleate, also named as tween 80 [Figure 1]], sorbitan monostearate, and cholesterol). ${ }^{36}$ And compared their leakage to that of liposomes (consisting of phospholipids and cholesterol) after $25 \mathrm{~h}$ of incubation. The results showed that $10 \%$ of calcein leaked from the liposomes, whereas less than $3 \%$ of calcein leaked from the niosomes. This indicates that niosomes could retain the encapsulated material for a longer period. Puras et al prepared cationic niosomes consisting of cationic lipids and nonionic surfactants (polysorbate 80). ${ }^{37}$ They reported lower toxicity during transfection of cells with niosomes than with Lipofectamine ${ }^{\circledR}$, which is commonly used in gene transfer techniques. In addition, nonionic surfactants are widely used in protein drug delivery because they can stabilize proteins against interfacial tension and minimize the adsorption and aggregation of proteins at the interface. ${ }^{38,39} \mathrm{Li}$ et al demonstrated that the presence of nonionic surfactants (polysorbate 80 and poloxamer 188 [poly(ethylene glycol)-block-poly(propylene glycol)-block-poly(ethylene glycol), also named as pluronic F68] [Figure 1]) inhibited the irreversible adsorption of abatacept on silicone oil used as a lubricant for medical syringes with polysorbate 80 being more effective. ${ }^{40}$ Furthermore, poloxamers are used in more than $70 \%$ of commercially available monoclonal antibody drug delivery due to their ability to inhibit self-assembly and aggregation of antibodies. ${ }^{41-43}$ Moreover, the use of 
nonionic surfactants as pharmaceutical products is also being considered. For example, nonoxynol-9 has been found to have the potential for human immunodeficiency virus type 1 (HIV-1) infection and as topical disinfectant, but its efficacy has not been confirmed in clinical practice. $^{44,45}$

\section{Nonionic Surfactants in the Food Industry}

Approximately $75 \%$ of the total emulsifiers in the global food industry are mono- and di-glycerides, widely recognized as nonionic surfactants, either emulsifiers are industrially produced. ${ }^{46}$ Mono- and di-glycerides have been widely used as antimicrobial agents, antidegradants, emulsifiers, preservatives, and thickeners in food products such as beverages, ice cream, margarine, and shortening. Monoand di-glycerides are also reported to be present in trace amounts in natural food resources, such as paprika seed oil, pumpkin seed kernel oil, and watermelon seed kernel oil, which contain approximately $1 \%$ (proportion of total lipid) monoglycerides, and $0.3 \%$ (proportion of total lipid) diglycerides (Figure 1). ${ }^{47,48}$ The use of nonionic surfactants as food emulsifiers that enhance the absorption of fatsoluble food ingredients when taken orally is widely recognized. ${ }^{49}$ It is generally believed that emulsions have higher digestibility than other forms because they have more surface area to react with digestive enzymes, such as lipase. Salvia-Trujillo et al prepared emulsions with different particle sizes (small: $0.12 \mu \mathrm{m}$, medium: 0.19 $\mu \mathrm{m}$, and large: $14 \mu \mathrm{m}$ ) containing lipophilic food component ( $\beta$-carotene), and demonstrated the effect of particle size on its absorption using a gastrointestinal tract model. ${ }^{50}$ The results showed that the rate of digestion of lipids present in emulsions increased with decreasing particle size $($ small $=$ medium $>$ large) along with increased absorption of $\beta$-carotene (small $>$ medium $>$ large). To understand the mechanism of enhanced absorption, $\mathrm{Lu}$ et al prepared $\beta$-carotene encapsulated emulsions containing sunflower oil with monoglycerides in the range of $0-2 \%$ and demonstrated that the uptake of $\beta$-carotene into human colorectal adenocarcinoma (Caco-2) cells increased as the percentage of monoglycerides increased. ${ }^{51}$ They reported the mechanism of reduction in the surface charge of the emulsion in gastric fluid environment due to the presence of monoglycerides, which leads to an increase in the amount of lipase adsorbed onto the surface of the emulsion and reduction of creaming (a phenomenon in which thermodynamically unstable emulsions undergo phase separation over time). The antimicrobial effect of food ingredients is also known to be enhanced by coexistence with monoglycerides. Lee et al. found that the antimicrobial effect of linolenic acid on Bacillus cereus and Staphylococcus aureus was enhanced by coexistence with monoglycerides. ${ }^{52}$ They reported that the mechanism was that monoglycerides localized on the cell membrane of the bacteria enhanced the adhesion of linolenic acid to the cell membrane. Moreover, monoglycerides have been confirmed in synthesis-based scientific approaches to enhance the biological activity of food components. For example, omega-3 fatty acids are known to have useful physiological effects such as anti-inflammatory, antioxidant, anticancer, and anti-obesity and are available in the market as oral supplements, although they are known to be chemically unstable, difficult to dissolve in water, and have low absorption. To solve these problems and to enhance the physiological effects, eicosapentaenoic acidmonoglyceride, docosahexaenoic acid-monoglyceride, and docosapentaenoic acid-monoglyceride (in which eicosapentaenoic acid, docosahexaenoic acid, docosapentaenoic acid were esterified and bound to the sn- 1 position of the glycerol moiety in the monoglyceride structure) have been studied. ${ }^{53,54}$ In addition to these applications, nonionic surfactants are used in a variety of applications in the food industry and are detailed in other reviews. ${ }^{55,56}$

\section{Surfactant-Coated Nanoparticles}

Nanoparticles have been used in the fields of nanomedicine and food nanotechnology to impart a variety of functions to encapsulated compounds. However, depending on the surface structure, the prepared nanoparticles are difficult to disperse in water and be aggregated in a short time. An approach to solve this problem is to allow coexistence of the prepared nanoparticles and the surfactant so that the surface of the nanoparticles is covered with the surfactant, and the nanoparticles are stabilized in the system. These nanoparticles are called "Surfactant-coated nanoparticles" (Figure 2A) ${ }^{57,58}$ It is important to understand the interaction between the nanoparticles and the surfactant in surfactant-coated nanoparticles for their efficient performance.

The nanoparticles exert forces on each other. Orientation interactions (Keesom interactions), dipole interactions (Debye interactions), and dispersion interactions (London interactions) are collectively called "van der Waals forces" and are responsible for intermolecular interactions. ${ }^{59}$ Van der Waals forces acting between particles are considered to be caused by the attractive forces 


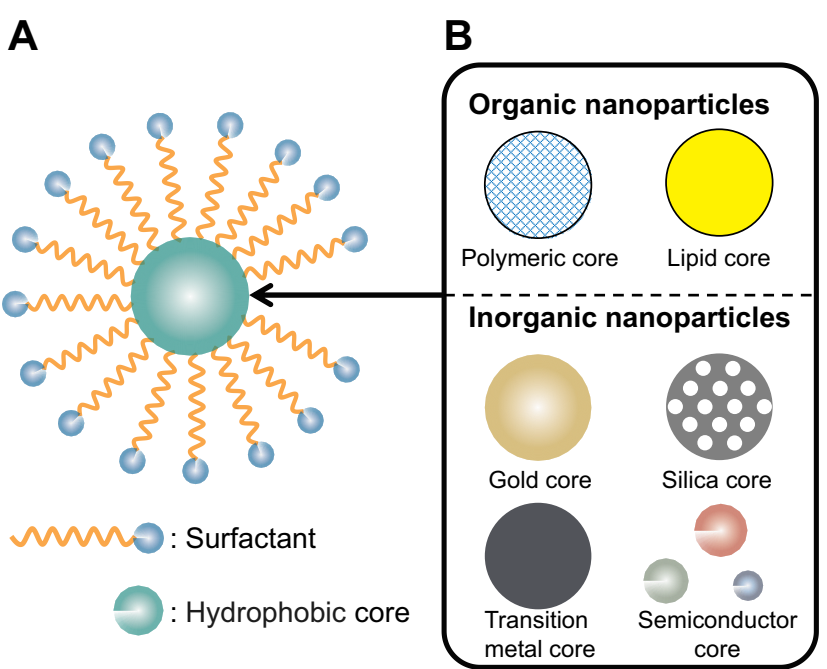

Figure 2 (A) Typical Illustration of surfactant-coated nanoparticles. (B) Various organic and inorganic materials used in the core of surfactant-coated nanoparticles.

between the molecules present in each particle and are expressed by the equation (Figure $3 \mathrm{~A}(1)$ ), ${ }^{60}$ where $A_{H}$ is the Hamaker constant that varies depending on the type of molecules present in the particles, for example, the value of Hamaker constant is $6.5 \times 10^{-20} \mathrm{~J}$ for poly (lactic-coglycolic acid) (PLGA), and in the range of $0.9-3.0 \times 10^{-19} \mathrm{~J}$ for gold. ${ }^{61,62}$ As the size, shape, and temperature of metallic particles change, the value of the Hamaker constant also changes due to the change in the dielectric constant. ${ }^{62}$ The above equation is approximated to the equation (Figure 3A (2)) by the Derjaguin approximation by assuming that the distance between the two particles is narrower than the radius (Figure 3B). ${ }^{63}$ It can be inferred from these equations that the van der Waals force between particles becomes weaker as the distance between the particles increases and becomes stronger as the size of the particles increases.

The surface charge between particles is also important as it determines the electrostatic repulsive force. Particles that are positively or negatively charged in solution form an ionic atmosphere due to the attraction of counter ions to the surface of these particles, resulting in the formation of an electric double layer. When particles come close to each other, the overlap of the electric double layer leads to a change in the ion concentration and the repulsive force is
A

$$
\begin{aligned}
& V_{A}=-\frac{A_{H}}{12 \pi h^{2}} \text { (1) } \quad V_{A}=-\frac{A_{H} R}{12 h} \\
& V_{R}=\frac{64 \pi R n k T \gamma^{2}}{\kappa^{2}} e^{-\kappa h}=\frac{G \kappa R k T}{12} e^{-\kappa h} \\
& V=V_{A}+V_{R}=\frac{A \kappa R}{12}\left(\frac{G k T}{A} e^{-\kappa h}-\frac{1}{\kappa h}\right) \\
& \kappa=\sqrt{\frac{2 z^{2} e^{2} n}{\varepsilon_{r} \varepsilon_{0} k T}}(5) \\
& G=\frac{12 \times 64 \pi \gamma^{2} n}{\kappa^{3}}=\frac{384 \pi \gamma^{2} \varepsilon_{r} \varepsilon_{0} k T}{(z e)^{2} \kappa}
\end{aligned}
$$

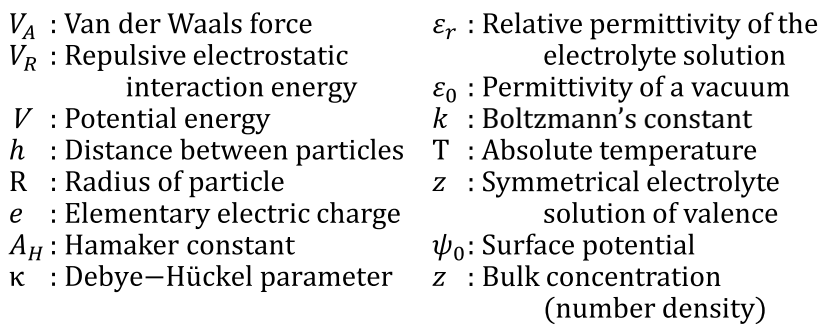

B

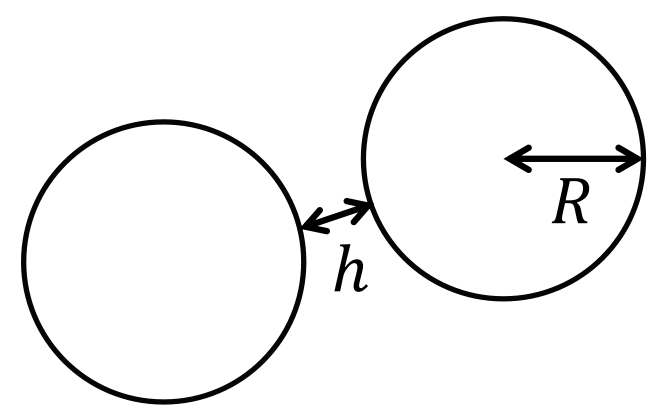

C

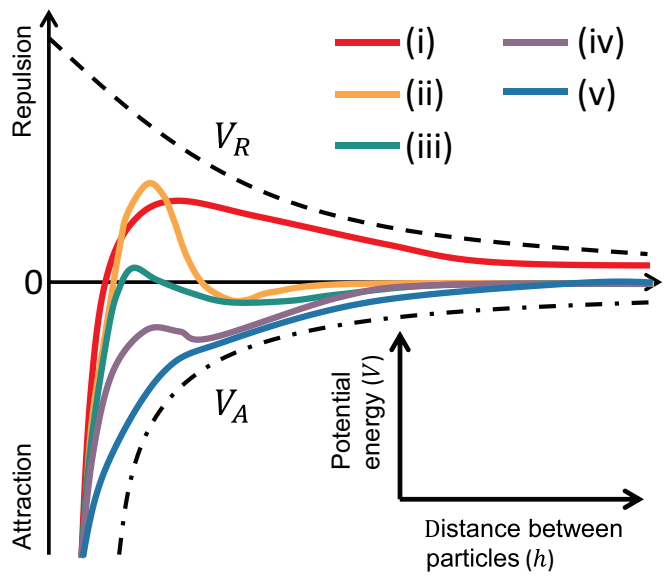

Figure 3 (A) Equations of the DLVO theory. (B) Relationship between two particles assuming the DLVO theory. (C) A typical example of potential energy presented in the DLVO theory.

Notes: (A) Data from Hamley ${ }^{63}$ and Ohshima. ${ }^{65}$ 
generated. This phenomenon is called "electrostatic repulsive force." ${ }^{, 2263}$ Models of the strength of this electrostatic repulsive force include Helmholtz, Gouy-Chapman, Stern, and BDM (Bockris/Devanathan/Muller), each of which has been formulated. ${ }^{64}$ One of the most widely used equations for the electrostatic repulsive force is for colloidal systems with dispersed nanoparticles (Figure 3A (3)). ${ }^{65}$ The theory that considers both the "attractive force" of the van der Waals interaction and the "repulsive force" of the electrostatic repulsion is called the Derjaguin-LandauVerwey-Overbeek (DLVO) theory. ${ }^{63,66,67}$ DLVO theory is named after the scientists who contributed to its development and can explain the coagulation/dispersion state of particles. In DLVO theory, the stability of a colloid is defined as the total sum of van der Waals force and electrostatic repulsion force between particles, and the total potential energy and is expressed as shown in Figure 3A (4-7). ${ }^{62,65,68}$

A typical example of the DLVO theory is shown in Figure $3 C^{60,63,65}$ At high potential energy, the particles are stable because they repel each other (Figure 3C (i)). As shown in Figure 3C (ii), if there is a deep secondary minimum, the particles are in a stable equilibrium state. As shown in Figure 3C (iii), when the secondary minimum is shallowly declined the particles gradually aggregate. As shown in Figure 3C (iv) and (v), if the attractive van der Waals force is stronger than the electrostatic repulsive force, the particles will aggregate in a short time. To elucidate the influence of surfactant adsorption on the aggregation behavior of nanoparticles, Farrokhbin et al dispersed three types (amidine latex, silica, and sulfate latex) of nanoparticles in non-polar solvent (decane) and added an anionic surfactant (SDS) and assessed the parameters for aggregation based on the DLVO theory (shielding distance, surface charge, and van der Waals force) ${ }^{69}$ As a result, they reported an increase in inhibition of aggregation and stabilization of dispersion in a concentration-dependent manner until the concentration of anionic surfactant in the system reached a certain concentration. Espinosa et al also reported that the dispersion of poly (methyl methacrylate) nanoparticles was stabilized in nonpolar solvents (hexane) when a nonionic surfactant (sorbitan trioleate, also named as Span 85) was present in the system. ${ }^{70}$ Although, the DLVO theory is adaptable to particles of hard materials, however, there are limitation in its applicability to soft materials such as cells and lipoproteins, causing different dispersion phenomena in vivo. $^{71,72}$ Therefore, additional theory will need to be developed. If there is a difference in the size of the particles in the system, the smaller particles are incorporated into the larger particles over time due to the difference in their surface energies, with the larger particles becoming larger and the smaller particles disappearing from the system. This phenomenon is known as "Ostwald ripening" and is widely recognized as a principle that applies to all organic and inorganic particles. ${ }^{73}$ As mentioned at the beginning of this section, particle agglomeration is a concern in particle dispersion systems. However, it has been reported that the presence of a surfactant in the system induces it to get adsorbed to the surface of the particles and lowers the speed of ripening by several orders of magnitude. ${ }^{74}$ Kiss et al demonstrated the mechanism of adsorption of nonionic surfactants (pluronic PE6100, PE6400 and PE6800) on hydrophobic interfaces (blend film composed of polylactic acid [PLA] and PLGA). ${ }^{75}$ They reported that highly hydrophilic surfactants could not adsorb to the hydrophobic interface, while surfactants with both high and low hydrophilic moieties could adsorb and distribute effectively to the hydrophobic interface. The presence of surfactants at the solid interface increased steric stabilization. When the nanoparticles come close to each other, the hydrophobic groups of the surfactant on the surface of the nanoparticles limit the mutual penetration, resulting in steric hindrance and stabilization. Steric stabilization is different from electrostatic repulsive forces, such as being unaffected by the electrolyte concentration of the solvent and adaptable over a wide range of nanoparticle concentrations. ${ }^{76}$ Santander-Ortega et al studied in detail the adsorption and stabilization mechanism of nonionic surfactants on PLGA nanoparticles and confirmed that a nonionic surfactant (poloxamer 188) adsorbed to the interface of PLGA nanoparticles when both of them coexisted. ${ }^{77}$ Moreover, they demonstrated that as the adsorption of nonionic surfactant (poloxamer 188) on the surface of PLGA nanoparticles increased, the steric stability of nanoparticles was greatly increased by the polyethylene oxide framework in nonionic surfactant, and the parameters of the DLVO theory indicated the formation of a stable dispersion. Furthermore, surprisingly, they found that the stabilization mechanism is not only explained by the DLVO theory and steric stabilization, but also dependent on the repulsive hydration forces to the hydrophilic interface constructed by nonionic surfactant (poloxamer 188 ) on the surface of nanoparticles. Since the stabilization by the repulsive hydration force is unaffected by the external salt concentration, the system is also expected to 
be stable in vivo and is attracting attention. Chaudhari et al and other researchers reported that an anionic surfactant (SDS) and a nonionic surfactant (poloxamers) had little effect on the release rate of the encapsulated drug from the solid dispersions. ${ }^{78-81}$ Conclusively, surfactants play an important role in the stabilization of nanoparticles and there are many applications of surfactant-coated nanoparticles as explained in the next section.

\section{Application of Surfactant-Coated Nanoparticles in Nanomedicine}

\section{Nanomedicine}

Nanotechnology is defined as the deliberate design, characterization, production, and application of materials, structures, devices, and systems by controlling their size and shape within the nanoscale range. ${ }^{82}$

Nanomedicine is regarded as "the use of nanoscale material properties and physical characteristics for the diagnosis and treatment of diseases at the molecular level". 82 According to a report of 2013, 789 clinical trials were in progress at 241 companies and research institutions, and 363 nanomedicine products and applications were identified. ${ }^{83}$ In the field of nanomedicine, surfactants are often used to impart new functions to nanoparticles. Recent progress in this field is summarized in Table 1. In the following sections, nanomedicine based on surfactantcoated organic and inorganic nanoparticles will be presented and the properties each nanostructure will be discussed separately. However, in practice, most approaches combine multiple materials and properties rather than only one. Therefore, in the field of nanomedicine, it is necessary to have integrated knowledge and approaches that are not limited to the respective organic and inorganic fields of expertise.

\section{Surfactant-Coated Organic Nanoparticles in Nanomedicine}

During disease treatment, the administered molecule (drug) can exhibit a therapeutic effect only when it reaches the target site of action, such as an area of inflammation or a cancer tissue. However, when a free drug is administered into the bloodstream, its therapeutic efficacy is severely limited due to various problems including premature degradation, expulsion of the drug due to the reticuloendothelial system (RES, also called the mononuclear phagocyte system [MPS]), degradation due to instability of the drug, poor dispersibility, and poor accumulation at the site of action. The resulting non-selective tissue distribution of drugs is a major factor responsible for drug toxicity (for example, dose-limiting toxicity [DLT]). ${ }^{84}$ Organic nanoparticles, which are widely used in the field of nanomedicine, have potential to overcome the above problems because they can impart a variety of advantages to the encapsulated substances. ${ }^{85}$ For example, organic nanoparticles encapsulating anticancer drugs, genes, and proteins can be delivered selectively to the target site of action or specific cells while protecting the encapsulation from degradation and RES; such a technology increases therapeutic efficacy and reduces side effects and is called a "drug delivery system". ${ }^{86-88}$ The constituents of the organic nanoparticles used in the drug delivery system are selected to be non-toxic or low-toxic to living organisms, and typical examples include biodegradable polymers (chitosan, gelatin, hyaluronic acid, PLGA, poly [alkyl cyanoacrylate], and poly-e-caprolactone), solid lipids (cetyl palmitate, cholesterol, palmitic acid, stearic acid, and tristearin), and proteins (albumin, collagen, gliadin, legumin, protamine and silk) (Figure 2B). ${ }^{89-91} \mathrm{~A}$ number of methods for preparing organic nanoparticles have been reported, and the related mechanism has been reviewed in detail by Anton et al. ${ }^{74}$ For example, the emulsification solvent evaporation technique (polymerand lipophilic drug-containing organic solvent is dispersed in surfactant-containing water to form an oil in water $[\mathrm{O} /$ W] emulsion as a template of nanoparticles, and then evaporated to precipitate polymeric nanoparticles containing the drug dispersed into the system) is widely used to prepare nanoparticles composed of biodegradable polymers, and the microemulsion method (oil phase containing low melting temperature lipid and lipophilic drug is dispersed in surfactant-containing water to form $\mathrm{O} / \mathrm{W}$ microemulsion as a template of nanoparticles, which is then rapidly cooled to precipitate drug-containing solid lipid nanoparticles) is widely used to prepare nanoparticles composed of solid lipids. ${ }^{90,92}$ The preparation of PLGA nanoparticles by solvent evaporation technique is widely used, and the most commonly used surfactant in the preparation process is polyvinyl alcohol (PVA). ${ }^{93}$ Pisami et al used three different surfactants (PVA, sodium cholate [SC] [Figure 1], sodium taurocholate [TC], [Figure 1]) in the preparation of PLGA nano/micro particles encapsulating lipophilic substances (perfluorooctyl bromide [PBOB]) by solvent evaporation technique (dichloromethane was used as the organic phase) and compared their detail of precipitation process by optical microscopy, confocal 


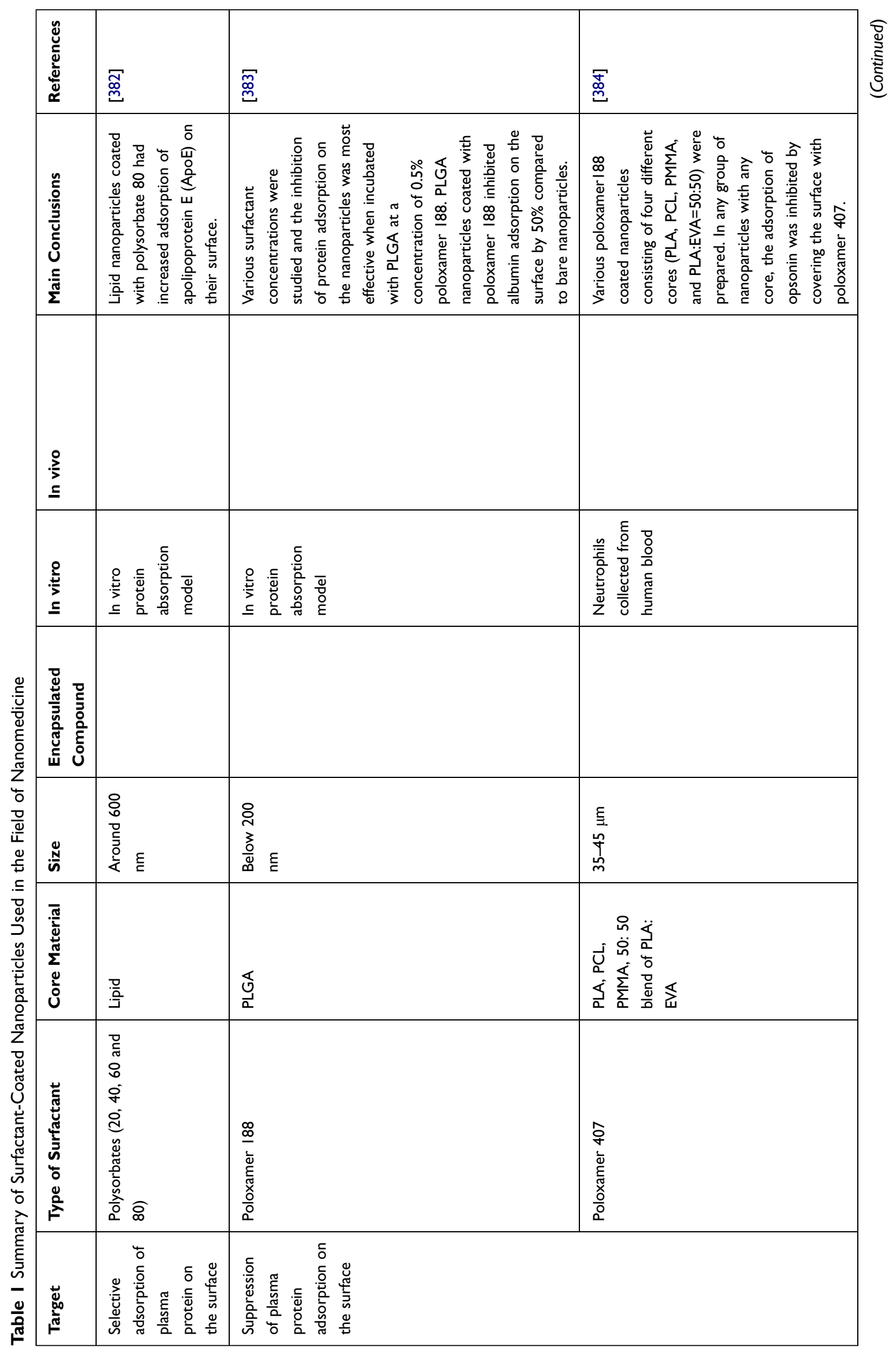




\begin{tabular}{|c|c|c|c|}
\hline 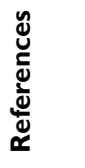 & $\stackrel{\square}{\varrho}$ & 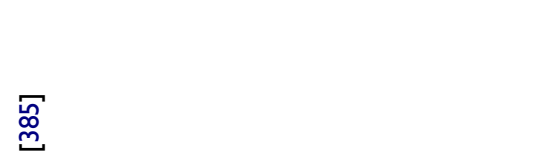 & 离 \\
\hline 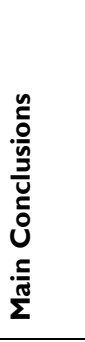 & 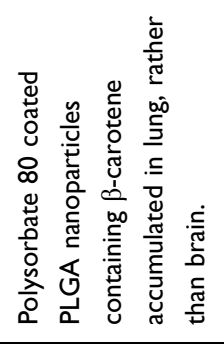 & 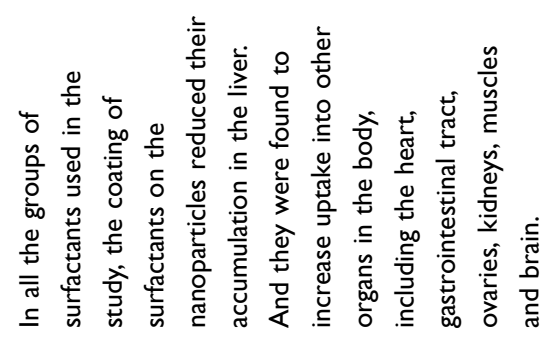 & 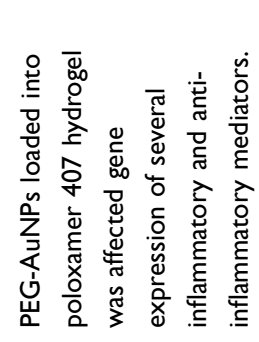 \\
\hline $\begin{array}{l}\stackrel{0}{>} \\
\underline{\underline{y}}\end{array}$ & 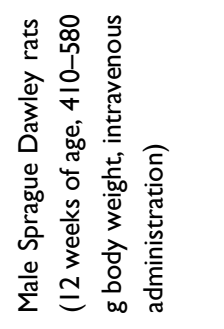 & 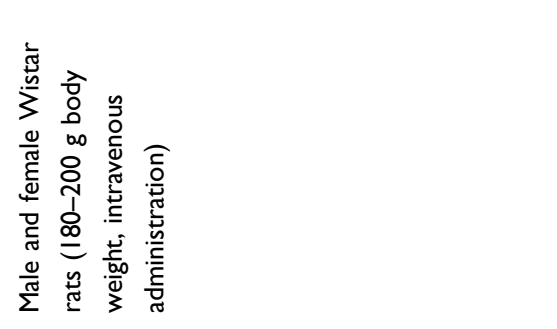 & 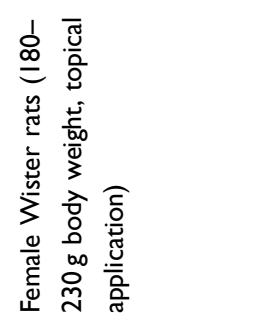 \\
\hline 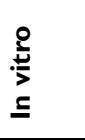 & & & \\
\hline 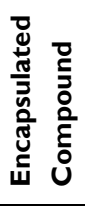 & 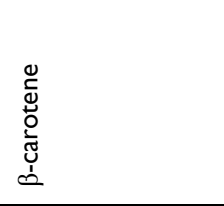 & & \\
\hline$\stackrel{N}{n}$ & 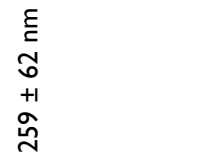 & $\begin{array}{l}\varepsilon \\
\vdots \\
0 \\
+1 \\
\underline{m}\end{array}$ & 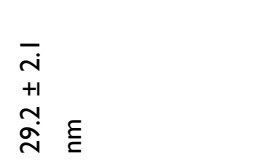 \\
\hline 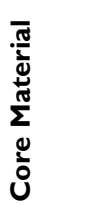 & 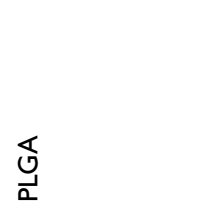 & 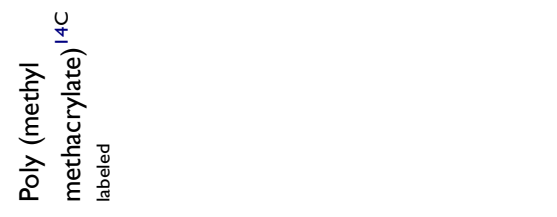 & $\begin{array}{l}\frac{0}{0} \\
0\end{array}$ \\
\hline 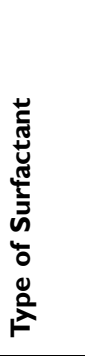 & 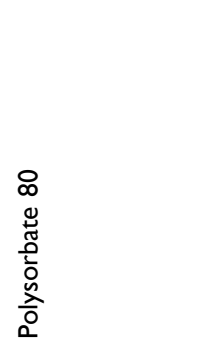 & 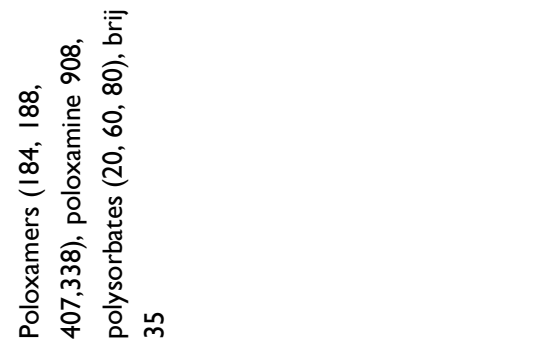 & 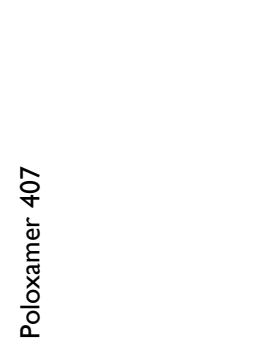 \\
\hline 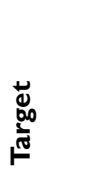 & 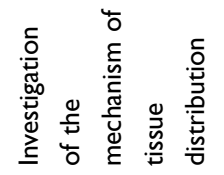 & & 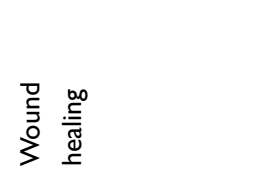 \\
\hline
\end{tabular}




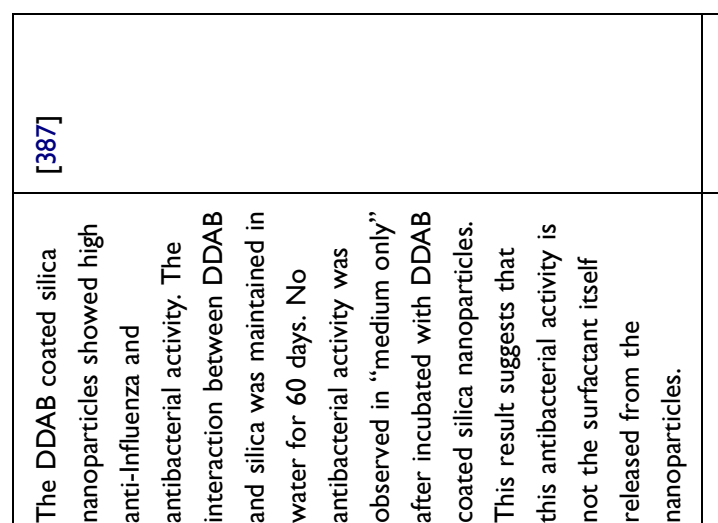

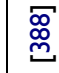
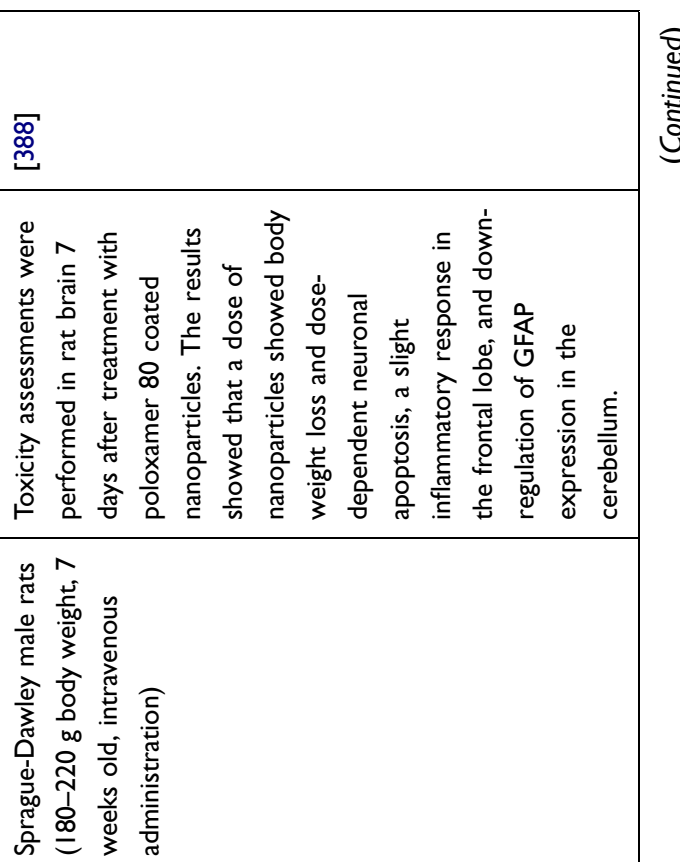

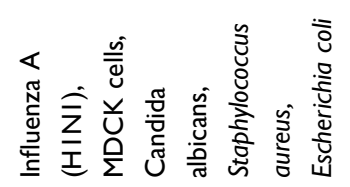

\begin{tabular}{|c|c|}
\hline & 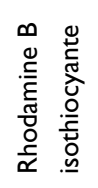 \\
\hline 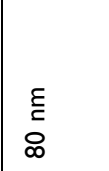 & $\begin{array}{l}\underline{E} \\
\underline{\underline{n}} \\
\underline{n} \\
\stackrel{+1}{n}\end{array}$ \\
\hline$\frac{\widetilde{\Xi}}{\overline{\bar{n}}}$ & 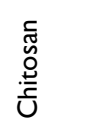 \\
\hline 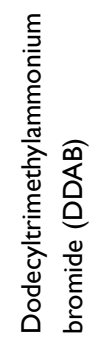 & 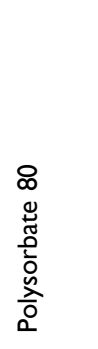 \\
\hline 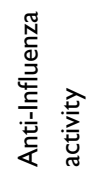 & 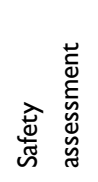 \\
\hline
\end{tabular}




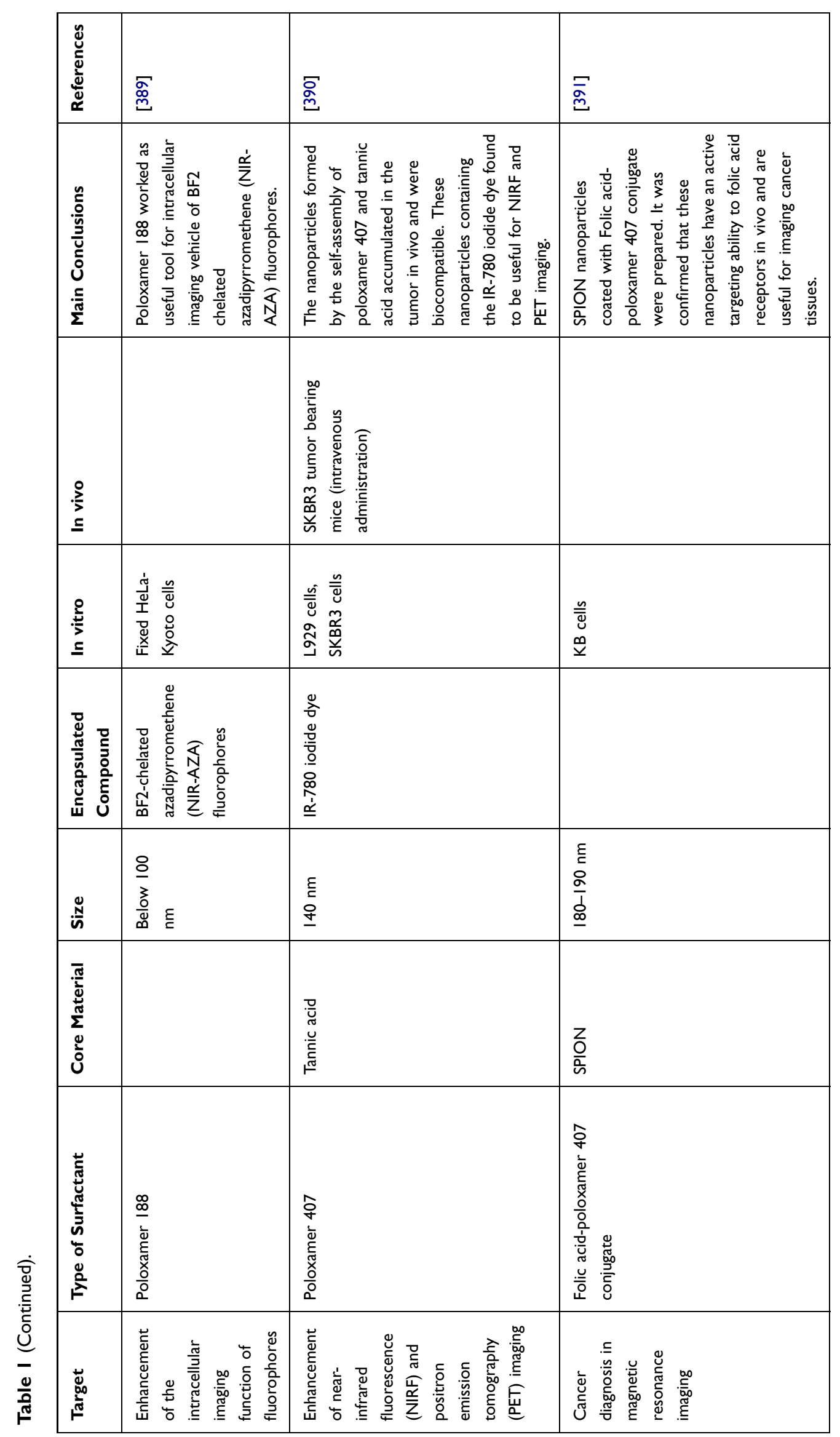




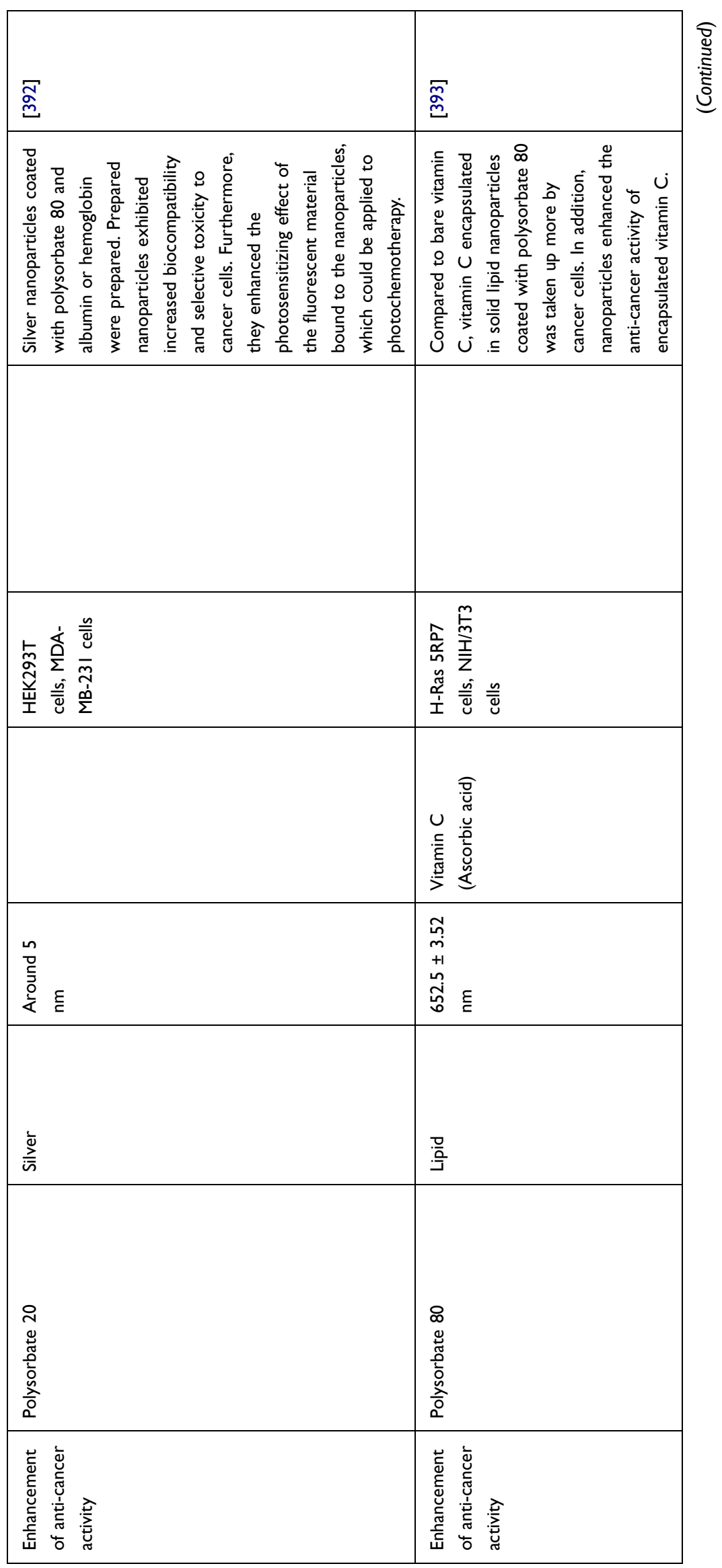




\begin{tabular}{|c|c|c|c|}
\hline 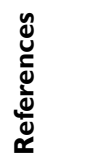 & $\begin{array}{l}F \\
\stackrel{F}{g}\end{array}$ & $\stackrel{\sqrt[\Omega]{\alpha}}{\underline{n}}$ & 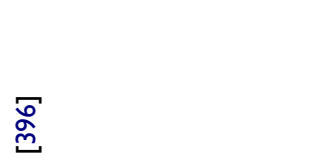 \\
\hline 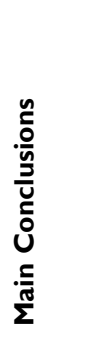 & 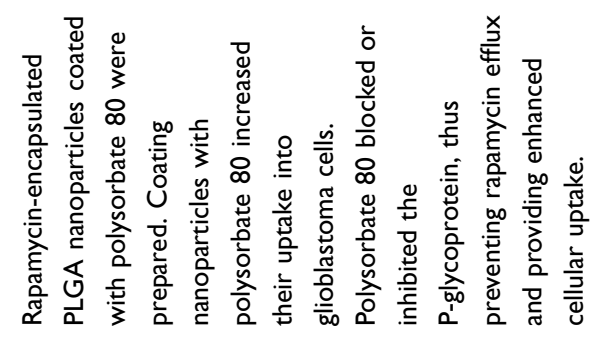 & 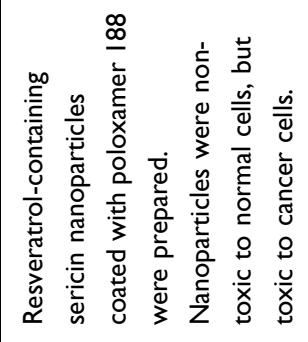 & 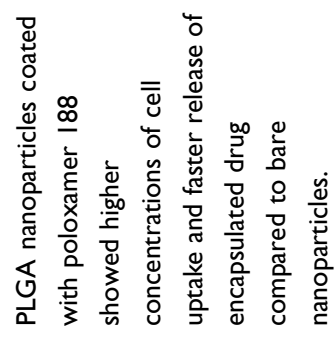 \\
\hline $\begin{array}{l}\stackrel{0}{>} \\
\underline{\underline{z}}\end{array}$ & & & \\
\hline 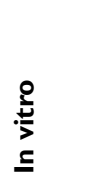 & $\begin{array}{l}\underline{\underline{\underline{E}}} \\
\stackrel{0}{U}\end{array}$ & 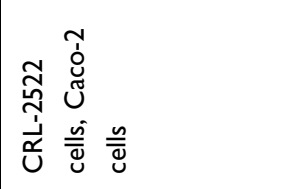 & 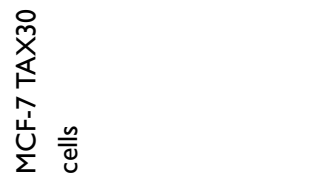 \\
\hline 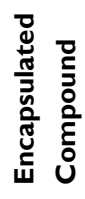 & 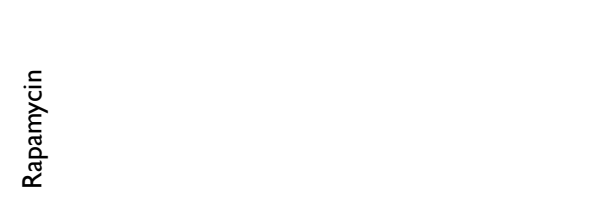 & 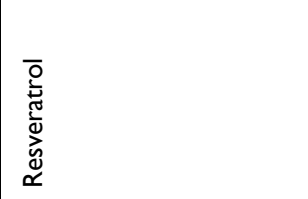 & 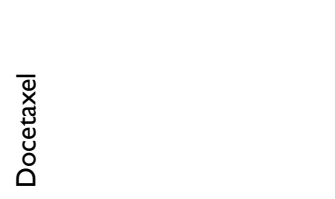 \\
\hline$\frac{N}{n}$ & $\begin{array}{l}\frac{E}{\Xi} \\
\frac{+1}{+} \\
\stackrel{+}{d}\end{array}$ & 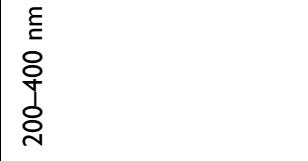 & $\begin{array}{l}0 \\
\infty \\
\infty \\
+1 \\
0 \\
\stackrel{\sim}{N} \\
\stackrel{N}{E}\end{array}$ \\
\hline 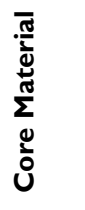 & 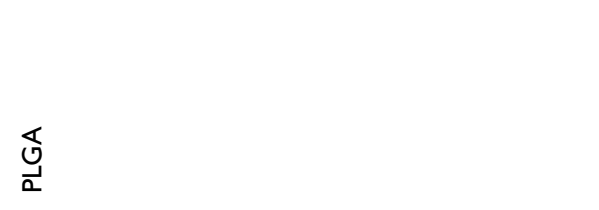 & 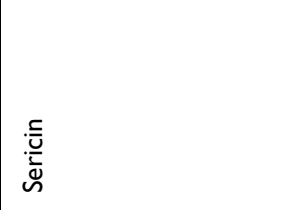 & 志 \\
\hline 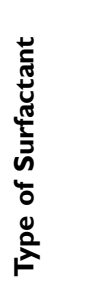 & 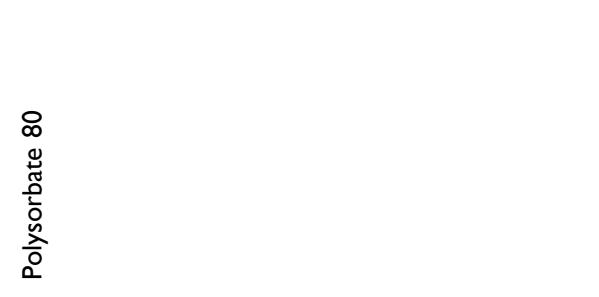 & 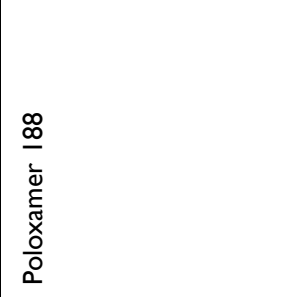 & 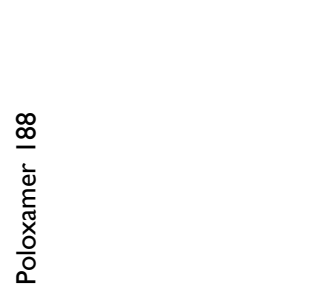 \\
\hline 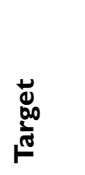 & 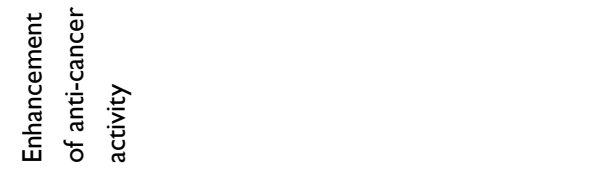 & 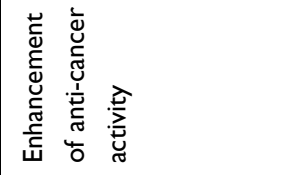 & 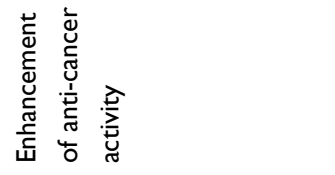 \\
\hline
\end{tabular}




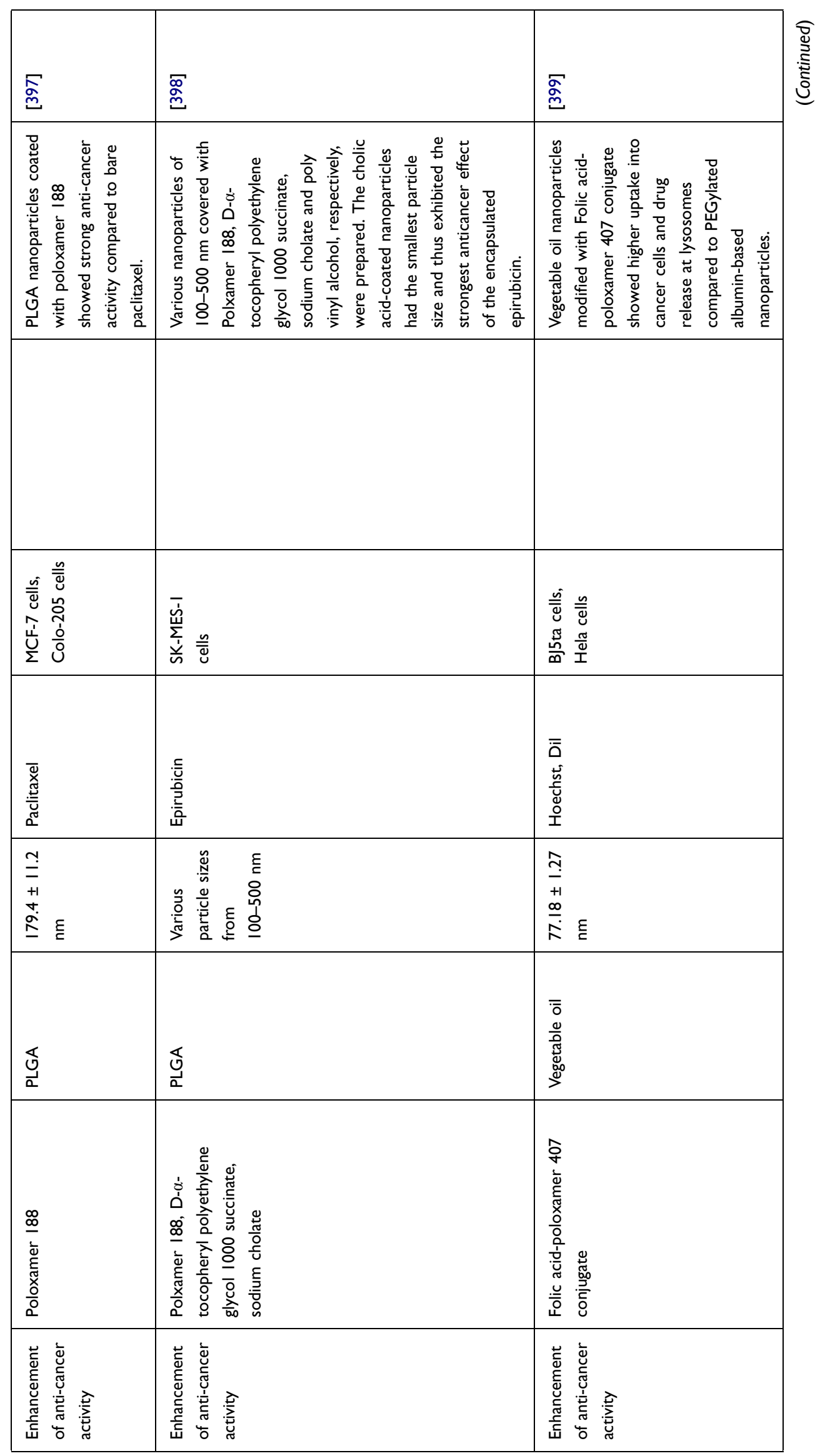




\begin{tabular}{|c|c|c|c|}
\hline 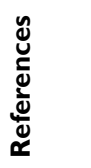 & 定 & $\overline{\bar{g}}$ & $\widetilde{\widetilde{\sigma}}$ \\
\hline 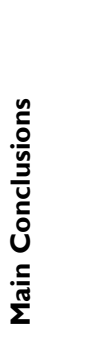 & 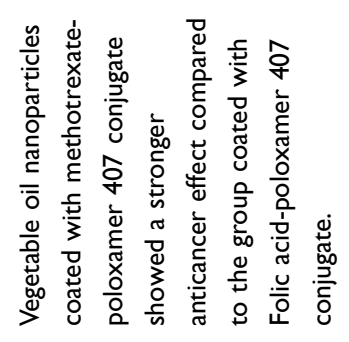 & 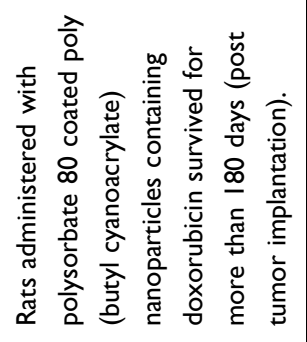 & 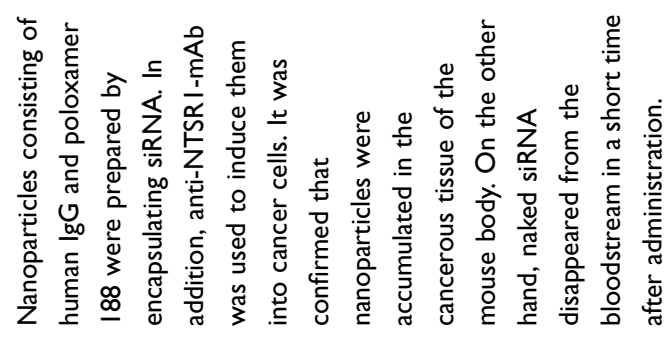 \\
\hline $\begin{array}{l}\stackrel{P}{>} \\
\underline{\underline{\underline{y}}}\end{array}$ & & 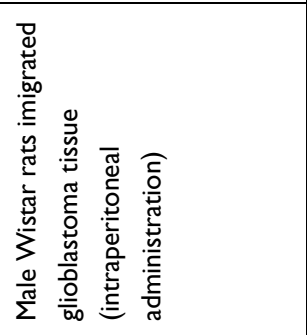 & 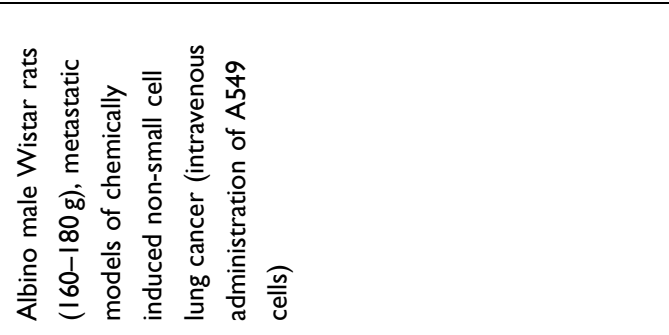 \\
\hline 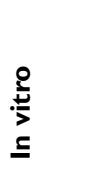 & 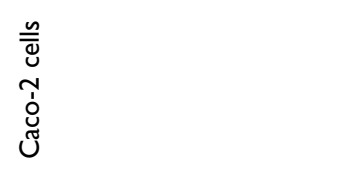 & & \\
\hline 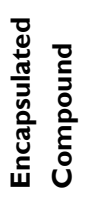 & 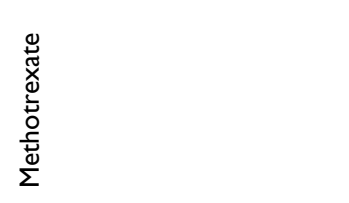 & $\begin{array}{l}\frac{5}{0} \\
\frac{0}{0} \\
\frac{0}{0} \\
0 \\
0 \\
0\end{array}$ & \\
\hline$\stackrel{N}{n}$ & $\begin{array}{l}\underset{+}{+} \\
\dot{N} \\
+1 \\
m \\
\dot{m} \\
\sigma\end{array}$ & 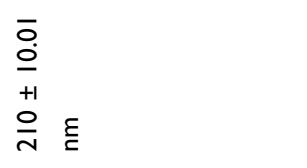 & 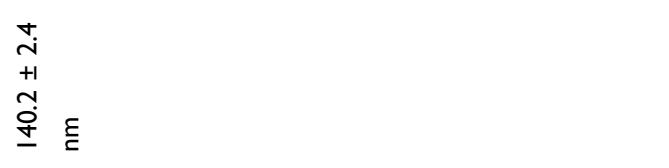 \\
\hline 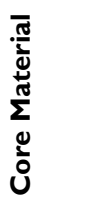 & 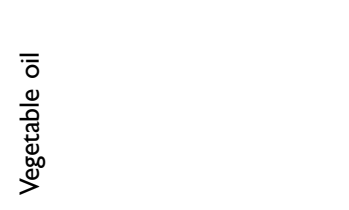 & 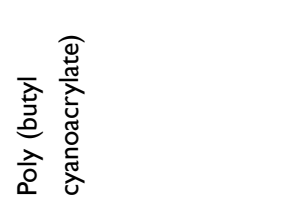 & 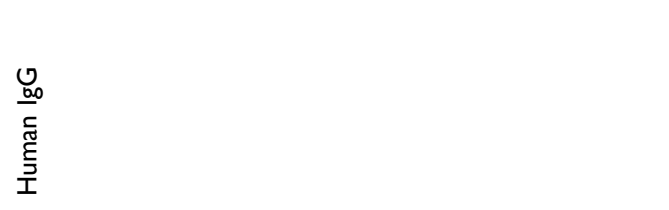 \\
\hline 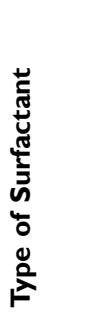 & 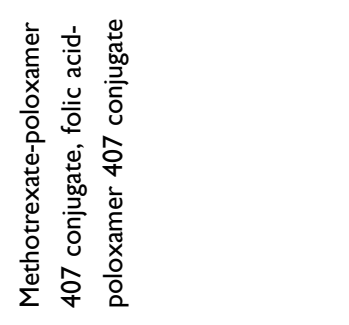 & 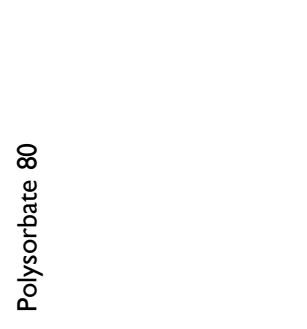 & 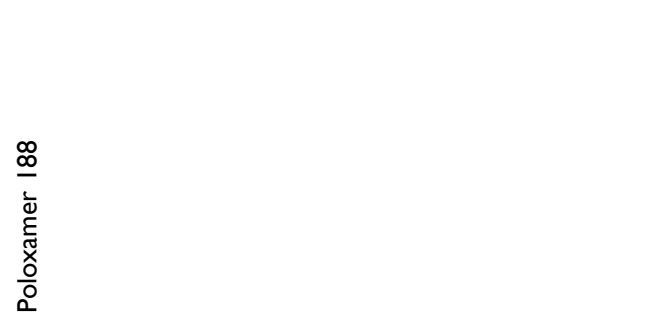 \\
\hline 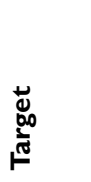 & 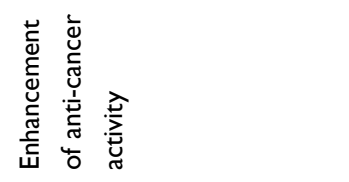 & 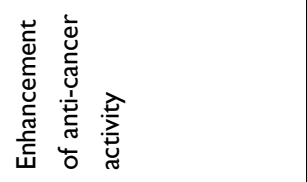 & 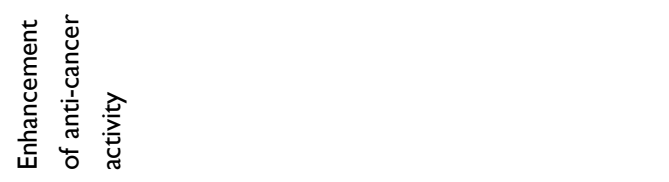 \\
\hline
\end{tabular}




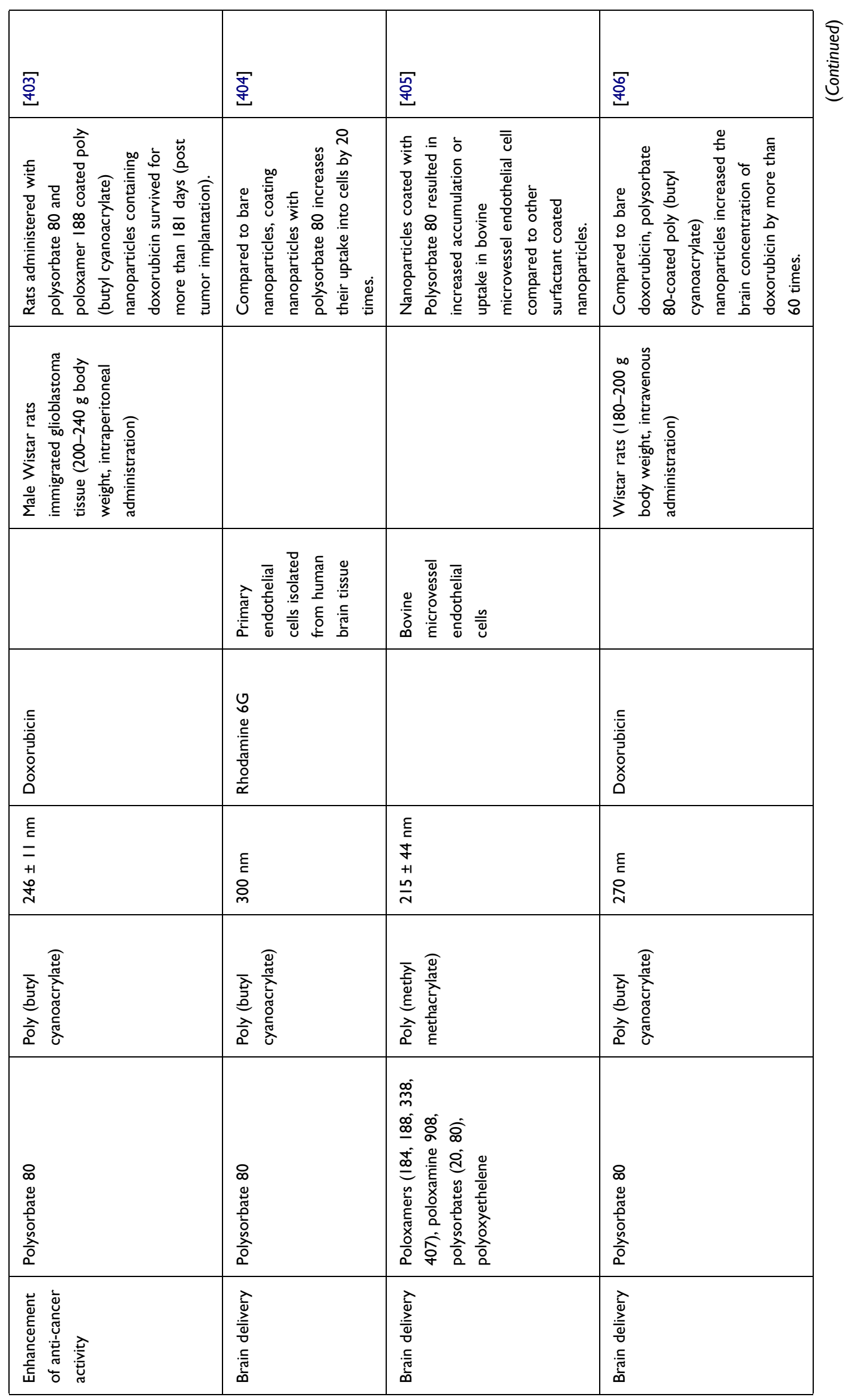




\begin{tabular}{|c|c|c|c|}
\hline 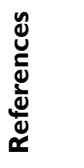 & $\underset{⿱ 乛}{+}$ & 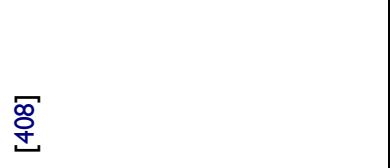 & 定 \\
\hline 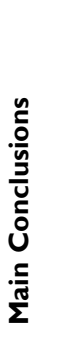 & 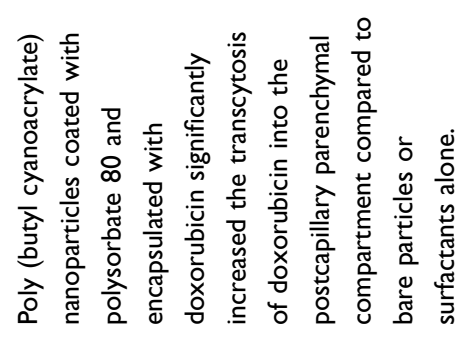 & 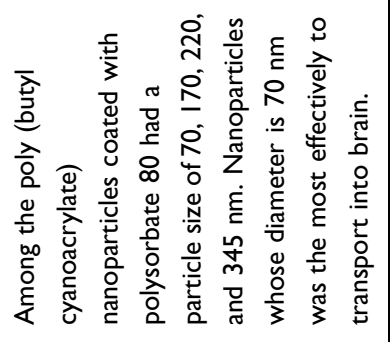 & 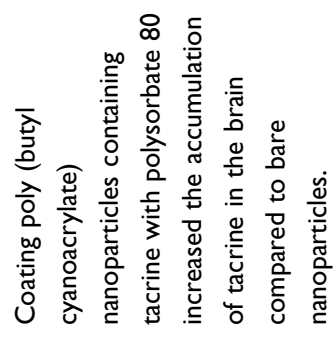 \\
\hline $\begin{array}{l}\stackrel{0}{>} \\
\underline{\underline{x}}\end{array}$ & 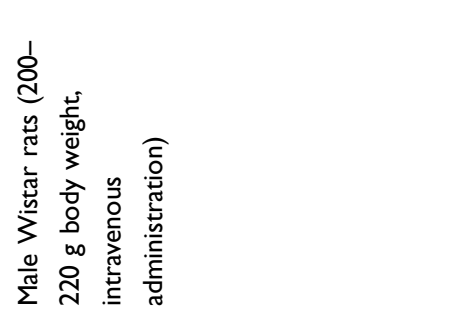 & 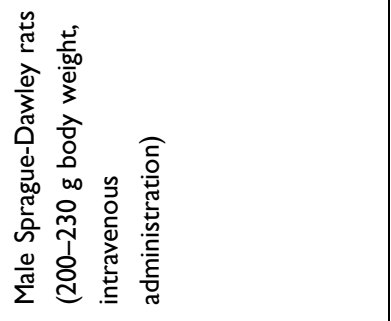 & 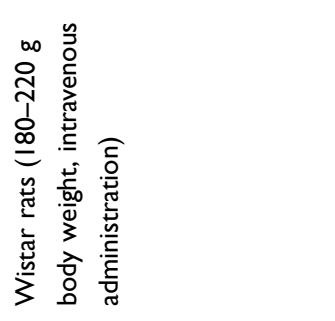 \\
\hline 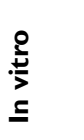 & & & \\
\hline 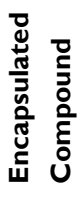 & 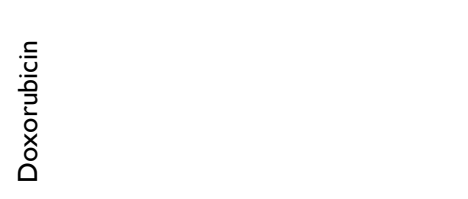 & 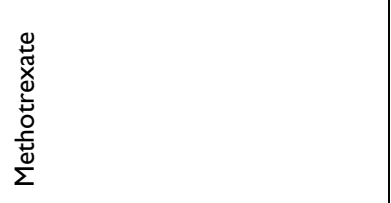 & 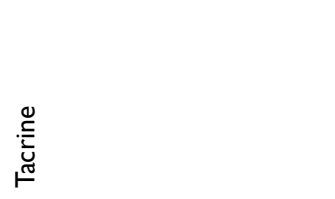 \\
\hline$\stackrel{\tilde{N}}{\tilde{n}}$ & 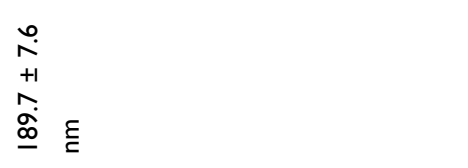 & 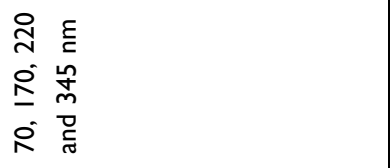 & 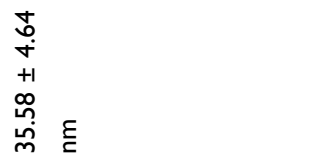 \\
\hline 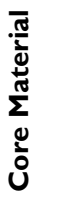 & 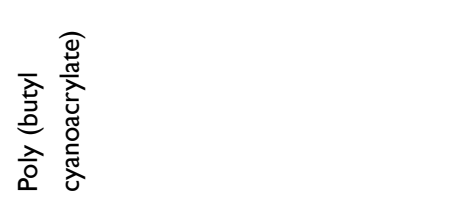 & 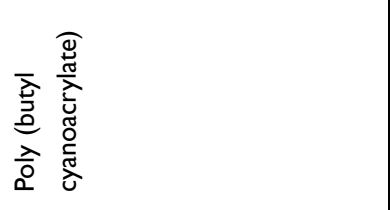 & 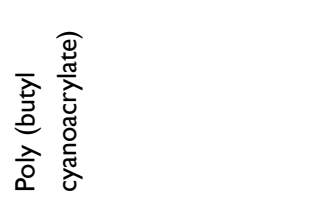 \\
\hline 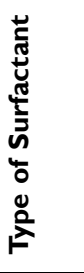 & 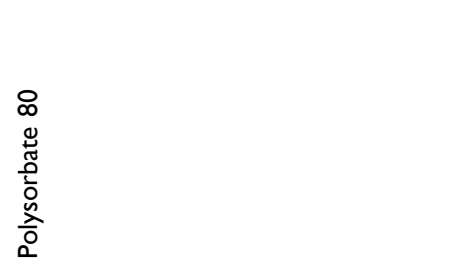 & 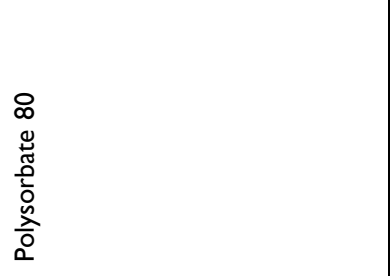 & 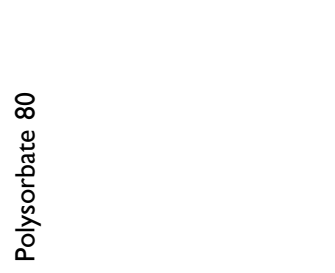 \\
\hline 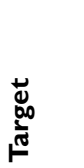 & 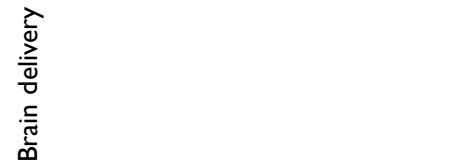 & 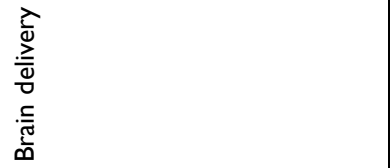 & 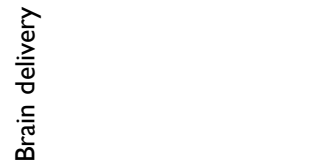 \\
\hline
\end{tabular}




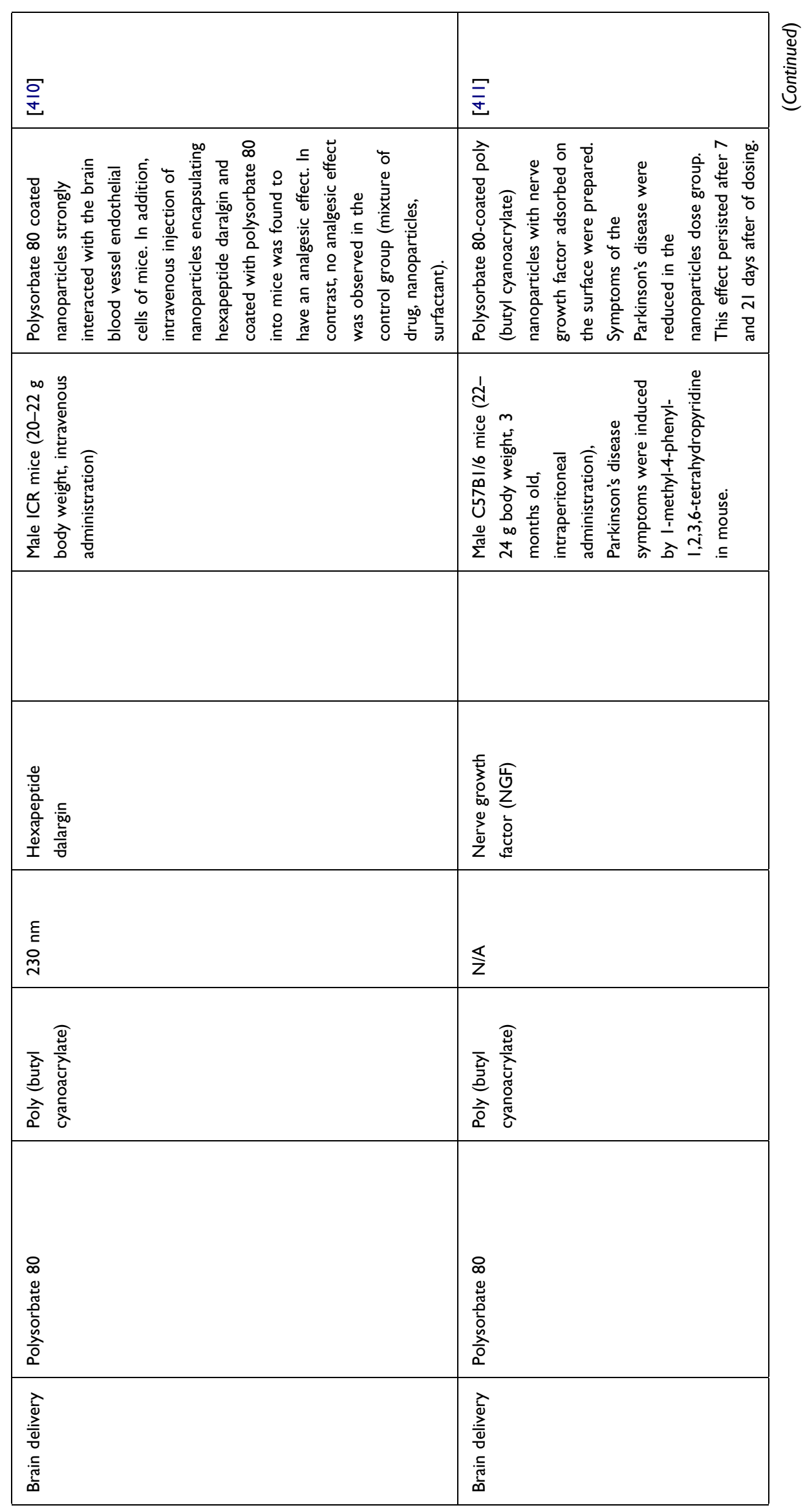




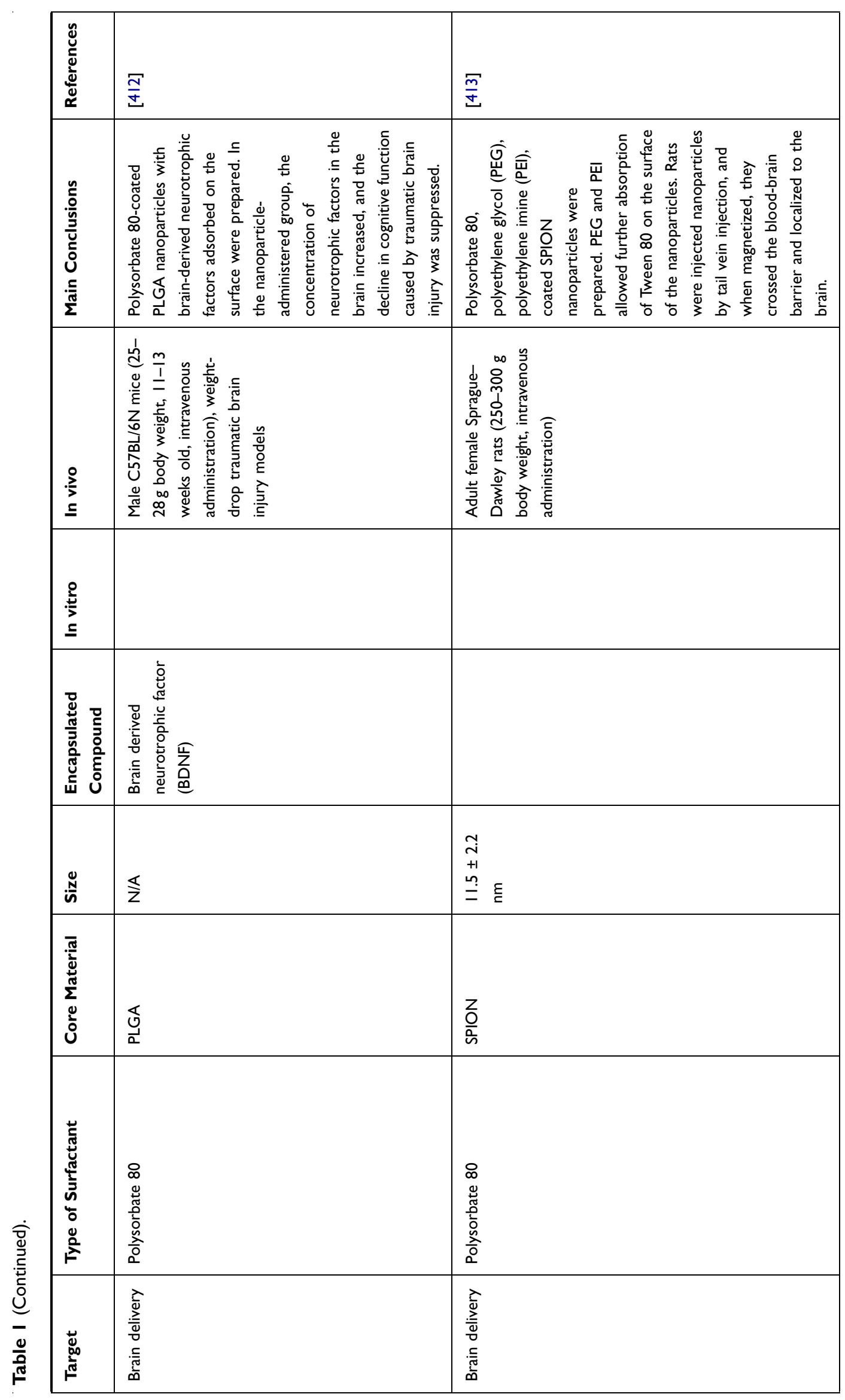




\begin{tabular}{|c|c|c|}
\hline$\frac{F}{\dot{E}}$ & 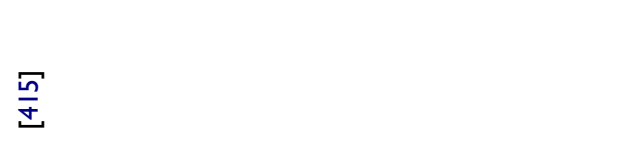 & $\frac{\Xi}{\Phi}$ \\
\hline 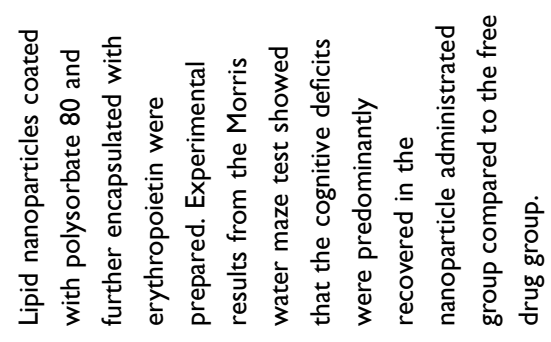 & 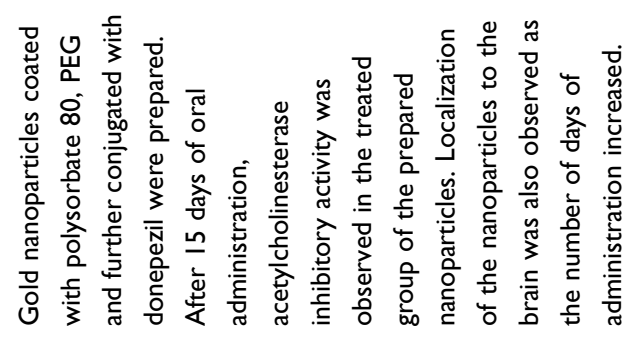 & 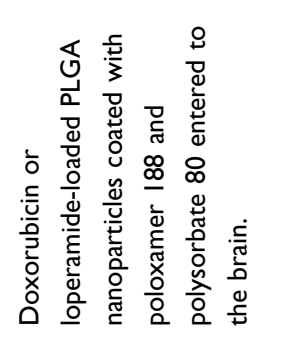 \\
\hline 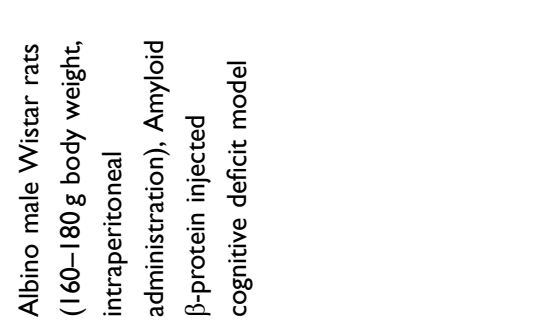 & 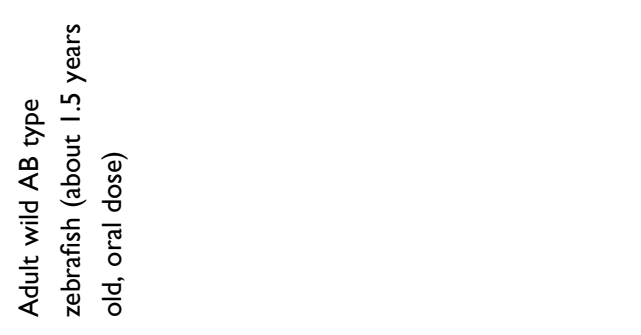 & 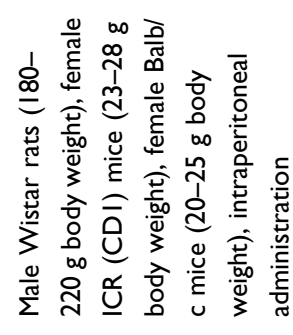 \\
\hline 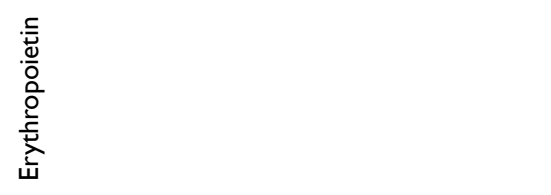 & $\begin{array}{l}\overline{\bar{N}} \\
\overline{0} \\
\overline{0} \\
\overline{0}\end{array}$ & 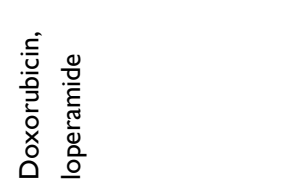 \\
\hline 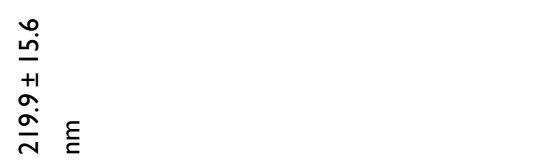 & $\begin{array}{l}\varepsilon \\
\vdots \\
0 \\
0 \\
1 \\
0\end{array}$ & 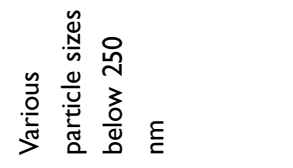 \\
\hline$\frac{\overline{0}}{\bar{a}}$ & $\frac{7}{0}$ & 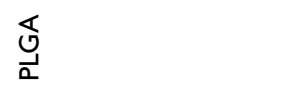 \\
\hline 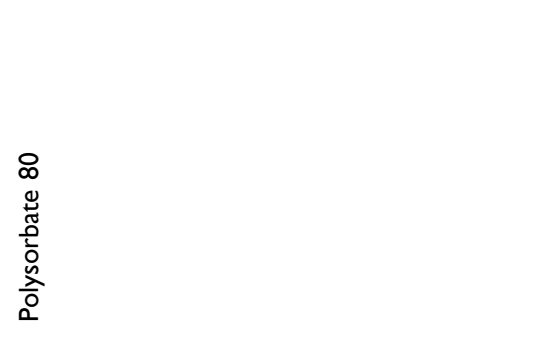 & 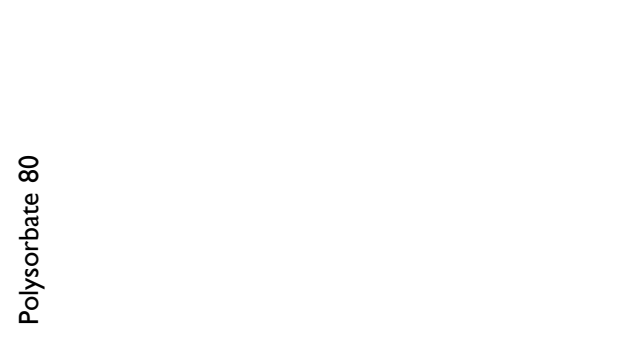 & 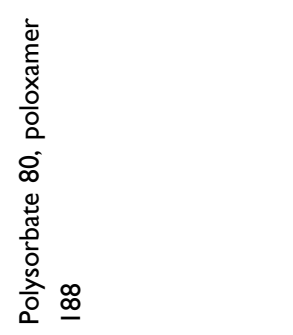 \\
\hline 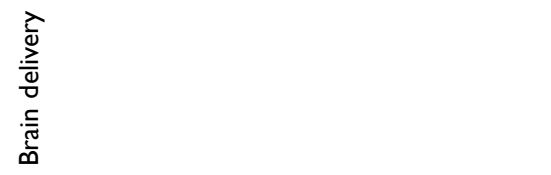 & 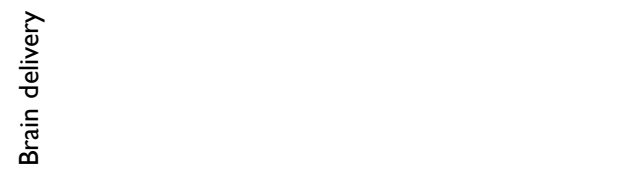 & 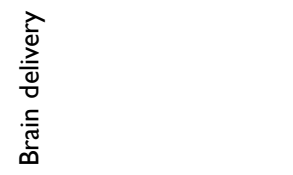 \\
\hline
\end{tabular}




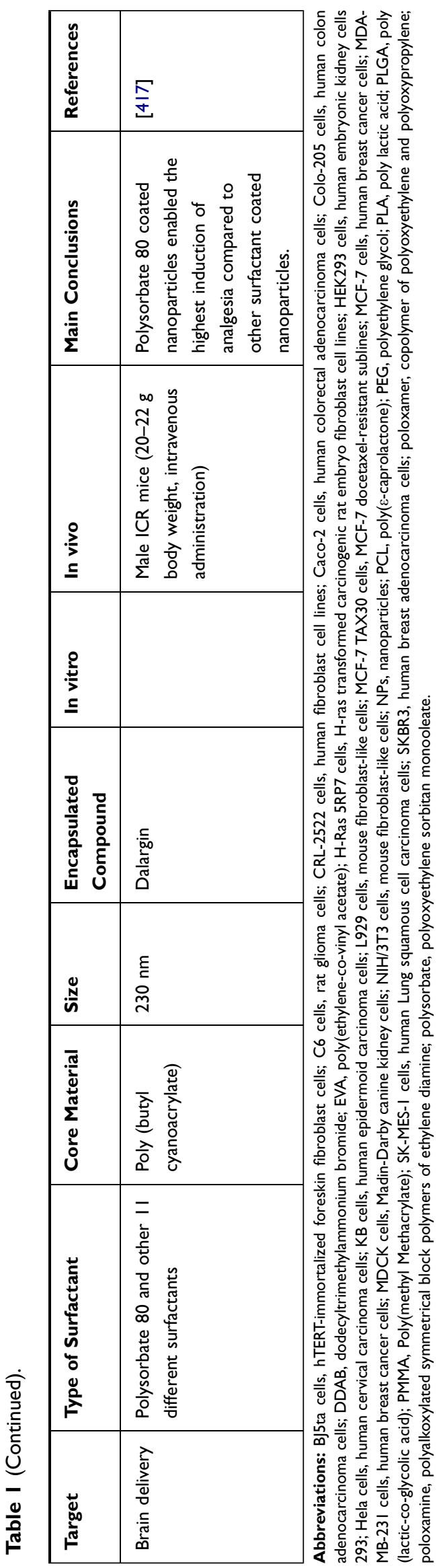

microscopy and transmission electron microscopy (TEM). ${ }^{94}$ The results showed that in the TC group, the precipitated particles showed acorn shaped (PBOB and PLGA individuals precipitated independently) morphology, while in the PVA group, both acorn and core-shell shaped morphologies were precipitated. As the reason for the difference in particle deposition morphology, they found that PVA forms a stable phase at the dichloromethane-water interface but has properties that prevent PLGA molecules from adsorbing to the interface, while TC does not allow other chemical species to adsorb at the interface. On the other hand, in the SC group, a mixed interface of PLGA molecules and surfactant was formed during particle formation, and particles with a core-shell shaped morphology were stably deposited in the system. Therefore, they concluded that when preparing particles by solvent evaporation technique, core-shell morphology was obtained if PLGA molecules could be adsorbed on the mixed interface, otherwise acorn shaped morphology was obtained. The coexistence of different surfactants may be useful in the formation of particles. Ramirez et al reported that when PLGA nanoparticles were prepared by the solvent evaporation technique, the presence of not only PVA but also other surfactant (SDS) leads to steric stabilization in the systems, resulting in the precipitation of PLGA nanoparticles with a smaller particle size than those prepared by PVA alone. ${ }^{95}$ Such findings suggest that surfactants play a critical role in the preparation of nanoparticles. The prepared nanoparticles were administered in vivo after their stability, interactions with proteins and cells have been thoroughly investigated in vitro. ${ }^{96}$

\section{Surface Charge and Protein Adsorption}

The charge on the surface of the nanoparticles has an important influence on their intracellular localization. Compared to anionic and nonionic charged nanoparticles, cationic charged nanoparticles exhibit higher cellular uptake due to their enhanced adhesion to the surface of negatively charged cells by electrostatic attraction. ${ }^{97-99}$ It has also been reported that cationic charged nanoparticles incorporated into cells have the ability of endosomal escape. Lipid nanoparticles composed of ionized amine lipids with a pKa of 6-7 and tertiary amines have an electrically neutral surface charge in the blood ( $\mathrm{pH}$ 7.4) but become cationic in the endosomal environment $(\mathrm{pH}<6.5)$ after they are taken up into the cell. As a result, cationic charged nanoparticles fuse with the negatively charged endosomal membrane and release encapsulated drugs into the cytoplasm. ${ }^{100-102}$ By this mechanism, 
cationic surfactants such as cetyltrimethylammonium bromide (CTAB) and didodecyldimethylammonium bromide (DDAB) are used to provide a positive charge to the surface of nanoparticles (Figure 1). Fay et al prepared cationic charged surfactant-coated nanoparticles (PLGA nanoparticles encapsulating plasmid DNA covered with cationic surfactant $[\mathrm{DDAB}])$ and assessed their transfection efficiency into murine macrophage (RAW 264.7) cells, and observed an increase in cellular uptake and endosomal escape; transfection was achieved with a one thousandth amount of plasmid DNA compared to that of commonly used transfection reagent Lipofectamine ${ }^{\circledR} \cdot{ }^{103}$ In addition, cationic charged nanoparticles showed a stronger immune response than anionic charged and nonionic nanoparticles, which have attracted attention in recent years for the development of vaccines and application in the field of immunotheraphy. ${ }^{104,105} \mathrm{Kedmi}$ et al prepared cationic charged surfactant-coated nanoparticles (small interfering RNA [siRNA] encapsulated in solid lipid nanoparticles coated with a cationic surfactant [1, 2-dioleoyl3-trimethylammonium-propane (DOTAP)]) and observed the activation of the innate immune system in C57BL/6 mice. ${ }^{106}$ The results showed a 10 - to 75 - fold higher induction of type 1 helper (Th1) cytokine expression than the control particles (weakly anionic charged). However, cationic charged nanoparticles are more likely to disrupt cell membrane integrity and cause damage to mitochondria and lysosomes than anionic charged and nonionic nanoparticles, which raises concerns about their side effects. ${ }^{107}$ It has also been reported that the surface of cationic nanoparticles is prone to non-specific adsorption of albumin and alpha-1Bglycoprotein. ${ }^{108,109}$ Furthermore, as mentioned in section "Overview of Surfactant" of this review, the cationic surfactant itself has potential toxicity; approaches to avoid this toxicity have been reported, for example, Gossmann et al observed reduced side effects when the surface of PLGA nanoparticles coexisted with nonionic polymers (polyethylene glycol [PEG]) and cationic surfactant (DDAB) in vitro.110 The RES is actively involved in the phagocytosis of macrophages in the spleen, bone marrow, and liver. ${ }^{111-113}$ Nanoparticles administered into the bloodstream bind to proteins and antigens called opsonin, forming a corona (a complex of nanoparticles, proteins, and antigens), which is taken up by macrophages. This phenomenon is called "opsonization", and the process involves apolipoprotein, albumin, fibrinogen, immunoglobulins, and complement components. ${ }^{114}$ The opsonized nanoparticles interact with receptors on the surface of macrophages and are transported to phagosomes and fused with lysosomes for degradation or elimination from the body. ${ }^{111}$ It has been reported that PLGA nanoparticles, which are not coated with any surfactant, are opsonized by non-specific adsorption of plasma proteins on their surface, which leads to their degradation in the body (Figure 4A). ${ }^{115}$ Moreover, targeting ligands present on the surface of the nanoparticles are masked by opsonization, which reduces their targeting ability. Salvati et al prepared silica nanoparticles modified with transferrin on its surface as a targeting ligand for receptor (transferrin receptor) on cancer cells and reported that the opsonized form of these nanoparticles lost their targeting ability. ${ }^{116}$ Hence, it is critical to avoid opsonization for effective targeting ability of nanoparticles in vivo and to reach the target site of action. Furthermore, it has been discovered that the nanoparticles coated with nonionic surfactants, such as poloxamers, avoid opsonization and predation by macrophages (this phenomenon is also called as "stealth effect") (Figure 4B). Currently, PEG modification of nanoparticles is the most widely used method to impart stealth effect to nanoparticles, but the continuous administration of PEG-modified nanoparticles has raised concerns about the accelerated blood clearance $(\mathrm{ABC})$ phenomenon (an immune response-induced mechanism to remove PEG-modified nanoparticles from the body).117-119 $\mathrm{Su}$ et al synthesized PEGylated surfactant by conjugation of surfactant (cholesteryl methyl amide) to PEG. ${ }^{120}$ They have reported that nanoemulsions composed of PEGylated surfactant showed weak ABC phenomenon in male Wistar rats. In the future, the properties of surfactants will be pursued more deeply, and surfactants that can modify the function of PEG and weaken the ABC phenomenon will be found. Jain et al prepared iron-encapsulated PLGA nanoparticles by optimizing the surface modification with a nonionic surfactant (poloxamer 188) using adsorption isotherm models (Langmuir, BET, Freundlich, Henderson, and Halsey models). ${ }^{121}$ The uptake of these surfactant-coated nanoparticles into murine macrophage (RAW 264.7) cells was compared with that of bare nanoparticles. The results revealed no cellular uptake of surfactant-coated nanoparticles after one hour of incubation. Liao et al prepared surfactant-coated nanoparticles composed of retinoic hydroxamic acid coated with nonionic surfactants (poloxamer 184 and 188) and observed their anticancer activity in subcutaneous melanoma (A375) mouse model. ${ }^{122}$ They reported that surfactantcoated nanoparticles exhibited a stealth effect in the body of mice, and showed enhanced anticancer activity due to increased accumulation in cancer cells and decreased accumulation in the liver during the $16 \mathrm{~h}$ observation period, compared to bare nanoparticles. The principle mechanism 


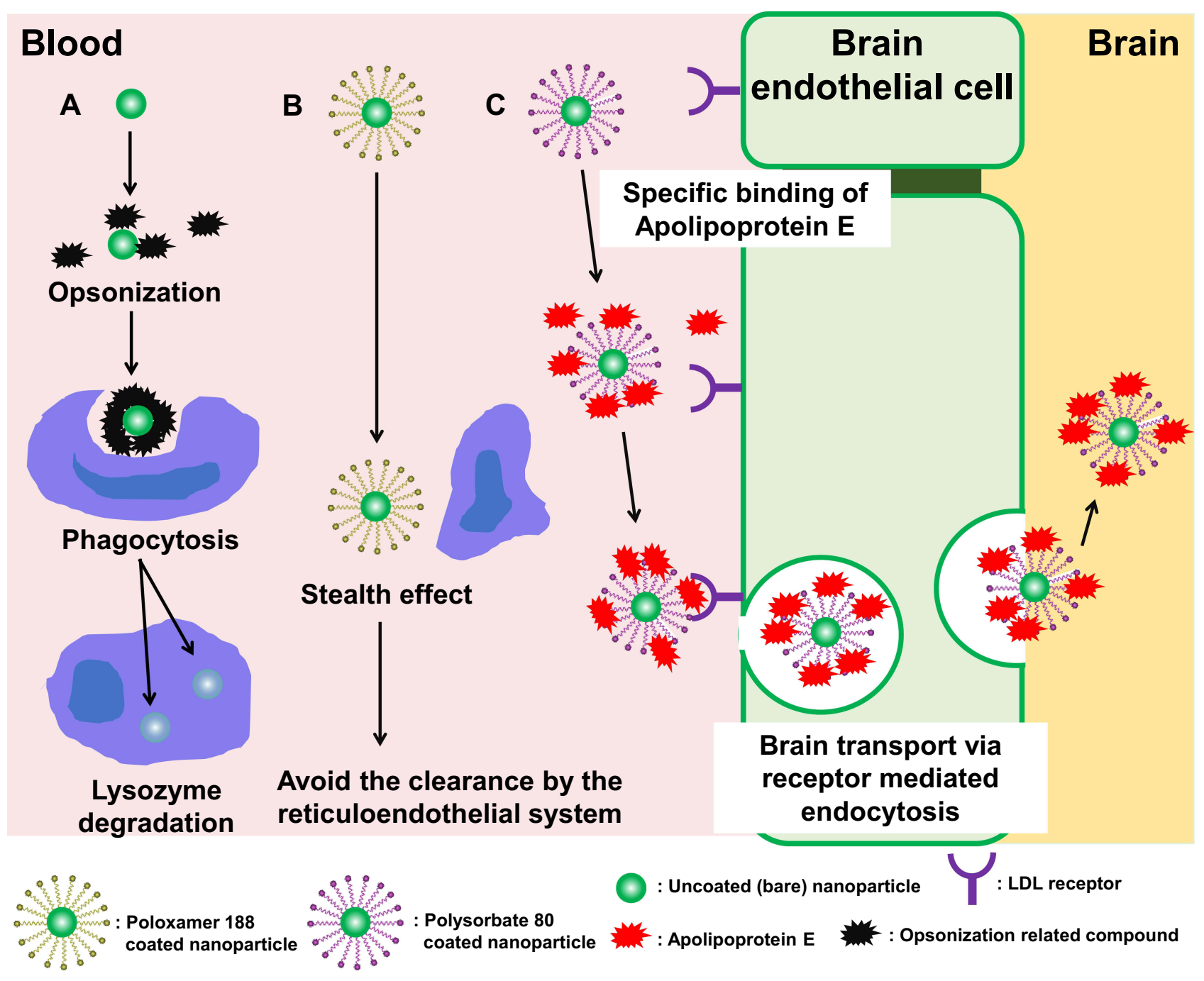

Figure 4 Behavior and fate of surfactant-coated nanoparticles in the blood stream.

Notes: (A) Bare nanoparticles. (B) Poloxamer 188 coated nanoparticles. (C) Polysorbate 80 coated nanoparticles.

by which poloxamer-coated nanoparticles exerted a stealth effect is due to the influence of PEG and polyoxyethylene oxide (PEO) moieties in chemical structure of poloxamer.${ }^{123,124}$ Surfactants and polymers with PEG, PEO, and polypropylene oxide (PPO) moieties are known to inhibit the adsorption of opsonins by building a hydrophilic barrier on the surface of the nanoparticles and by free movement and steric hindrance due to the construction of a polymer brush structure. ${ }^{125-128}$ This stealth effect has been observed not only with poloxamers but also with other nonionic surfactants having PEG and/or PEO moieties. For example, Zhao et al prepared surfactant-coated nanoparticles (gold nanoparticles covered with a nonionic surfactant [polysorbate 80]) and reported that adsorption of opsonization-related substances (bovine serum albumin [BSA], fibrinogen, $\gamma$-globulins, immunoglobulin $\mathrm{G}[\mathrm{IgG}]$, and lysozyme) on surfactant- coated nanoparticles in phosphate buffer was inhibited, and no aggregation was observed for 24 hours. ${ }^{129}$ On the other hand, there is a theory of the mechanism of the stealth effect of nonionic surfactants related to change in the conformation of the opsonins attached to the surfactant. Torcello-Gómez et al prepared surfactant-coated nanoparticles (polystyrene nanoparticles covered with a nonionic surfactant [poloxamer 188]) and confirmed their adhesive dynamics with IgG, which is a typical example of opsonin. ${ }^{130}$ They reported that the adhesion of IgG on the surface of surfactant-coated nanoparticles was only slightly inhibited compared to bare nanoparticles, and $80 \%$ of the surface area was covered by IgG. However, the conformation of IgG that adhered to nonionic surfactants changed, suggesting that the suppression of opsonization is not due to adhesion but due to conformational changes in IgG. Although imparting the stealth 
effect to the nanoparticles by using nonionic surfactants is easy and bears low cost, but the potential problems need to be solved. One of such problem is the possibility of detachment of surfactants from the nanoparticles and causes unexpected side effects in vivo; the physiological effects of the autoxidized and hydroxylated products of nonionic surfactants, and their complement activation in vivo are largely unknown. ${ }^{76,131-133}$ One way to address these concerns might be to optimize the interaction between the encapsulated drug and the materials of the nanoparticles. Gagliardi et al compared zein and PLGA as suitable materials for the preparation of nanoparticles encapsulating lipophilic flavonoid (rutin). ${ }^{134}$ The results showed that the interaction between rutin and zein exhibited longer drug release kinetics in the zein group compared to the PLGA group, and this effect was most effectively demonstrated when sodium deoxycholate monohydrate (SD) was used in the preparation of nanoparticles. In the future, more useful surfactant-coated nanoparticles will be developed by further optimizing the compatibility of the encapsulated drug, nanoparticle material, and coating surfactant.

\section{Active and Passive Targeting}

In addition to the adsorption and surface modification of the nanoparticles, the particle size is a major factor governing the behavior of nanomedicine. It is generally accepted that the desired particle size for solid particles administered as drugs for circulation in the bloodstream is 10-200 nm (Figure 5A). ${ }^{135}$ Particles smaller than $100 \mathrm{~nm}$ in size are known to avoid phagocytosis by the RES and have been reported to circulate in the bloodstream for a relatively long time. ${ }^{136-138}$ On the other hand, since the diameter of capillaries in the body is 3-9 $\mu \mathrm{m}$, particles larger than that size can clog capillaries and unintentionally accumulate in organs with large surface areas of capillaries, such as the lungs. ${ }^{139}$ Kutscher et al found that particles with a size of $6 \mu \mathrm{m}$ or larger accumulated in the lungs for more than a week when polystyrene microparticles of different particle sizes $(2,3,6$, and $10 \mu \mathrm{m})$ were administered intravenously to rats. ${ }^{140}$ In addition, particles larger than $400 \mathrm{~nm}$ in size were captured by splenic filtration, and then removed and degraded by red pulp macrophages. ${ }^{141}$ Conversely, it has been also reported

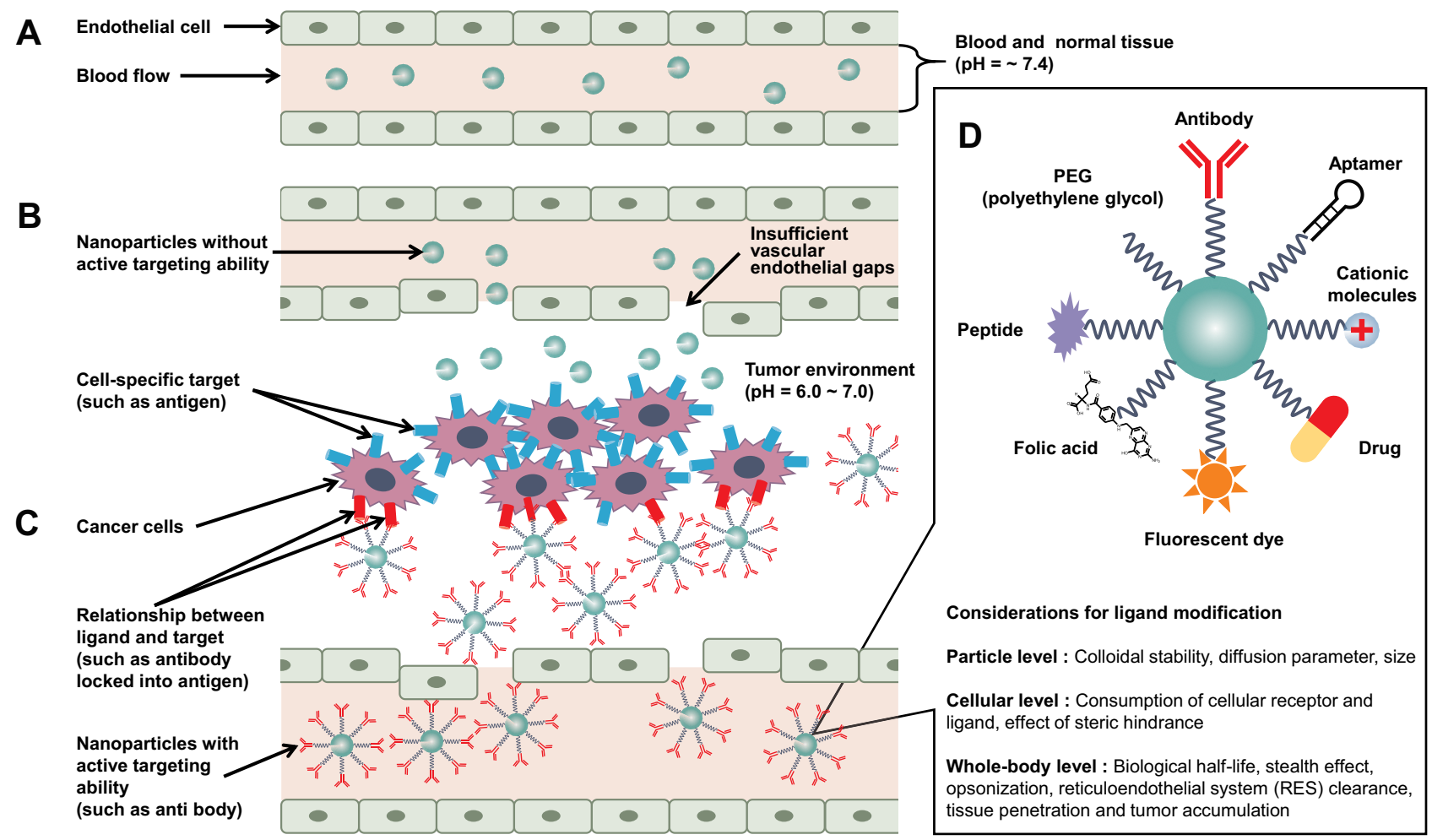

Figure 5 Active and passive targeting of nanoparticles to the cancer cells.

Notes: (A) Normal vasculature. (B) Passive targeting in tumor vasculature. (C) Active targeting in tumor vasculature. (D) Types of active targeting ligands for nanoparticles and its considerations for optimization of their efficacy. Notes: (B, C) Adapted from Tran S, DeGiovanni P, Piel B, et al. Cancer nanomedicine: a review of recent success in drug delivery. Clin Transl Med. 2017;6(1):44. doi:10.1 186/s40169-017-0175-0. ${ }^{153}$ (D) Adapted from Advanced Drug Delivery Reviews, I43, Alkilany AM, Zhu L, Weller H, et al, Ligand density on nanoparticles: a parameter with critical impact on nanomedicine, 22-36, Copyright 2019, with permission from Elsevier. $^{158}$ 
that too small particle size can make it difficult to circulate in the bloodstream. Particles smaller than $15 \mathrm{~nm}$ are filtered out of the bloodstream by the kidneys and removed from the bloodstream. ${ }^{142}$ As the average effective pore size of normal vascular endothelial cell is approximately $5 \mathrm{~nm}$, some reports suggest that particles with a size smaller than $5 \mathrm{~nm}$ leak out of vascular endothelial cells and accumulate at unintentional sites, causing them to disappear from the bloodstream in a short time. ${ }^{143}$ Particle size is also important in the development of cancer-targeting drug delivery systems. One of the most recognized cancer-targeting effects is the enhanced permeability and retention (EPR) effect, which was reported by Matsumura and Maeda in $1986 .{ }^{144}$ The following two phenomena are collectively referred to as the EPR effect: (1) the presence of gaps in the new blood vessels around the tumor due to an incomplete vascular endothelial system, which allows nanoparticles to pass through the vessel wall and accumulate in the tissue; and (2) long-term accumulation of nanoparticles in the tumor tissue due to insufficient intratumoral exclusion system consisting of immature lymphoid tissue in cancer cells than in normal cells (Figure 5B). The EPR effect is referred to as "passive targeting" because it does not require surface modification with targeting ligand. The EPR effect is reported to be exhibited by particles having size of $100-400 \mathrm{~nm} .{ }^{145}$ Based on this mechanism, a number of studies on cancer targeting chemotherapy using nanoparticles with a particle size of $400 \mathrm{~nm}$ or less have been reported to date. ${ }^{146-150}$ On the other hand, many researchers believe that EPR effect alone is not sufficient to achieve cancer-targeting therapeutic effect of nanoparticles, and further enhancement is required, as observed in some gastric and pancreatic cancers. ${ }^{151}$ Sindhwani et al reported in 2020 that the accumulation of nanoparticles in solid tumors is dominated via trans-endothelial pathways than by EPR effects, which has attracted much attention. ${ }^{152}$ In addition to the EPR effect, "active targeting" has been widely attempted to further enhance the therapeutic effects of nanoparticles. Active targeting refers to the modification of nanoparticles with targeting ligands (antibodies, aptamers, carbohydrates, macromolecules, proteins, and small molecules) for cancer cell-specific targets (antigens, lipid components, receptors, or proteins on the cell membrane). The drug encapsulated in the nanoparticles modified with the targeting ligand accumulates around the tumor tissue by the EPR effect (passive targeting) and is delivered and accumulated at the target site of cancer cells through response, affinity, and binding by the molecular site, shape, and stimulation (such as $\mathrm{pH}$, temperature, and ultrasound) (Figure 5C and D). ${ }^{153,154}$ Tumors with a volume of less than $100 \mathrm{~mm}^{3}$ have insufficient vascular endothelial gaps and are recognized as less effective for drug accumulation via EPR effect, while active targeting is regarded as effective in treating such small tumors and other diseases. ${ }^{155}$ Acharya et al prepared rapamycin-encapsulated PLGA nanoparticles. ${ }^{156}$ They reported that when their surface was modified with epidermal growth factor receptor monoclonal antibodies (EGFR mAb) (passive targeting + active targeting), their uptake into malignant breast cancer (MCF-7) cells was 13-fold higher than that of bare (passive targeting only) nanoparticles. Poom et al prepared PEG nanomicelles containing anticancer drug (paclitaxel) and reported that the accumulation of paclitaxel in rat tumor tissue decreased to $1 \% \mathrm{ID} / \mathrm{g}$ of tissue after 3 days when the PEG nanomicelles were administered (passive targeting only), whereas the drug accumulation of more than $5 \% \mathrm{ID} / \mathrm{g}$ of tissue was maintained even after 5 days when the PEG nanomicelles modified with folate ligands were administered (passive targeting + active targeting). ${ }^{157}$ However, excessive surface modification of nanoparticles with targeting ligands is thought to result in poor targeting to cancer cells due to the following factors: (1) decrease in the stealth effect due to the reduced surface exposure of molecular sites such as PEG, PEO, and PPO, (2) decrease in the EPR effect with the increase in particle size, (3) reduced diffusion of nanoparticles in cancer tissue, (4) decrease in the ability to bind to cancer cell-specific targets due to steric hindrance between targeting ligands, and (5) a decrease in the number of particles taken up by increasing the receptor occupancy per particle (Figure 5D). ${ }^{158}$ Therefore, it is suggested to optimize the density of the targeting ligands for specific cancer cell targeting for maximum interaction between nanoparticles and target cells. Recently, several nanomedicine products based on nanoparticles have been approved by the Food and Drug Administration (FDA). ${ }^{159,160}$ Although the field of research on nanoparticle-based drug delivery systems is developing rapidly, there are many concerns that need to be considered in the future, especially when nanoparticles are not distributed within the tumor microenvironment depending on the condition of cancer, ${ }^{161}$ expression of surface receptors varies depending on the diversity of cancer (for example, active targeting not working well for cancer stem cells), ${ }^{162}$ and acquisition of drug resistance in cancer. ${ }^{163}$ 


\section{Brain Targeting}

Even if a substance is proven effective in treating brain tumors, neurodegenerative diseases and central nervous system diseases, to the most important challenge is to deliver it to the brain. Effective therapeutic antibodies are being developed to target brain diseases, however, brain delivery approach for these antibodies while maintaining their shape has yet to be developed. ${ }^{164}$ Nanotechnology is potentially used to protect encapsulated substances. Establishing technologies for transporting nanoparticles to the brain is one of the greatest obstacles in the field of nanomedicine. The major obstacle is the presence of the blood-brain barrier (BBB), which exists between the central nervous system and the blood, and greatly restricts the transport of substances to the brain. Substances circulating in the bloodstream can only pass through the BBB if these are (1) hydrophobic molecules of weight below $450 \mathrm{Da}$ or (2) transported via endogenous transporters present in the BBB. ${ }^{165,166}$ Therefore, regardless of the type of material used to prepare nanoparticles; it is difficult for them to reach the brain by simply injecting them intravenously in their original state. On the other hand, it has been reported that nonionic surfactant (for example, polysorbate 80)-coated nanoparticles with active targeting function could reach the brain; although the detailed mechanism of transport of nanoparticles into the brain by modification with polysorbate 80 is still unclear. The current prevailing theory is that apolipoprotein adsorption at the polysorbate 80 site of surfactant-coated nanoparticles circulating in the bloodstream that crosses the BBB through receptor-mediated transcytosis (Figure 4C). ${ }^{167,168}$ The use of nonionic surfactants such as polysorbate 80 may also help nanoparticles to accumulate in the brain for a long time due to their inhibitory effect on P-glycoprotein (Pgp/ABCB1, a mechanism of foreign body efflux in the brain). ${ }^{169,170}$ Other substances that use this mechanism of brain transport are poly (butyl cyanoacrylate) (PBCA) and PLGA. ${ }^{171}$ Wilson et al prepared surfactant-coated nanoparticles (rivastigmine-encapsulated PBCA nanoparticles coated with polysorbate 80) and quantitatively evaluated their transport to the brain. ${ }^{172}$ They administered surfactant-coated nanoparticles to a group of rats via tail vein injection and reported a fourfold increase in the concentration of rivastigmine in the brain one hour after administration compared to the group receiving free drug. Tahara et al prepared surfactant-coated nanoparticles (coumarin-6 encapsulated PLGA nanoparticles coated with polysorbate 80 ) and quantitatively evaluated their transport to the brain. ${ }^{173}$ They reported that the surfactant-coated nanoparticles administered to a group of rats via tail vein injection showed a two-fold increase in the concentration of coumarin- 6 in the brain one hour after administration, compared to a group of rats being administered bare nanoparticles (without surfactant coating). Furthermore, they reported that the increased transport to the brain was specific only to the nanoparticles coated with polysorbate 80 , and similar result was not demonstrated by chitosan or other nonionic surfactants (poloxamer 188). The transport of surfactantcoated nanoparticles into the brain has also been studied using surfactants other than polysorbate 80 , such as polyoxyethylene esters of 12-hydroxystearic acid (Solutol ${ }^{\mathbb{B}}$ HS15, BASF corporation, Ludwigshafen, Germany) and D-alpha-tocopherol polyethylene glycol 1000 succinate, however, the mechanism of their transport is not clear.174,175 Many studies on brain transport of surfactant-coated nanoparticles have reported only blood concentration and brain accumulation, but it is also important to evaluate the drug accumulation in other non-specific organs. Miyazawa et al prepared surfactant-coated nanoparticles (PLGA nanoparticles encapsulated with $\beta$-carotene and coated with polysorbate 80 ), and quantitatively evaluated their accumulation in the brain and other organs in rats via tail vein administration. ${ }^{176}$ They reported that the surfactantcoated nanoparticles administered group showed higher drug accumulation in the lungs (350-fold higher concentration compared to the group of bare nanoparticles) than in the brain after one hour of administration. A similar phenomenon has been reported in the study by Tröster et al, who prepared polymethyl methacrylate resin nanoparticles coated with various nonionic surfactants (polysorbates [20,60, and 80], poloxamers [184, 188, 338, 407 , and 908], and polyoxyethylene lauryl ether [Brij 35]) and administered them to rats via tail vein to compare their accumulation in organs over time. ${ }^{177}$ In their report, compared to the bare nanoparticles, the particles coated with polysorbate 80 had an approximately 11-fold increase in accumulation in the lungs and a nine-fold increase in accumulation in the brain after 30 min of administration. They also reported that approximately half of the particles that had accumulated in the lungs migrated to the liver two hours after administration. Therefore, increasing drug concentrations at the target site of action can enhance the desired therapeutic effect, but significant toxicity may 
also occur because of the increased drug accumulation in non-specific organs.

While polysorbates and poloxamers have been reported to perform such useful functions, there are concerns about their side effects that cause cell membrane damage and cytotoxicity. ${ }^{178}$ Recently, potential surfactants other than poloxamer and polysorbate have been discovered for brain targeting. For example, Jeong et al prepared surfactantcoated nanoparticles (PLGA nanoparticles encapsulated with recombinant human erythropoietin [rhEPO] and coated with sodium cholate or polysorbate 80 ) and evaluated their cellular uptake (human neuroblastoma [SHSY5Y] cells) and evaluated inhibition rate of glutamateinduced neurotoxicity. ${ }^{179}$ The results showed that the sodium cholate-coated nanoparticle group was taken up by SH-SY5Y cells and further reduced glutamate-induced neurotoxicity with less toxicity than the polysorbate 80 coated group. They also examined the efficacy of these nanoparticles in vivo experimental stroke model mice and reported that the symptoms were reduced. ${ }^{180}$ It is expected that a variety of surfactants targeting the brain will be developed in the future. In recent years, the importance of "inter-organ communication," which considers treatment based on the interaction of the drug with entire body's organs, and not just the individual organ has been recognized; this concept will also be essential for the development of surfactant-coated nanoparticles. ${ }^{181}$

\section{Surfactant-Coated Inorganic Nanoparticles in Nanomedicine}

Various types of inorganic materials have been used in nanomedicine. In this section, nanoparticles composed of gold and silicon, which have been specially studied, and the applications of inorganic nanoparticles in quantum dots and magnetic resonance imaging (MRI) are mainly discussed (Figure 2B).

\section{Gold Nanoparticles}

Gold nanoparticles are one of the most widely used inorganic nanoparticles in nanomedicine due to their ease of preparation, high dispersion, low toxicity, and stability (Figure 2B). And several studies of surfactant-coated gold nanoparticles have been conducted (Table 1). Gold nanoparticles have a long history of research, and the first report on the preparation method by Michael Faraday in 1857 used chloroauric acid solution. ${ }^{182}$ A typical method for the preparation of gold nanoparticles is the Turkevich method (method of reducing $\mathrm{Au}^{3+}$ and $\mathrm{Au}^{+}$to $\mathrm{Au}^{0}$, which is the electrical state of nanoparticles, using trisodium citrate), which was reported in 1951 and is still widely used today ${ }^{183,184}$ Subsequently, various preparation methods based on chemical reduction of gold ions in the solution have been developed, and gold nanostructures of various shapes (such as nanobowls, nanocages, nanocubes, nanopyramids, nanorods, nanospheres, nanoshells, nanostars, and nanowires) have become available and have been studied and developed into nanomedicine. ${ }^{185-187}$ Nanoparticles composed of transition elements such as gold have an optical property called surface plasmon resonance (SPR). SPR is a phenomenon in which plasma oscillation on the surface of a transition element generates an electric field around it, and this electric field resonates with light, resulting in strong absorption and scattering of light at a specific wavelength. This property has been used to study the enhanced Raman imaging of transition-element nanoparticles. The absorption maximum of SPR depends on the type of transition element present in the nanoparticles. ${ }^{188}$ Since enhanced Raman imaging directly detects molecular vibrations, the dynamics of biomolecules in living cells can be observed label-free. This advantage has led to the use of gold nanoparticles for molecular imaging of living cells and elucidation of the functions of biomolecules. ${ }^{189,190}$ Furthermore, since the extinction coefficient of gold nanoparticles is more than 1000 times higher than that of organic dyes, photothermal treatment using the photothermic properties of SPR is also being investigated. ${ }^{191,192}$ Furthermore, the photothermic properties of gold nanoparticles leads to a thermoelastic expansion that is converted into a photoacoustic wave with increase in temperature. This property has also been used in research on photoacoustic imaging. ${ }^{193,194}$ The synthesis of gold nanoparticles is not limited to chemical approaches, but the synthesis of gold nanoparticles using living organisms such as algae, bacteria, and plants (biobased method) is a topic. In general, the bio-based method is regarded as having the advantages of not using harmful chemicals in the synthesis process and being low cost. ${ }^{195}$ In addition to the advantages of the bio-based method described above, the use of cultured cell lines to synthesize gold nanoparticles may be less toxic than bio-based method that use bacteria composed of substances that may cause inflammation (surface of synthesized nanoparticle by using bacteria is coated by substances [lipopolysaccharides and endotoxins] derived from bacteria). It is also expected to enable in-situ synthesis of gold nanoparticles inside the tumor for photochemotherapy. Singh et al 
confirmed in detail the mechanism by which nanostructures of gold nanoparticles are formed by human breast cancer (MCF7) cells from the medium containing gold (III) chloride hydrate. ${ }^{196}$ They found that the cells were stressed by the presence of low doses of gold ions, resulting in a reversible state of cellular senescence. For this stress, under serum containing medium (Dulbecco's Modified Eagles Medium (DMEM) supplemented with $10 \%$ fetal bovine serum (FBS)), the cells became unstressed state and gold nanoparticles with rounded facets (single and agglomerated) were deposited. On the other hand, in serum-free medium, the cells were in a stressed state and released various secretions outside the cells. Among these secretions, the presence of gold-binding proteins significantly affected the crystal growth of gold nanoparticles, and gold nanoparticles with sharp facets (triangular and hexagonal) were deposited. They further synthesized gold nanoribbons by incubating human breast cancer (MCF7) cells with gold (III) chloride hydrate. ${ }^{197}$ They confirmed that the gold nanoribbons synthesized by this method had anisotropic and high aspect ratio and showed more efficient energy conversion effect than spherical gold nanoparticles or gold nanorods. The molecular dynamic simulation and supported with experimental photothermal therapy shown useful application of these nanomaterials into nanomedicine for promoting the growth of fibroblast, imaging agent, ${ }^{198}$ drug carrier with improved bioavailability in vitro ${ }^{199}$ and in vivo. ${ }^{200}$ Currently, there are no medical devices for imaging of gold nanoparticles that can be used for clinical applications, and further development of this technology is warranted. On the other hand, some clinical uses of gold nanoparticles themselves include Aurimune ${ }^{\circledR}$ (CYT-6091; CytImmune Sciences, Rockville, MD, USA), which is designed to deliver tumor necrosis factor (TNF- $\alpha$ ) to tumor sites, and AuroShell ${ }^{\circledR}$ (Nanospectra Biosciences Inc., Houston, TX), which is designed to enhance the efficacy of near-infrared laser therapy; both of them have been developed as gold nanomedicine products and are in clinical trials. $^{201,202}$

\section{Silica Nanoparticles}

Silica nanoparticles are inorganic solids that are ubiquitously present in the human body (Figure 2B). ${ }^{203}$ Silanol groups on the surface of silica can be easily modified by targeting ligands such as small molecules, carbohydrates, antibodies, aptamers, proteins, and polymers. Therefore, fluorescent compoundencapsulated silica nanoparticle with active targeting function on its surface has been studied as bioimaging probes and photodynamic therapy for cancer treatment. ${ }^{204,205}$ The typical methods for the preparation of silica nanoparticles are the Stöber method, which includes adding acid or alkali to an alkoxysilane solution to progress hydrolysis and polycondensation reactions, and the reverse micelle method, which includes adding an alkaline solution and a surfactant in a hydrophobic organic solvent to form a reverse micelle, and then adding an alkoxysilane solution to proceed with the hydrolysis and polycondensation reactions. ${ }^{206,207}$ Attempts have also been made to reduce the toxicity of highly toxic nanoparticles, such as metals by using the properties of silica. Iqbal et al reported that coating the surface of superparamagnetic iron oxide nanoparticles (SPION), which are used as MRI contrast agents, with silica greatly reduced their cytotoxicity while maintaining their function as MRI contrast agents. ${ }^{208} \mathrm{On}$ the other hand, mesoporous silica nanoparticles composed of porous silica, are relatively new materials whose synthesis was reported by Yanagisawa et al and Mobil Research and Development Corporation in the early 1990s, respectively.209,210 Typical preparation method of mesoporous silica nanoparticles includes preparation of a porous structure by using a supramolecular structure containing surfactant as a template. The size and shape of the pores can be manipulated to some extent, depending on the preparation conditions. ${ }^{211}$ Mesoporous silica nanoparticles have the following characteristics: (1) pores of 2-30 $\mathrm{nm}$ can be prepared with uniform size and distribution, (2) a large pore volume of approximately $1 \mathrm{~cm}^{3} \mathrm{~g}^{-1}$ can be achieved, (3) they have a high density of silanol groups on their surfaces, and (4) they have a chemically stable silica framework; these characteristics are likely to be beneficial for their use in nanomedicine. ${ }^{212}$ Although there have been concerns that the chemical structure of silica tends to become unstable in water and humid environments and that the silanol groups on the silica surface induce hemolysis, but the mesoporous silica nanoparticles with a smaller contact area of silanol groups with red blood cells have been found to be less prone to hemolysis than other silica nanoparticles. ${ }^{213-215}$ Urata et al prepared an ethylene-bridged silsesquioxane framework containing mesoporous silica nanoparticles to stabilize the skeletal structure and to inhibit the exposure of silanol groups, resulting in decreased hemolysis from $10 \%$ to a few percent and further stabilized the skeletal structure compared to ordinary mesoporous silica nanoparticles in vitro. ${ }^{216}$ Doxorubicin and paclitaxel have been known to be synergistic due to their different anticancer mechanisms. However, it has been difficult to prepare a stable single nanoparticle containing both drugs because of their different physicochemical 
properties. To solve this problem, Yan et al reported the preparation of mesoporous silica nanoparticles with doxorubicin being present inside the pores, and a derivative of paclitaxel to the surface of the particles and further coated the surface with polystyrene sulfonate. ${ }^{217}$ The prepared nanoparticles successfully released both the drugs according to the $\mathrm{pH}$ and redox status of the cancer cells in vitro. Although research on silicabased nanomedicine continues to make great progress, one of the reasons why it has not yet reached clinical trials may be its potential toxicity, which has not been dispelled. While the toxicity of crystalline silica particles due to occupational exposure is widely recognized, the potential toxicity of silica is considered proportional to its crystallinity, and therefore it is believed that amorphous silica particles are low in toxicity. ${ }^{218}$ Currently, epidemiological studies have not yet reached to a clear conclusion, and the safety of silica-based materials needs to be further confirmed. ${ }^{219}$ In recent years, a field of nanomedicine called "nanotheranostics" has been developed for the diagnosis and treatment of diseases at the same time, and the development of bio-imaging probes is under intense investigation in this field. ${ }^{220-222}$ The fluorescence imaging technology using quantum dots, which enables to visualize the behavior of individual cells in vivo and to treat them at the same time, has been attracting attention (Figure 2B). ${ }^{223}$ Quantum dots, which are colloids of semiconducting nanoparticles approximately 2-50 $\mathrm{nm}$ in size, have fluorescent properties compared to fluorophores: negligible fluorescence photobleaching in response to the excitation light, a broader excitation spectrum, and a sharper emission peak. ${ }^{224,225}$ Quantum dots with large particle sizes have a small band gap and emit red light, while quantum dots with small particle sizes emit blue light owing to their quantum confinement effect. Therefore, the light absorption and fluorescence emission wavelengths are shifted to the shorter wavelengths with higher energy as the particle size decreases. Due to these fluorescent properties, quantum dots can be used with a single light source to simultaneously excite and visualize target cells labeled with various types of quantum dots. The use of quantum dots has enabled imaging that was difficult to achieve with fluorophores including cytometric imaging, ${ }^{226}$ lymph node mapping, 227,228 imaging of cancer stem cells, ${ }^{229}$ and imaging of circulating tumor cells. ${ }^{230}$ However, since quantum dots are composed of semiconductor materials, side effects of heavy metals and their residues (selenium, cadmium, and lead) in living organisms are a major concern. $^{231,232}$ The shape of the nanoparticles is also related to cytotoxicity as shown in a study by Yamamoto et al that dendritic titanium dioxide particles with dendritic shape have higher cytotoxicity than spindle and spherical shapes. ${ }^{233}$ If quantum dots are to be used clinically in the future, these potential risks will need to be eliminated in advance. Recently, research and development of quantum dots composed of less toxic carbon, silicon, and germanium has initiated. ${ }^{234-236}$ Shen et al prepared surfactant-coated nanoparticles (quantum dots composed of silicon covered with poloxamer 407) and determined whether they could be used for the imaging of mitochondria in human umbilical vein endothelial cells (HUVECs) using confocal microscopy. ${ }^{237}$ As a result, they reported that the MitoTracker ${ }^{\circledR}$ (commonly used fluorophore for fluorescent staining of mitochondria) faded in 80 seconds, whereas surfactant-coated nanoparticles accumulated in the mitochondria with low toxicity for further use in living cells, and also maintained nearly constant fluorescence intensity for $30 \mathrm{~min}$.

\section{Other Inorganic Nanoparticles}

Nanomedicine is also used in MRI, which is an important imaging technique in contemporary medicine. MRI is used clinically to observe signals of hydrogen ions $\left({ }^{1} \mathrm{H}\right)$ contained in water molecules $\left(\mathrm{H}_{2} \mathrm{O}\right)$, and adipose tissues $\left(\mathrm{CH}, \mathrm{CH}_{2}\right.$, and $\left.\mathrm{CH}_{3}\right)$. The magnetization of hydrogen ions in a static magnetic field is excited by the magnetic resonance (MR) phenomenon when irradiated with radiofrequency magnetic waves (radiofrequency $[R F]$ pulses). MRI detects the relaxation time for these excited hydrogen ions to return to the ground state. The $\mathrm{T} 1$ relaxation time of hydrogen ions is observed to be greatly shortened by the presence of surrounding transition elements $\left(\mathrm{Cr}^{2+}, \mathrm{Cr}^{3+}, \mathrm{Mn}^{2+}, \mathrm{Mn}^{3}\right.$ ${ }^{+}, \mathrm{Fe}^{2+}$, and $\left.\mathrm{Fe}^{3+}\right)$ and lanthanides $\left(\mathrm{Gd}^{3+}\right.$ and $\left.\mathrm{Dy}^{3+}\right)$, and MRI contrast agent containing nanoparticles composed of these elements have been studied (Figure 2B). ${ }^{238,239}$ For example, ferucarbotran Resovist $^{\circledR}$, Bayer Healthcare, Leverkusen, Germany), ferumoxides (Feridex ${ }^{\circledR}$, Bayer Healthcare, Leverkusen, Germany), erumoxtran-10 (Combidex ${ }^{\circledR}$, AMAG Pharma, Waltham, MA), and NC100150 (Clariscan ${ }^{\circledR}$, Nycomed, Zürich, Switzerland), which are composed of SPION, have been used in clinical practice as MRI contrast agent nanoparticles. ${ }^{240}$ On the other hand, metal ions in MRI contrast agent nanoparticles have the potential risk of disrupting the redox balance in vivo by reacting with hydrogen peroxide to produce reactive oxygen species (ROS), and oxidizing vitamins and proteins, which are antioxidants present in the body; this leads to the progression and development of the disease. ${ }^{241}$ The accumulation of inorganic nanoparticles in monocytes, 
macrophages, and tissues can also cause inflammation; nanoparticles such as silica, gold, silver, carbon, iron, zinc, and titanium, have been reported to induce the production of various proinflammatory cytokines (interleukin [IL]-1 $\beta$, IL-6, IL-12, IL-23, and TNF- $\alpha$ ), and ROS associated with classically activated (M1) macrophages. ${ }^{242}$ These pro-inflammatory cytokines increase the expression of P-glycoprotein in various organs in vivo, and prevent likely functions of the nanoparticles. ${ }^{243,244}$ Radomski et al also reported that carbon nanoparticles interact with platelets and vascular endothelial cells, resulting in localized inflammation in vivo. ${ }^{245}$ Further studies are needed to avoid the potential health risks associated with such inorganic nanoparticles.

\section{Other Nanostructures in Nanomedicine}

This review focuses on "nanoparticles" among various nanostructures (carbon nanotubes, dendrimers, liposomes, micelles, nanographenes, nanorobots, and nanosheets) used in the field of nanomedicine. Properties and applications of other nanostructure that could not be presented in this review have been reviewed in detail in other reports. ${ }^{246-250}$

\section{Application of Surfactant-Coated Nanoparticles in Food Nanotechnology \\ Food Nanotechnology}

The development in the field of nanotechnology has been remarkable, and interest in its application in the global food industry has increased greatly in recent years due to its potential to add new properties and functions to existing food products. The international symposium "Nanotechnology Research: Applications in Nutritional Sciences" was held at Experimental Biology 2009, focusing on the application of nanotechnology to food and nutrition, and this field was widely recognized. ${ }^{251}$ Although a definition of nano-based technologies in the field of food and nutrition has not yet been established, the application of nanoscale material properties to the food and nutrition industry is generally named as "food nanotechnology". ${ }^{252-254}$ Currently, there are no established global rules regarding the applications of food nanotechnology, however, the Organization for Economic Cooperation and Development (OECD) launched a sponsorship program for testing of nanomaterials in 2007, and the FDA issued four guidance documents on the use of nanotechnology in animal products, cosmetics, food, and other products in 2014-2015. ${ }^{253}$ In the field of food nanotechnology, the approach of nanoparticles is considered to have the potential to be applied in various technologies, such as pesticides, ${ }^{255}$ antimicrobial, ${ }^{256}$ anti-solidification, ${ }^{257}$ plant genetic engineering, ${ }^{258}$ detection of foodborne pathogens, ${ }^{259}$ food processing, ${ }^{260}$ development of functional foods, ${ }^{261}$ purification of drinking water, ${ }^{262}$ extension of food preservation, ${ }^{263}$ and texture improvement ${ }^{252-254,264,265}$ (Figure 6). Surfactant-coated nanoparticles have been widely used in the field of food nanotechnology. Recent progress of surfactant-coated nanoparticles in this field is summarized in Table 2. Surfactant-coated organic and inorganic nanoparticles in food nanotechnology will be discussed separately in the following sections.

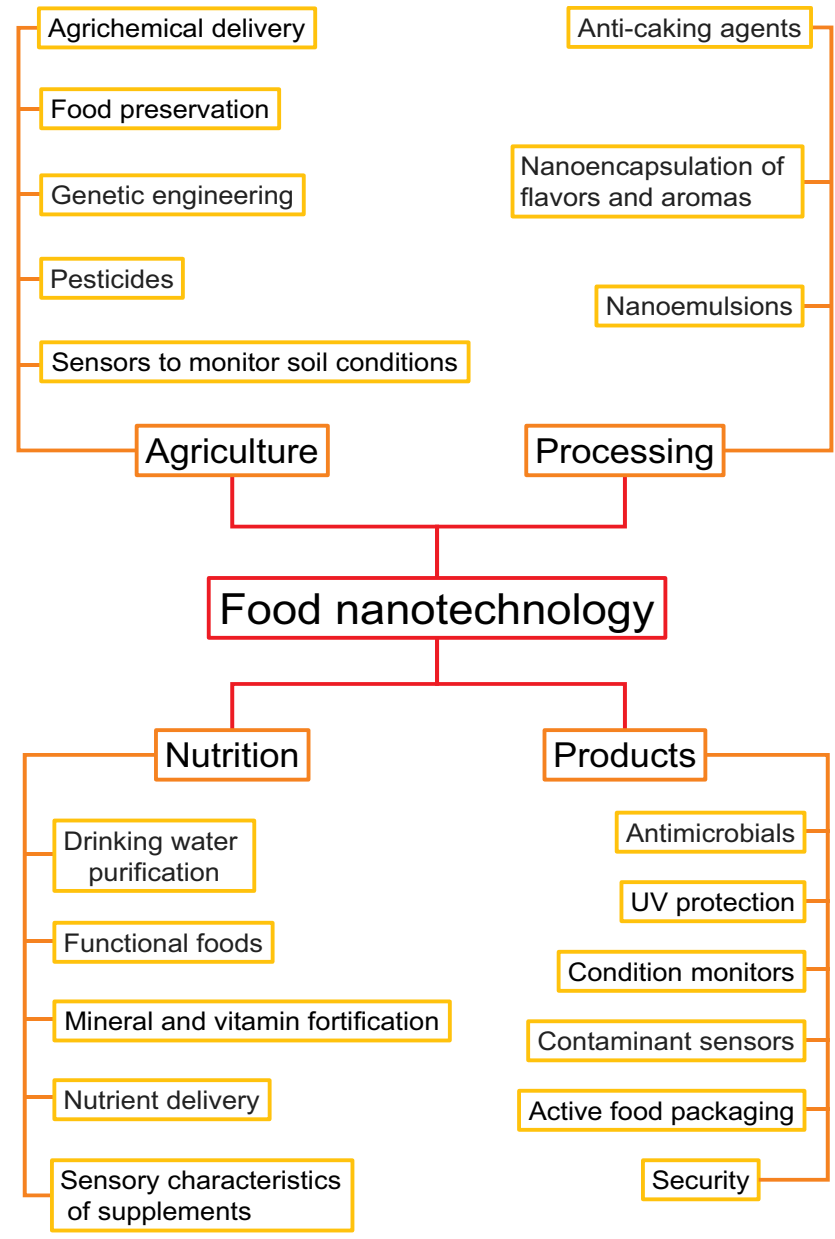

Figure 6 The potential applications of food nanotechnology.

Notes: Data from Martirosyan. ${ }^{265}$ 


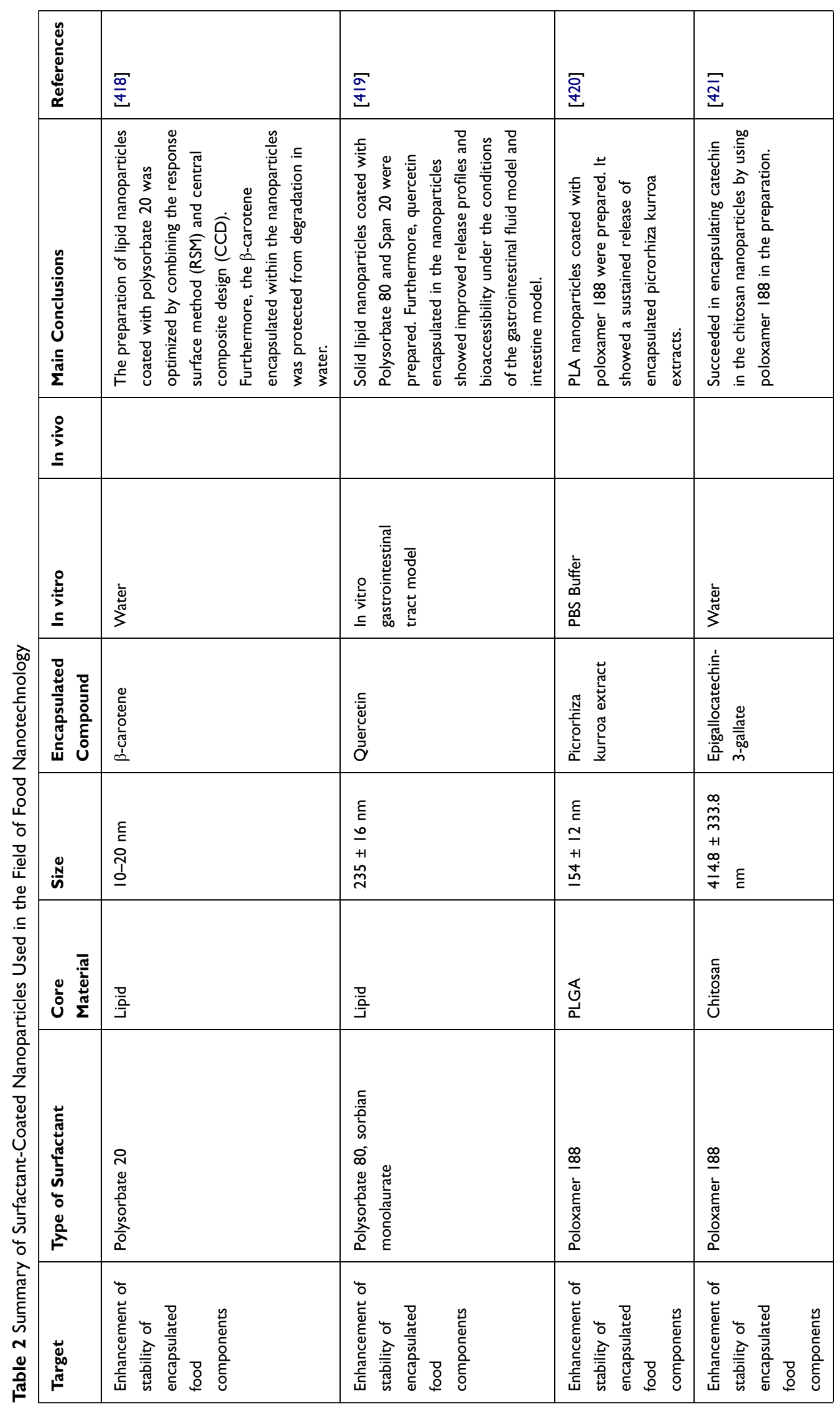




\begin{tabular}{|c|c|c|c|}
\hline $\mathbb{\Xi}$ & $\stackrel{\bar{\Im}}{\bar{g}}$ & 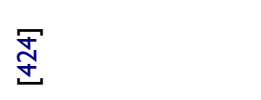 & $\stackrel{\Xi}{\nsubseteq}$ \\
\hline 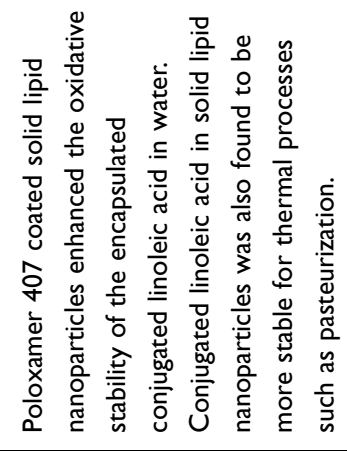 & 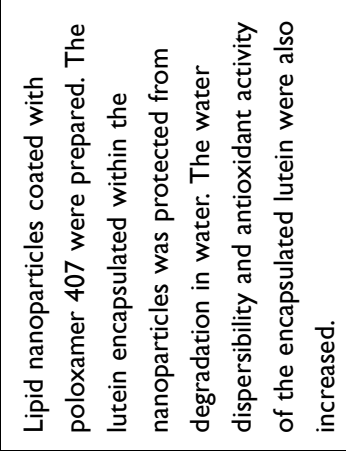 & 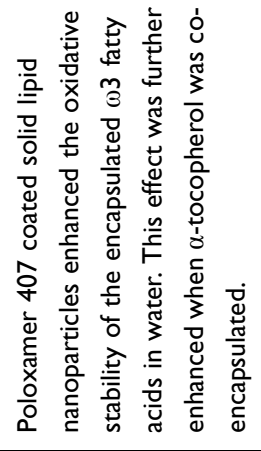 & 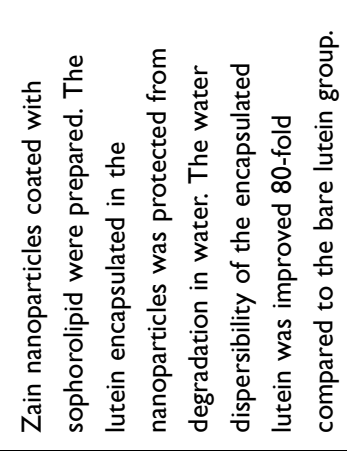 \\
\hline 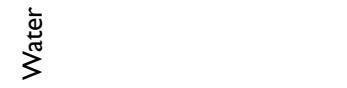 & 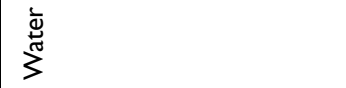 & 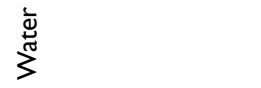 & 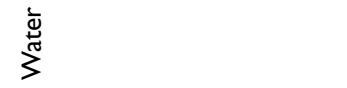 \\
\hline 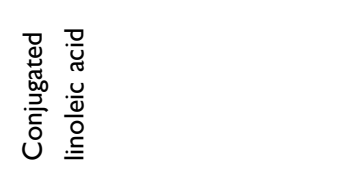 & 歖 & 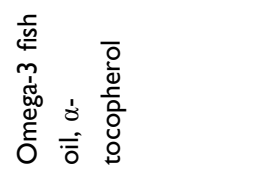 & 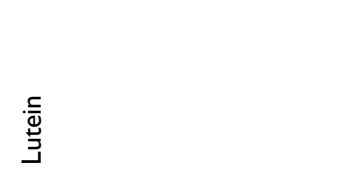 \\
\hline $\begin{array}{c}\underset{E}{E} \\
\Sigma\end{array}$ & 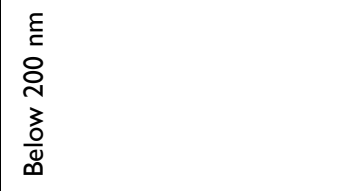 & 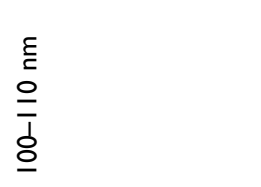 & 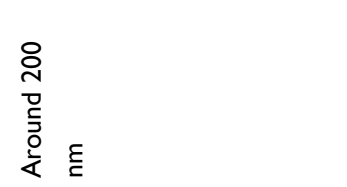 \\
\hline 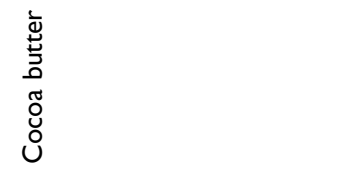 & $\frac{.00}{\bar{G}}$ & $\frac{0}{\frac{0}{G}}$ & 离 \\
\hline 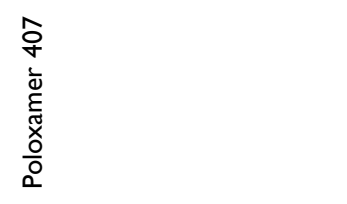 & 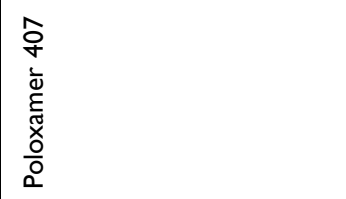 & 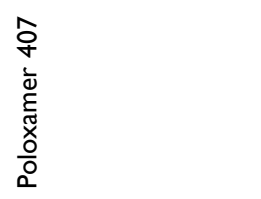 & $\begin{array}{l}\frac{0}{0} \\
\frac{\overline{0}}{\overline{0}} \\
\frac{0}{0} \\
\frac{0}{0} \\
0 \\
0\end{array}$ \\
\hline 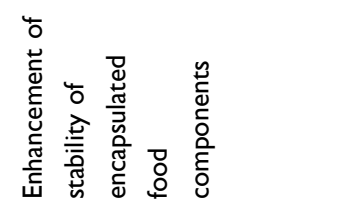 & 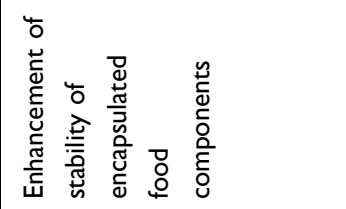 & 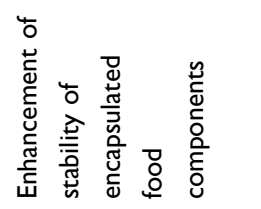 & 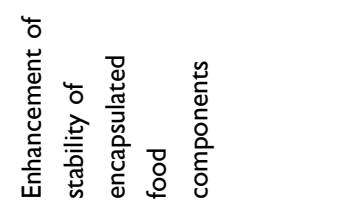 \\
\hline
\end{tabular}




\begin{tabular}{|c|c|c|c|c|c|}
\hline 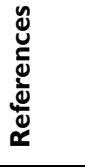 & $\begin{array}{l}\stackrel{\Xi}{ \pm} \\
\stackrel{5}{ }\end{array}$ & $\underset{\underset{I}{ \pm}}{\mathbb{I}}$ & 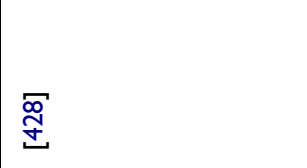 & $\frac{\sigma}{n}$ & 胥 \\
\hline 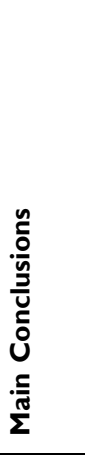 & 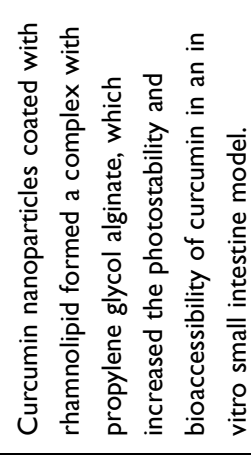 & 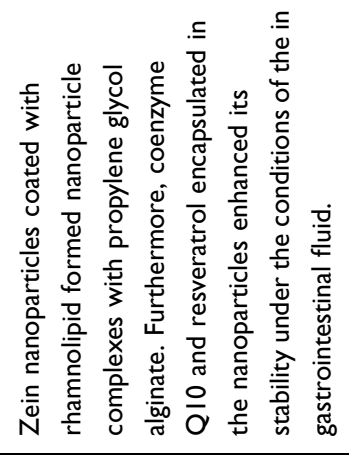 & 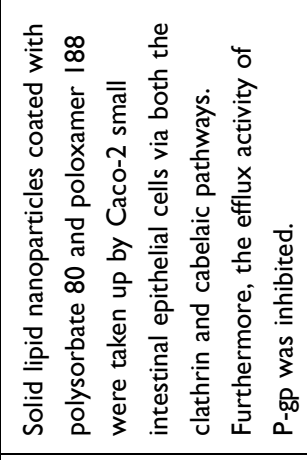 & 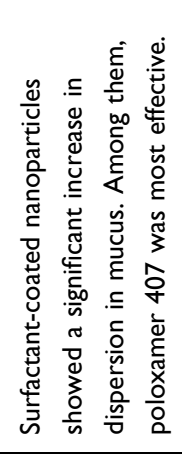 & 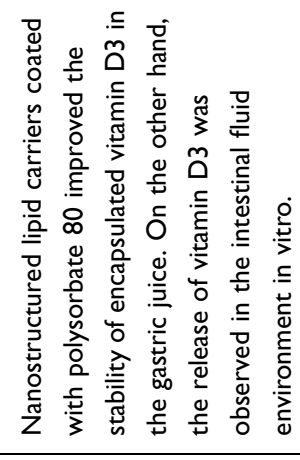 \\
\hline \multicolumn{6}{|l|}{$\begin{array}{l}\stackrel{?}{\lambda} \\
\underline{\underline{\Sigma}} \\
\end{array}$} \\
\hline 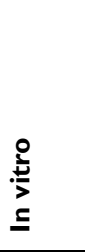 & 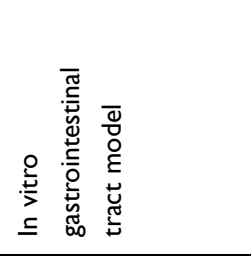 & 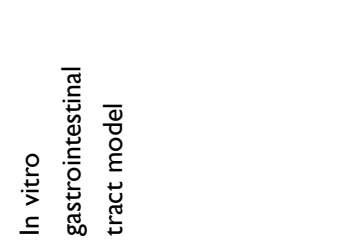 & 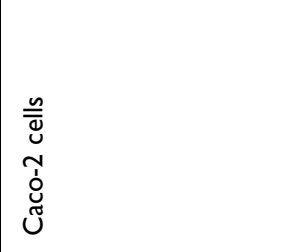 & 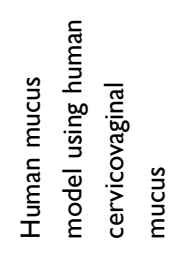 & 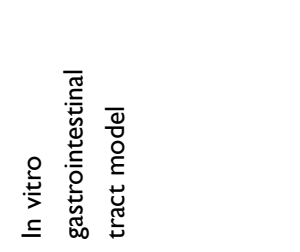 \\
\hline 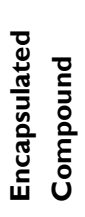 & 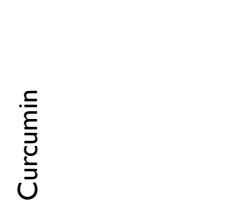 & 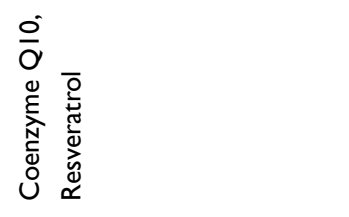 & & & 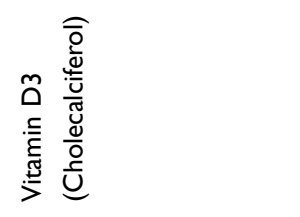 \\
\hline$\frac{N}{n}$ & 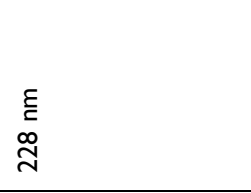 & 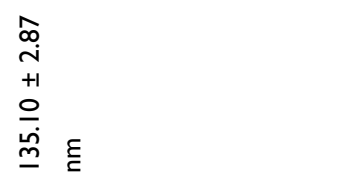 & 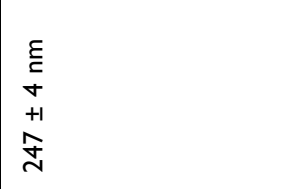 & 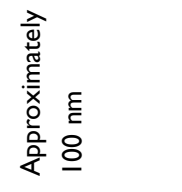 & 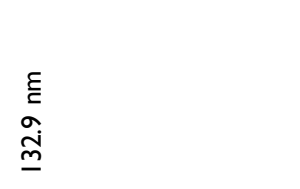 \\
\hline ن & 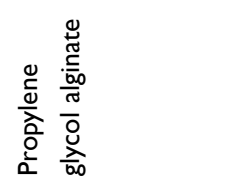 & 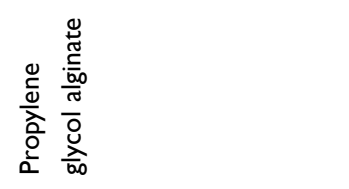 & : & 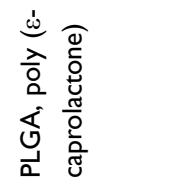 & $\frac{\overline{0}}{\bar{a}}$ \\
\hline 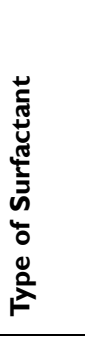 & 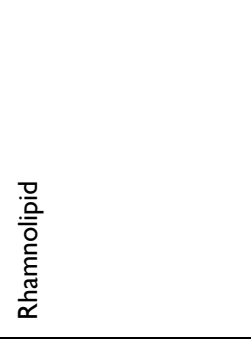 & 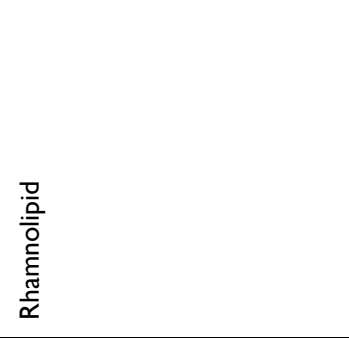 & 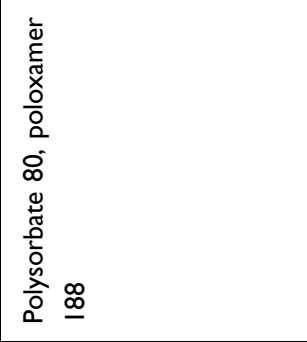 & 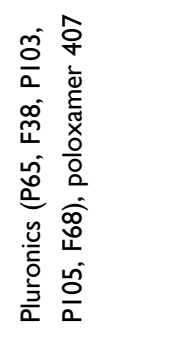 & 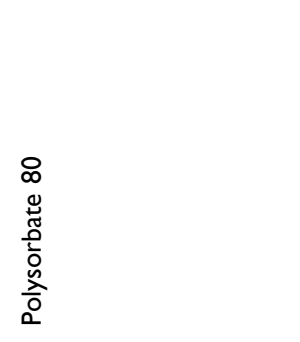 \\
\hline 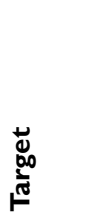 & 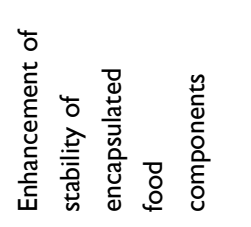 & 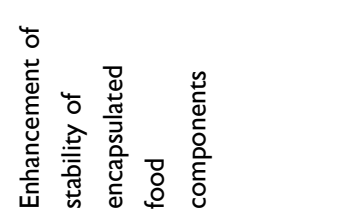 & 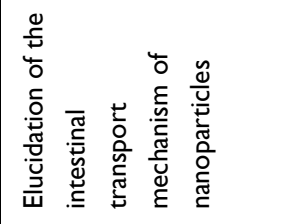 & 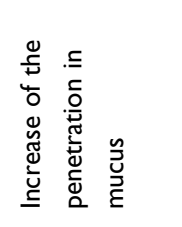 & 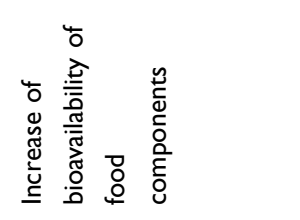 \\
\hline
\end{tabular}




\begin{tabular}{|c|c|c|c|c|}
\hline 官 & $\overline{\bar{g}}$ & $\underset{\widetilde{J}}{\stackrel{\widetilde{J}}{\Psi}}$ & 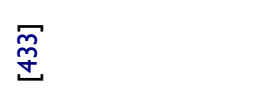 & 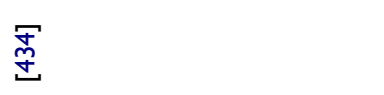 \\
\hline 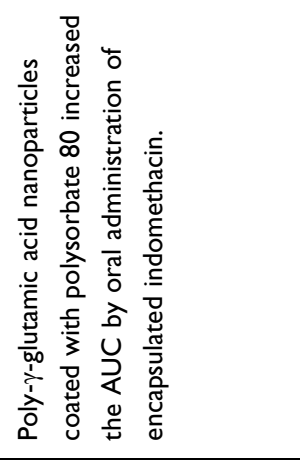 & 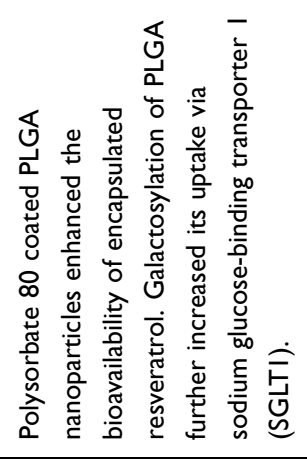 & 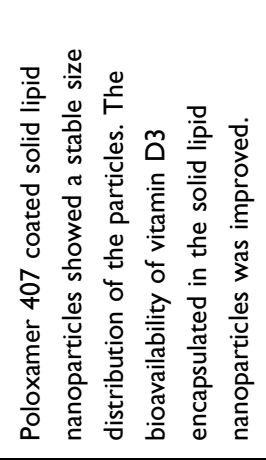 & 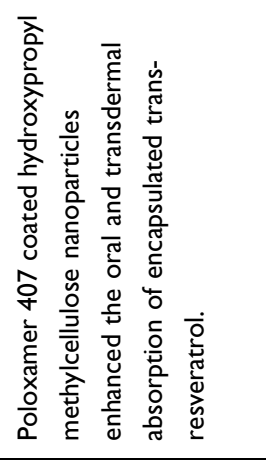 & 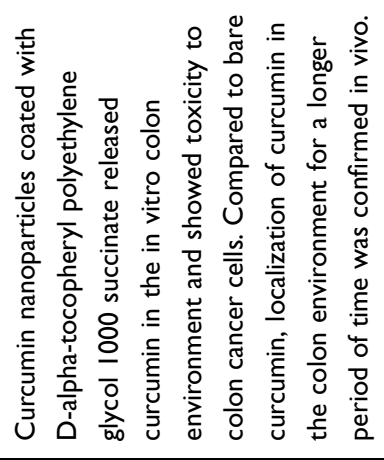 \\
\hline \multirow[t]{2}{*}{ 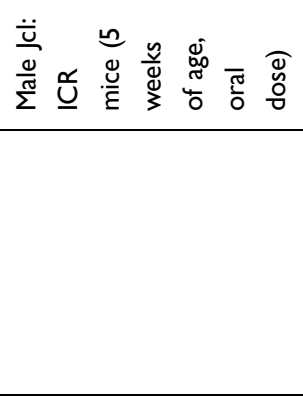 } & 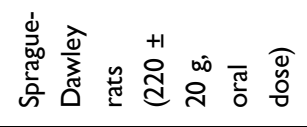 & 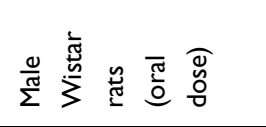 & 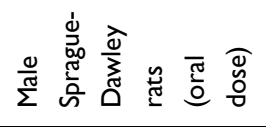 & 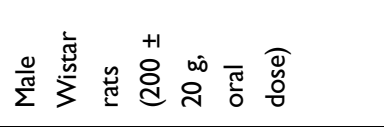 \\
\hline & & & & 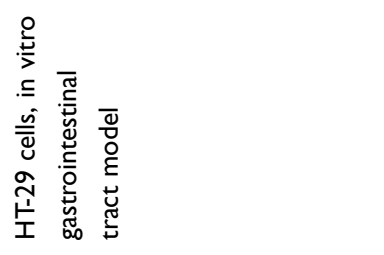 \\
\hline 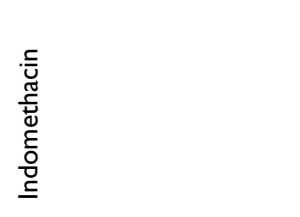 & 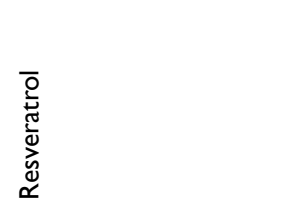 & 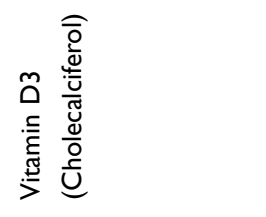 & 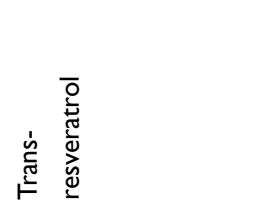 & \\
\hline 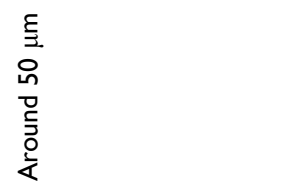 & 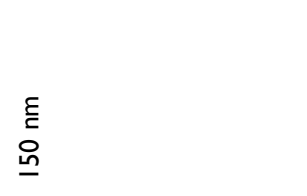 & $\begin{array}{l}\varepsilon \\
\varepsilon \\
\frac{\varepsilon}{0} \\
\frac{3}{0} \\
\frac{o}{0} \\
\infty\end{array}$ & $\begin{array}{l}\xi \\
\varepsilon \\
o \\
o \\
m \\
3 \\
\frac{0}{0} \\
\infty\end{array}$ & $\begin{array}{l}\varepsilon \\
\underline{E} \\
0 \\
+1 \\
\stackrel{M}{\underline{I}}\end{array}$ \\
\hline 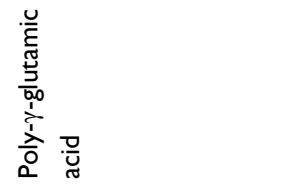 & 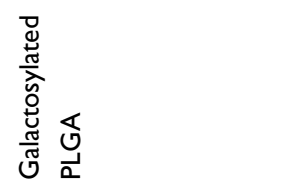 & : & 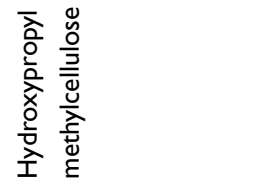 & 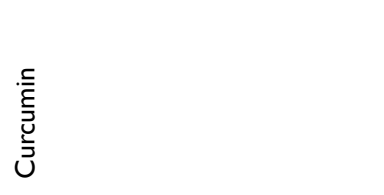 \\
\hline 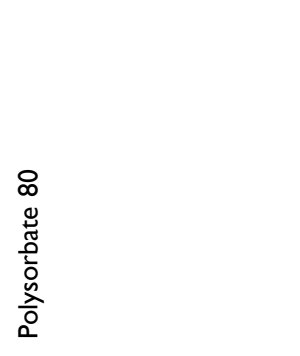 & 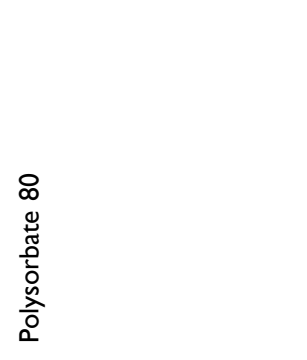 & 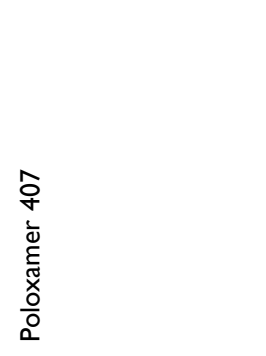 & 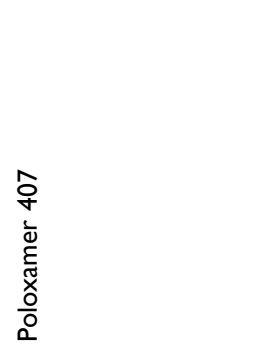 & 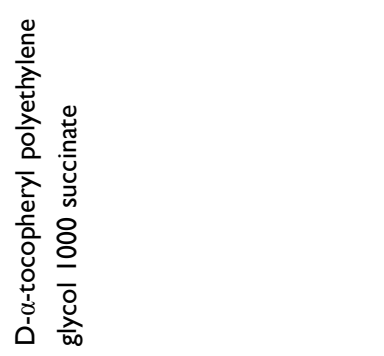 \\
\hline 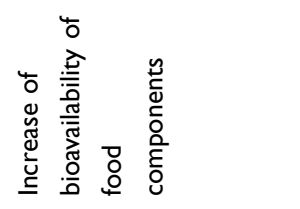 & 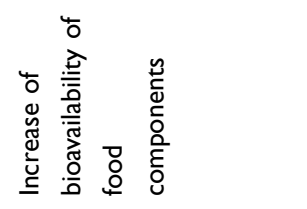 & 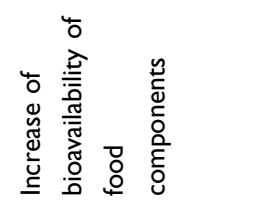 & 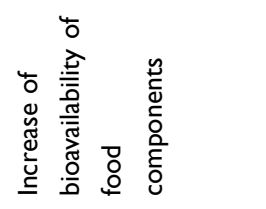 & 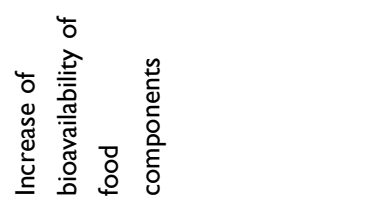 \\
\hline
\end{tabular}




\begin{tabular}{|c|c|c|c|c|}
\hline 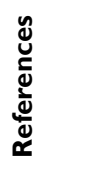 & $\stackrel{\mathscr{n}}{\mathscr{y}}$ & 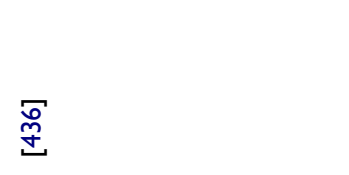 & $\underset{\text { 学 }}{ }$ & 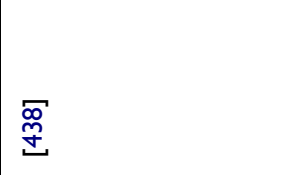 \\
\hline 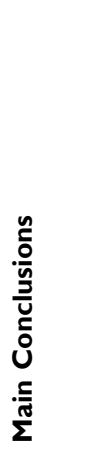 & 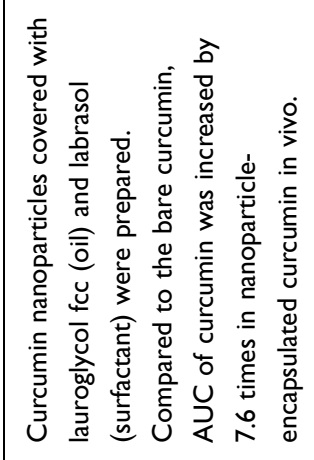 & 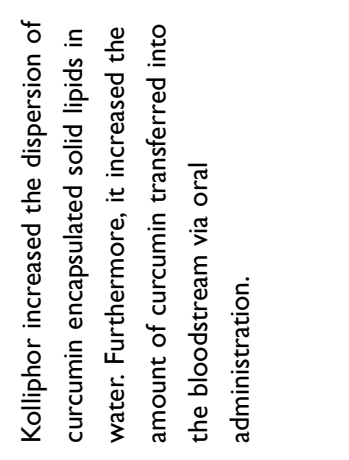 & 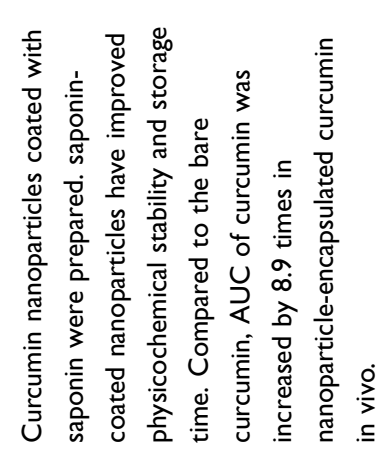 & 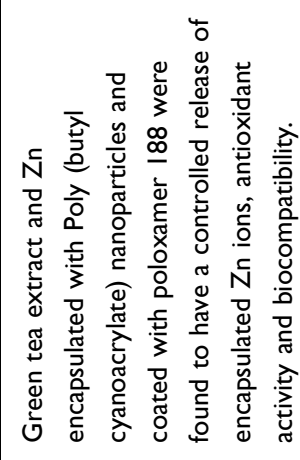 \\
\hline $\begin{array}{l}\stackrel{0}{>} \\
\underline{\underline{x}}\end{array}$ & 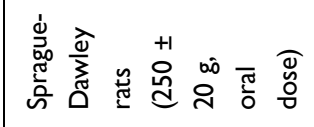 & 离 & 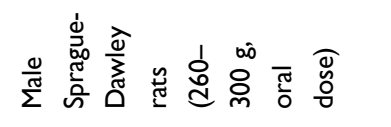 & \\
\hline 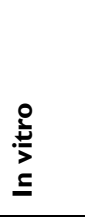 & & & 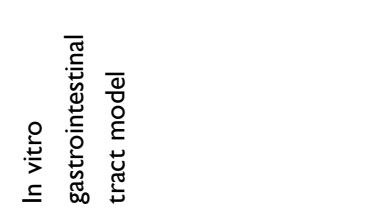 & $\begin{array}{l}\frac{n}{\underline{\underline{U}}} \\
\sum_{\tilde{w}}^{\tilde{N}}\end{array}$ \\
\hline 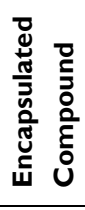 & & 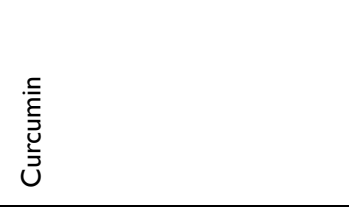 & & 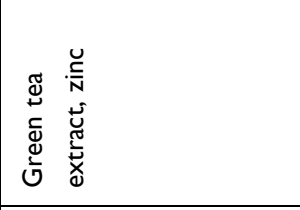 \\
\hline$\stackrel{N}{n}$ & 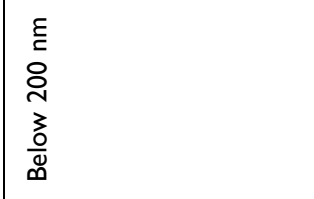 & 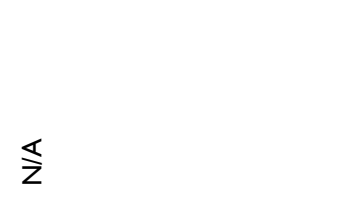 & $\begin{array}{l}\varepsilon \\
E \\
o \\
\frac{0}{1} \\
\\
\end{array}$ & 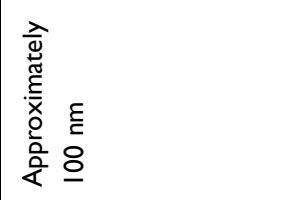 \\
\hline ن & 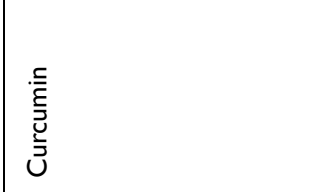 & : & 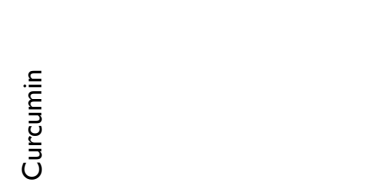 & 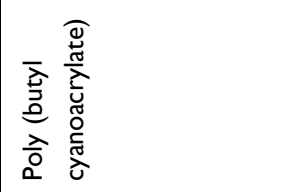 \\
\hline 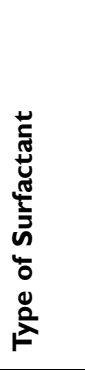 & 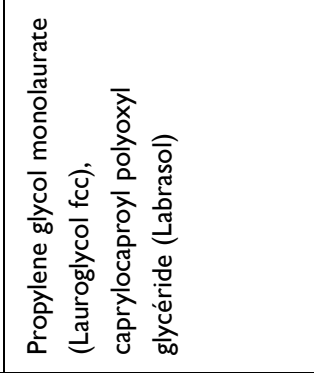 & $\begin{array}{l}\underline{n} \\
\underline{\underline{1}} \\
\bar{o} \\
\frac{\bar{c}}{0} \\
\underline{\overline{\overline{0}}} \\
\underline{\underline{a}}\end{array}$ & 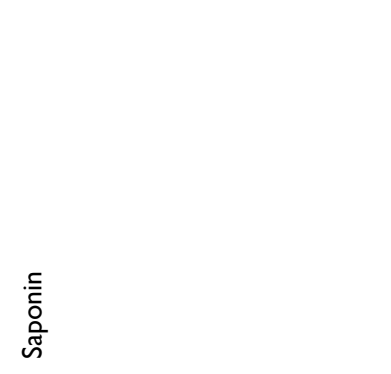 & 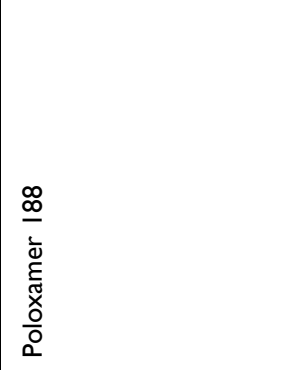 \\
\hline 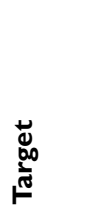 & 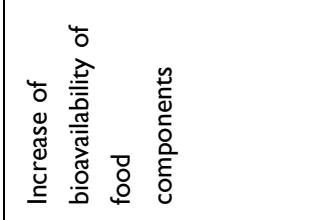 & 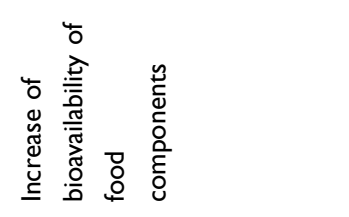 & 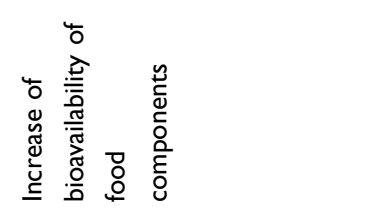 & 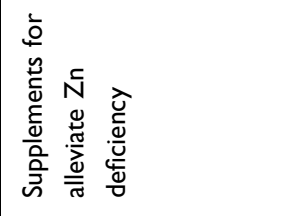 \\
\hline
\end{tabular}




\begin{tabular}{|c|c|c|c|c|}
\hline 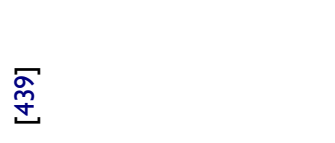 & 离 & 筧 & $\overline{\bar{J}}$ & $\underset{\mathbb{J}}{\mathbb{g}}$ \\
\hline 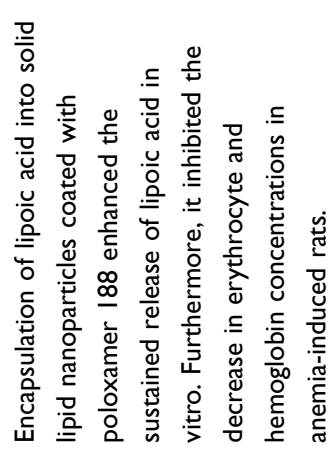 & 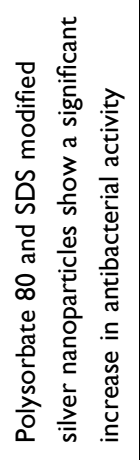 & 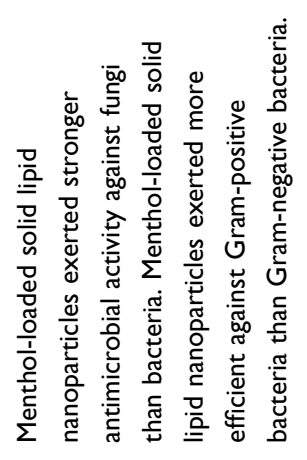 & 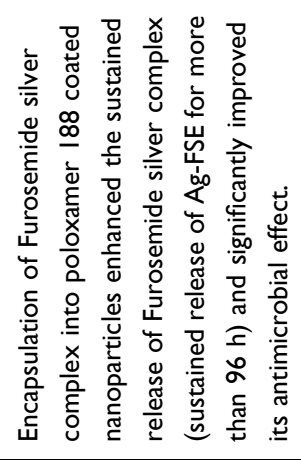 & 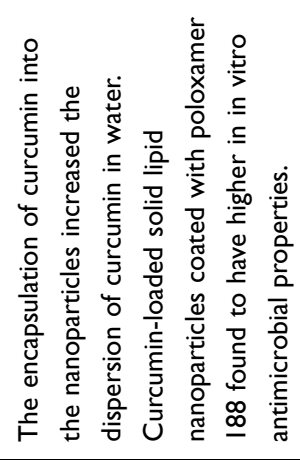 \\
\hline \multicolumn{5}{|l|}{ 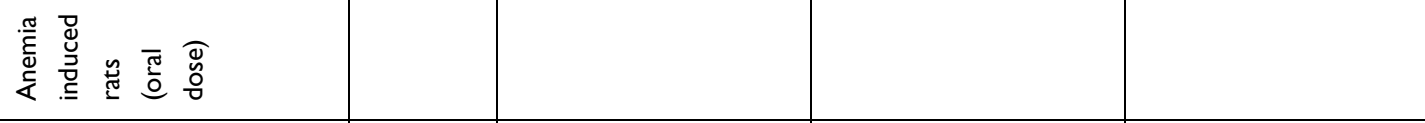 } \\
\hline & 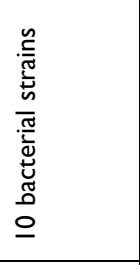 & 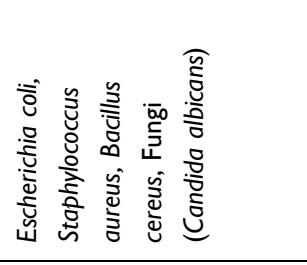 & 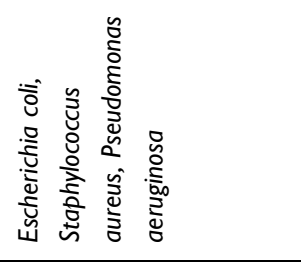 & 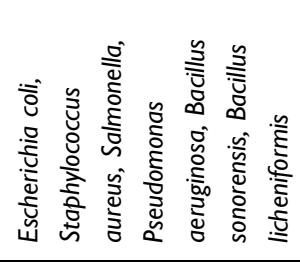 \\
\hline 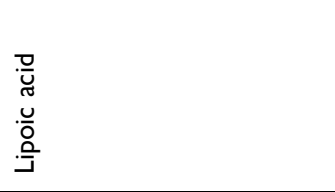 & & $\begin{array}{l}\overline{0} \\
\frac{\bar{c}}{\bar{v}} \\
\frac{\bar{v}}{\Sigma}\end{array}$ & 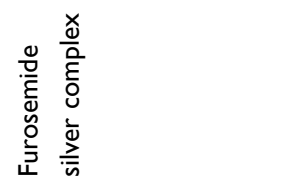 & 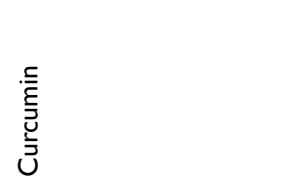 \\
\hline 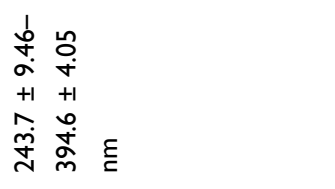 & 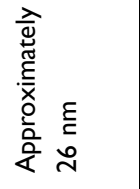 & 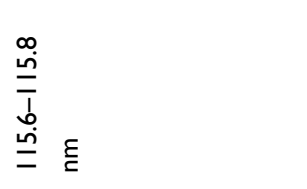 & 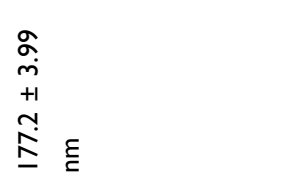 & $\begin{array}{c}\varepsilon \\
\underline{c} \\
\stackrel{\infty}{\underline{m}}\end{array}$ \\
\hline$\frac{.0}{\bar{a}}$ & $\frac{\grave{\Phi}}{\overline{\bar{n}}}$ & $\frac{.0}{\bar{a}}$ & $\frac{.0}{\bar{a}}$ & 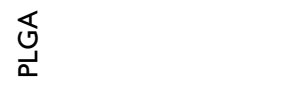 \\
\hline 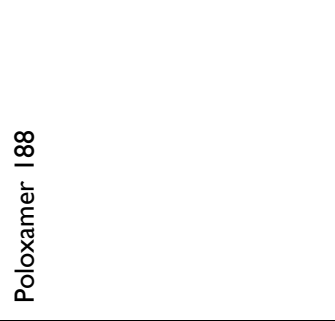 & 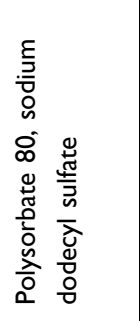 & 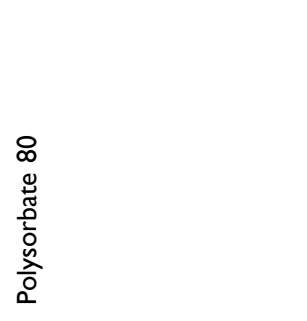 & 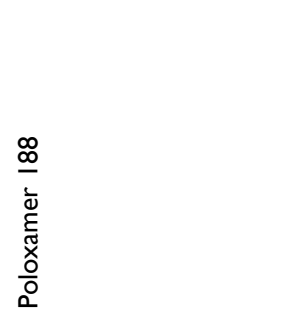 & 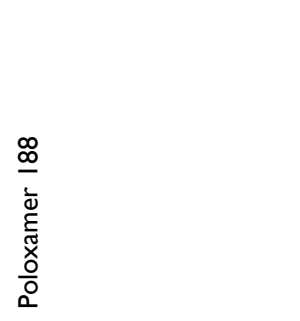 \\
\hline 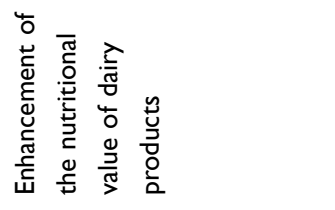 & 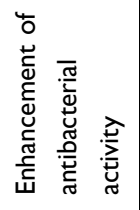 & 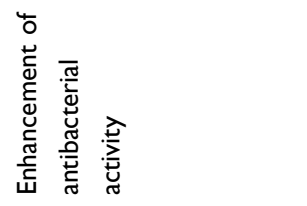 & 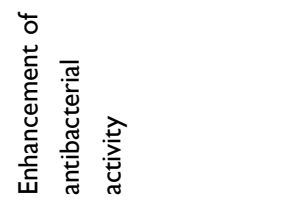 & 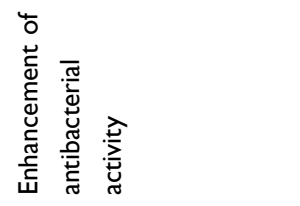 \\
\hline
\end{tabular}




\begin{tabular}{|c|c|c|c|c|c|}
\hline 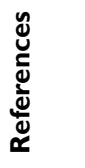 & 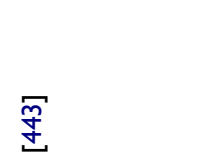 & $\underset{+}{\mathbb{F}}$ & $\stackrel{\square}{\frac{f}{4}}$ & $\stackrel{?}{\stackrel{P}{+}}$ & $\underset{+}{\mathbb{f}}$ \\
\hline 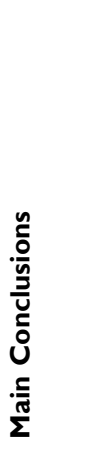 & 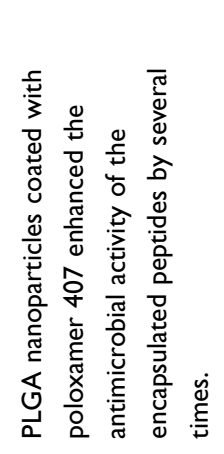 & 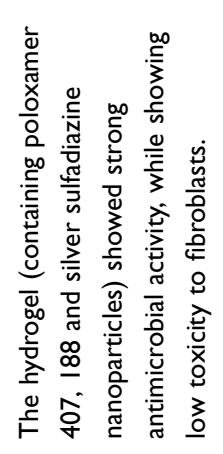 & 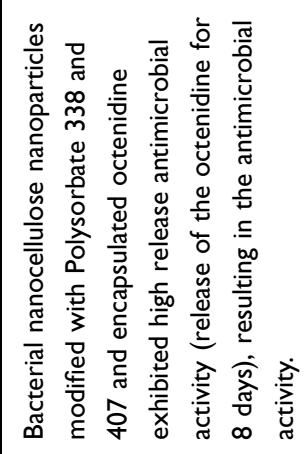 & 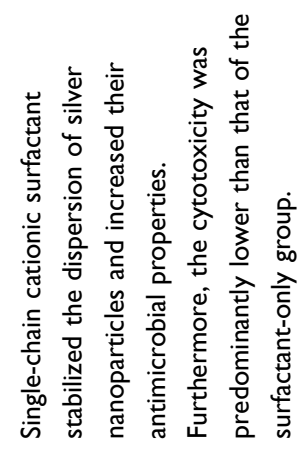 & 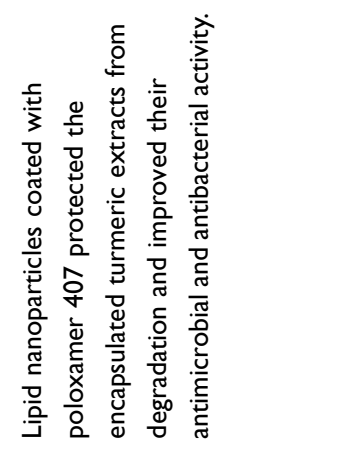 \\
\hline 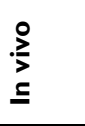 & & & & & \\
\hline 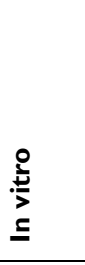 & 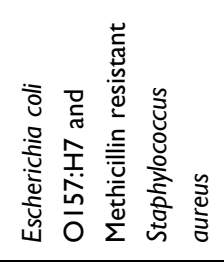 & 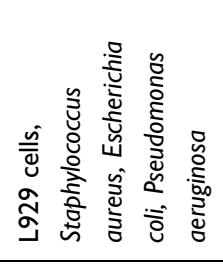 & 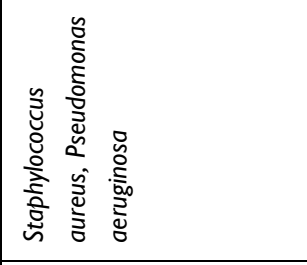 & 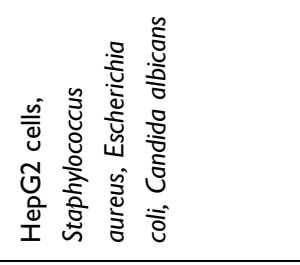 & 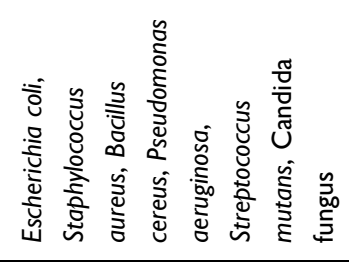 \\
\hline 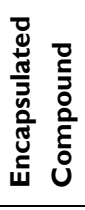 & 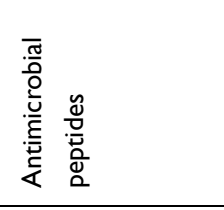 & & 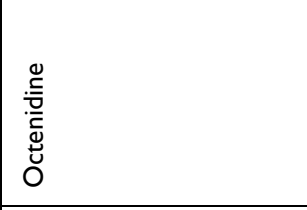 & & 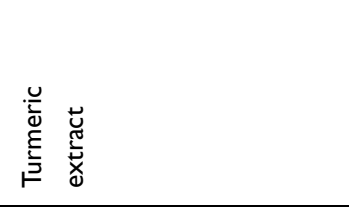 \\
\hline$\frac{N}{n}$ & 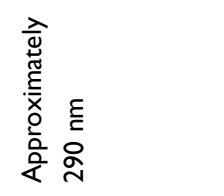 & $\begin{array}{l}\varepsilon \\
\underline{c} \\
o \\
\text { ò }\end{array}$ & $\begin{array}{l}\varepsilon \\
\varepsilon \\
0 \\
3 \\
\frac{0}{0} \\
\infty\end{array}$ & $\begin{array}{l}\varepsilon \\
\varepsilon \\
o \\
\frac{\varepsilon}{3} \\
\frac{0}{\Phi} \\
\infty\end{array}$ & $\frac{\varepsilon}{\underline{I}}$ \\
\hline نे & 志 & 竞 & 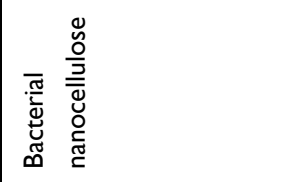 & $\frac{\stackrel{\bar{\nu}}{\bar{\nu}}}{\overline{\bar{\omega}}}$ & $\frac{\overline{0}}{\bar{a}}$ \\
\hline 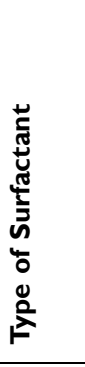 & 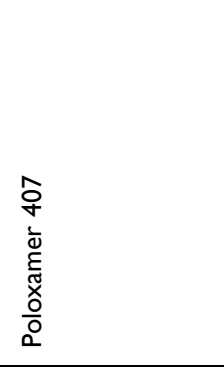 & 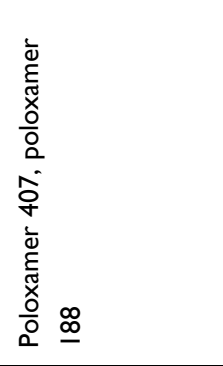 & 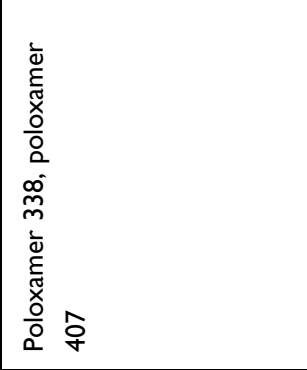 & 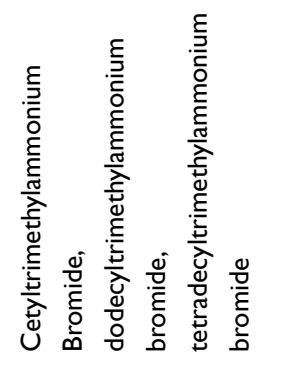 & 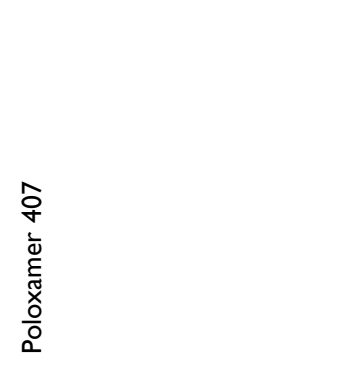 \\
\hline 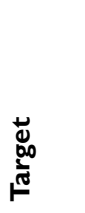 & 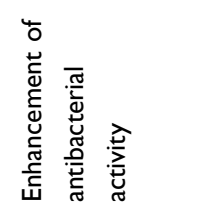 & 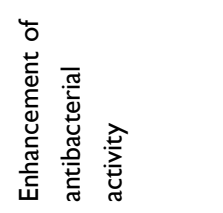 & 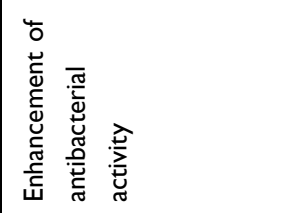 & 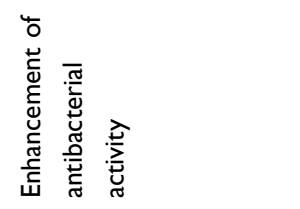 & 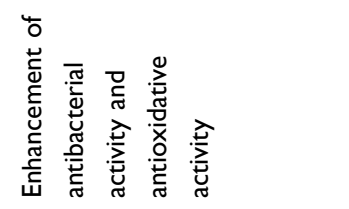 \\
\hline
\end{tabular}




\begin{tabular}{|c|c|c|c|c|}
\hline 离 & 㒵 & $\begin{array}{l}\text { 总 } \\
\text { 要 }\end{array}$ & $\overline{\bar{g}}$ & 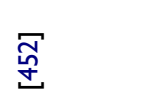 \\
\hline 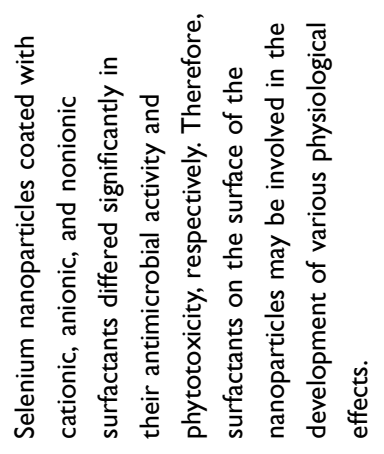 & 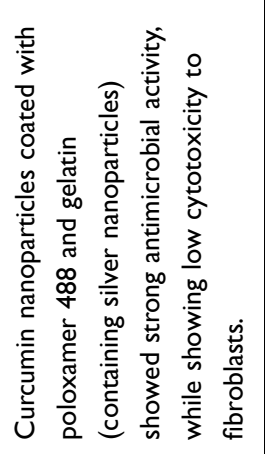 & 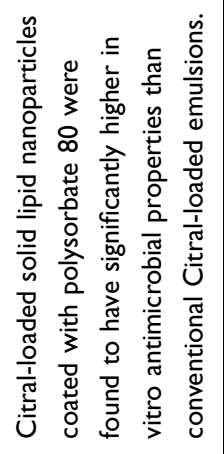 & 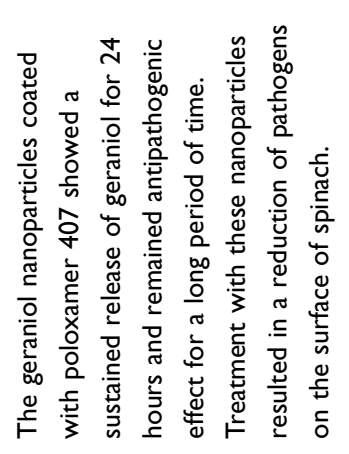 & 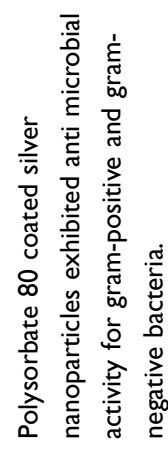 \\
\hline 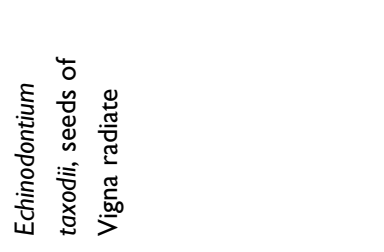 & 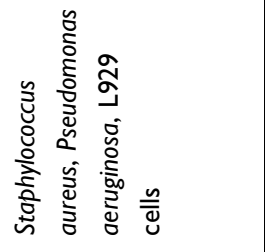 & 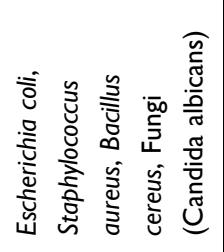 & 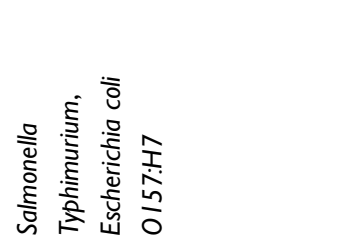 & 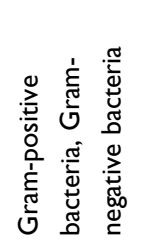 \\
\hline & & $\begin{array}{l}\overline{\tilde{U}} \\
\overline{\tilde{U}}\end{array}$ & & \\
\hline $\begin{array}{l}\varepsilon \\
\text { E } \\
\text { సิ } \\
\text { ஸे }\end{array}$ & 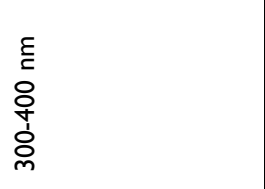 & 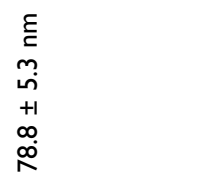 & 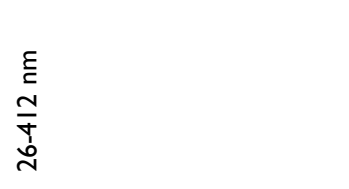 & $\begin{array}{l}\varepsilon \\
\text { E } \\
\text { i }\end{array}$ \\
\hline 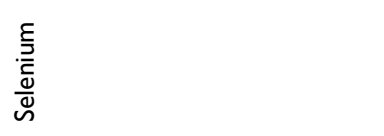 & $\begin{array}{l}\frac{\varepsilon}{\bar{E}} \\
\frac{\vec{\omega}}{\mathscr{N}} \\
心\end{array}$ & : & 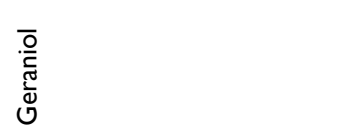 & $\frac{\grave{\bar{\Phi}}}{\grave{i n}}$ \\
\hline 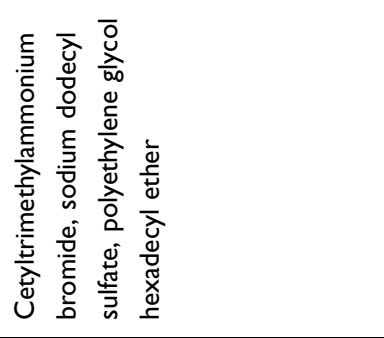 & 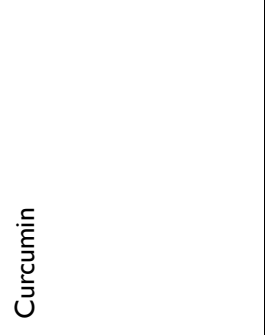 & 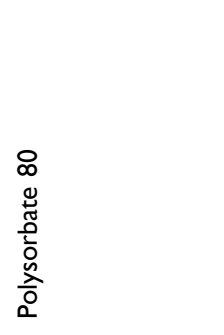 & 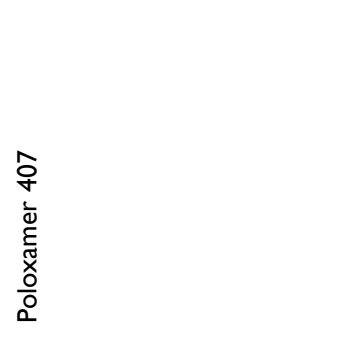 & 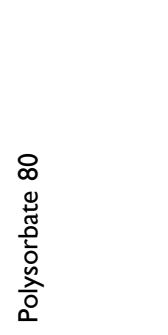 \\
\hline 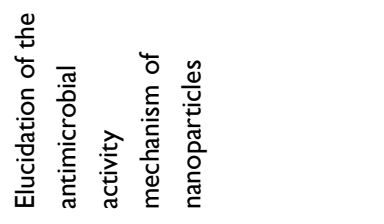 & 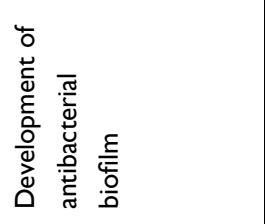 & 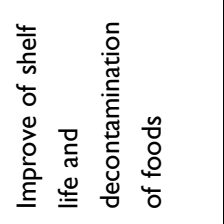 & 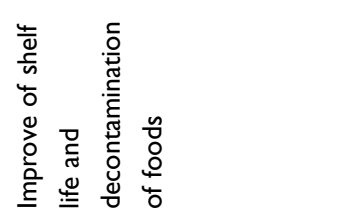 & 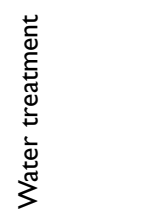 \\
\hline
\end{tabular}




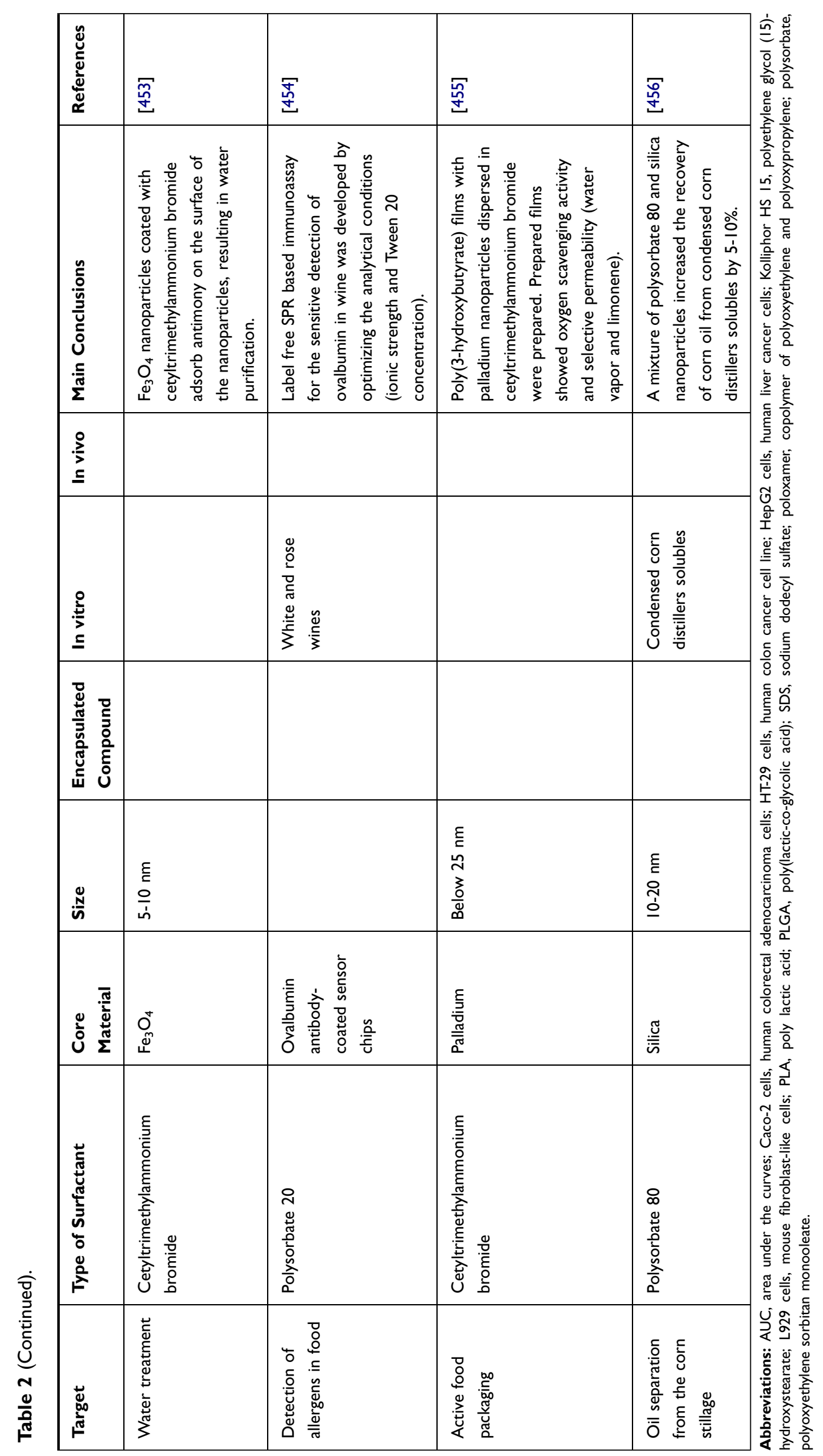




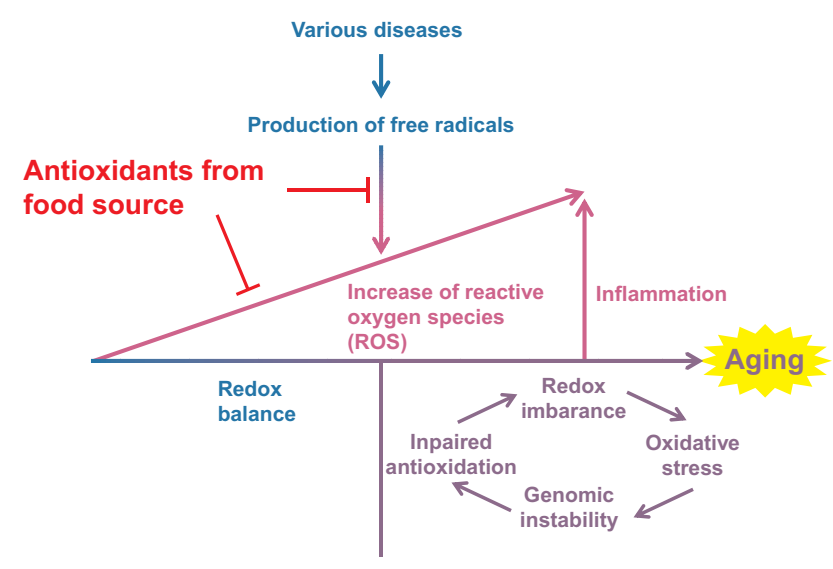

Figure 7 Illustration of the relationships between diseases, free radicals, reactive oxygen species, and aging in the body, and its regulation by antioxidants from food source.

Notes: Data from Miyazawa ${ }^{241}$ and Adapted from Elsevier Books, 191, Fajardo AM, Bisoffi M, Chapter 18 - Curcumin analogs, oxidative stress, and prostate cancer, 191-202, Copyright 2014, with permission from Elsevier. ${ }^{267}$.

\section{Surfactant-Coated Organic Nanoparticles in Food Nanotechnology Smart Food}

Food components are supplied to the human body on a daily basis via oral intake, and are maintained in the body at optimal concentrations in various tissues for life support. However, their concentrations fluctuate due to various disorders such as disease and aging, leading to the disruption of the redox balance in the body. Certain types of diseases and obesity also increase the amount of ROS produced in the human body, resulting in accelerated aging and disease progression (Figure 7). ${ }^{176,266,267}$ It is recognized that the concentration of antioxidants in human blood decreases with aging. Mecocci et al measured the concentrations of various antioxidants $(\alpha$-carotene, $\beta$-carotene, $\beta$-cryptoxanthin, lutein, zeaxanthin, all-trans lycopene, lycopene total, retinol, ascorbic acid, uric acid, $\alpha$ tocopherol, thiols, plasma superoxide dismutase [SOD], red blood cells [RBCs] SOD, glutathione peroxidase [GPX] and nicotinamide adenine dinucleotide phosphate [NADPH]) in the blood of healthy subjects under 60 , $61-80,81-99$, and over 100 years of age, and reported that several food-derived antioxidants $(\alpha$-carotene, $\beta$-carotene, $\beta$-cryptoxanthin, lutein, zeaxanthin, all-trans lycopene, lycopene total, ascorbic acid and uric acid) tended to decrease with age (Figure 8). ${ }^{268}$ It is hoped that these problems can be overcome by daily dietary intake of foodderived antioxidants to achieve longevity (Figure 7). Research on the inhibition of disease progression and development by daily intake of food components has been widely conducted. For example, it has been reported that the blood of dementia patients with Alzheimer's disease has higher concentrations of RBCs with high levels of phospholipid hydroperoxides in their lipid membranes (also named as "aged RBCs" which are responsible for poor oxygenation and deterioration of blood rheology) compared to healthy subjects. ${ }^{269-272}$ Since the presence of these aged RBCs are considered to be one of the causes of the progression and onset of Alzheimer's disease, food ingredients that prevent the peroxidation of red blood cell membrane lipids are expected. Nakagawa et al reported that daily intake of polar carotenoid (astaxanthin [6 or 12 $\mathrm{mg} /$ day] or lutein [9.67 $\mathrm{mg} /$ day]) capsules for more than two weeks suppressed the appearance of aged RBCs in human blood. ${ }^{273,274}$ Obesity is regarded as a low-grade inflammatory disease. ${ }^{275}$ Miyazawa et al reported that 20 weeks of simultaneous intake of polyphenol (curcumin [1 $\mathrm{g} / \mathrm{kg}$ diet in this study], which is abundant in turmeric) and alkaloid (piperine [50 mg/kg diet in this study], enhancer of curcumin's bioavailability, which is abundant in pepper) reduced inflammation (interleukin [IL]-1 $\beta$ and keratinocyte chemoattractant/growth-regulated oncogene chemokines [KC/GRO] in plasma) and body fat in obese under caloric restriction model (C57BL/6) mice. ${ }^{276}$ Gregor et al reported that a 3-week intake of a vitamin $\mathrm{E}$ analog (rice bran tocotrienol [5 or $10 \mathrm{mg} / \mathrm{day}]$ ) reduced triglyceride and phospholipid hydroperoxide levels in the blood and liver in F344 rats. ${ }^{277}$ Vitamin C has been around for 100 years since its discovery. Its prevailing theory of its mechanism of anticancer effect was due to cytotoxicity caused by hydrogen peroxide $\left(\mathrm{H}_{2} \mathrm{O}_{2}\right)$ produced by the oxidation of vitamin $\mathrm{C}$ (ascorbic acid) in extracellular environment. $^{278}$ On the other hand, Yun et al reported in 2014 a new theory in which the oxidized product of ascorbic acid (dehydroascorbic acid) is taken up by cancer cells via glucose transporter (GLUT) and inhibit the production of adenosine triphosphate (ATP) and lead the cell death to cancer. ${ }^{279}$ Since this report, there has been a surge of interest in elucidating more detailed mechanisms of the anticancer effects of vitamin C. ${ }^{280,281}$ As introduced in section "Food Nanotechnology" in this review, nanotechnology has been expected to have a variety of applications in the fields of basic research and industry of foods. Not only that, attempts to maximize the useful effects of various food ingredients such as those described above using nanotechnology have also attracted attention. Nanotechnology is being developed to encapsulate food 

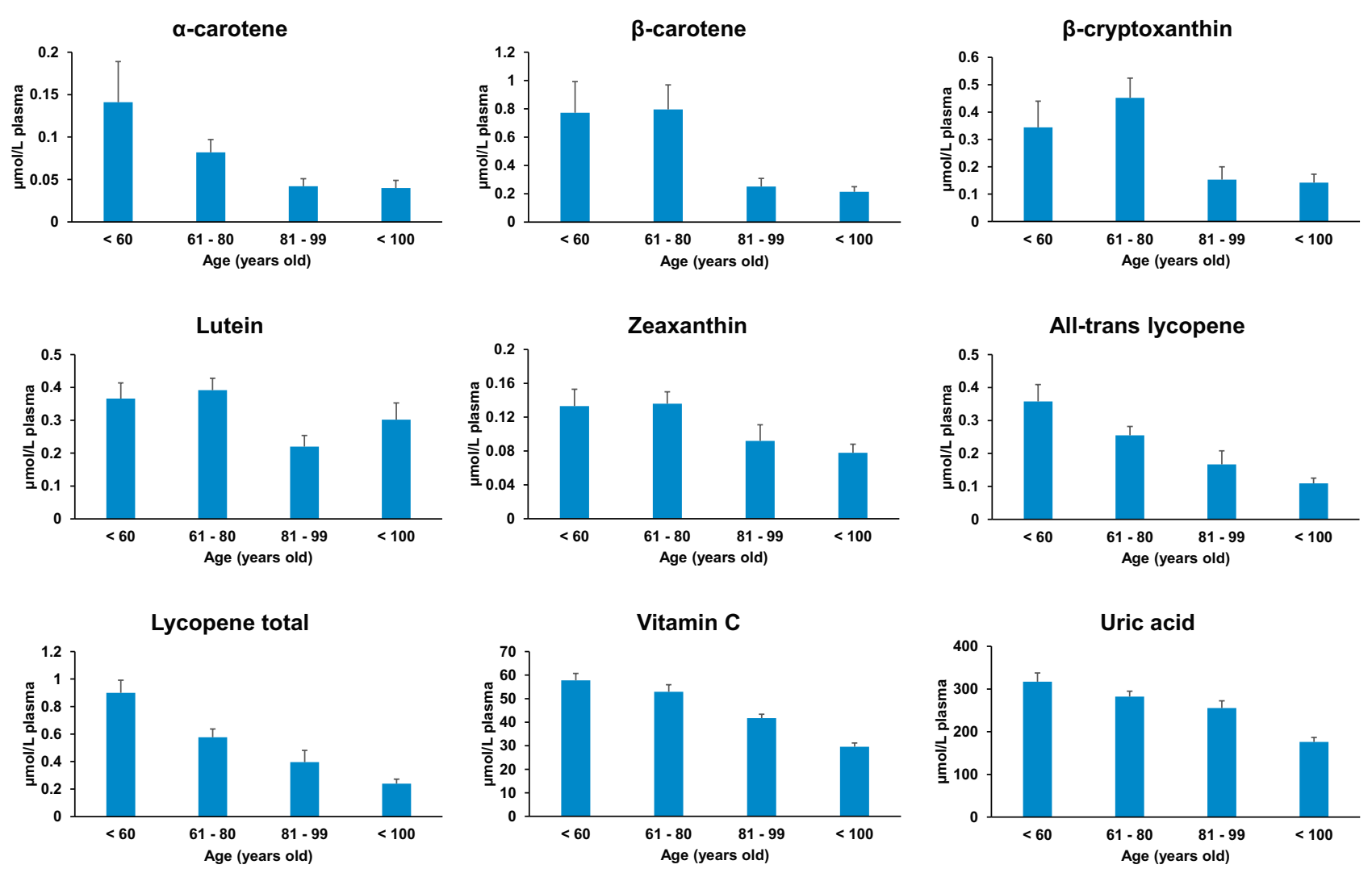

Figure 8 Age-related decrease in plasma concentrations of antioxidants from food source. Notes:Data from Mecocci.$^{268}$

components into organic nanoparticles to perform various functions. This attempt to improve human health was named "Smart food" by Martínez-Ballesta et al. ${ }^{282,283}$ The components of the nanoparticles used in smart food are selected to have low toxicity in living organisms. Typical examples include polymers (chitosan, collagen, gelatin, hyaluronic acid, and PLGA), solid lipids (cholesterol, palmitic acid and stearic acid), and proteins (milk protein, nisin, and zein) (Figure 2B). ${ }^{284-286}$ It will be of great significance if this smart food can extend healthy life expectancy through daily dietary habits. Davis et al reported a decrease of nearly half in the content of useful antioxidants in crops in 1999 compared to $1950 .{ }^{287}$ From the perspective of the food crisis, there will be great value in this smart food that can efficiently supply nutrients to the body.

\section{Application of Food Nanotechnology in the Digestive System}

Encapsulating food ingredients into nanoparticles extends their shelf life and protects them from degradation in the digestive system. ${ }^{288-290}$ Dietary patterns, food matrix, and passage through the gastrointestinal tract were often important factor for food nanotechnology. These factors may have a major impact on nanoparticle characteristics, behavior, and toxicity. ${ }^{291}$ As described briefly in section "Nonionic Surfactants in the Food Industry" in this review, the presence of surfactants occupies an important position in this mechanism of digestion and absorption. In complex of in vivo digestive system, not yet have an integrated knowledge of how the different types of surfactants used in surfactant-coated nanoparticles affect the above factors. However, these are important for understanding of the absorption and metabolism of nanoparticles, and their necessity has also been suggested in other reviews. ${ }^{292-294}$ The orally administered nanoparticles are known to avoid various digestive enzymes (such as amylase, lipase, and pepsin) and the highly acidic environment of the stomach (pH 1-3), reach the small intestinal epithelium, and are absorbed into the body (Figure 9). ${ }^{49,295,296}$ As a result, the bioavailability of the encapsulated food components in the nanoparticles is improved. ${ }^{260,261}$ The balance between the hydrophilic and hydrophobic nature of the nanoparticle interface and the digestive system is an important factor 


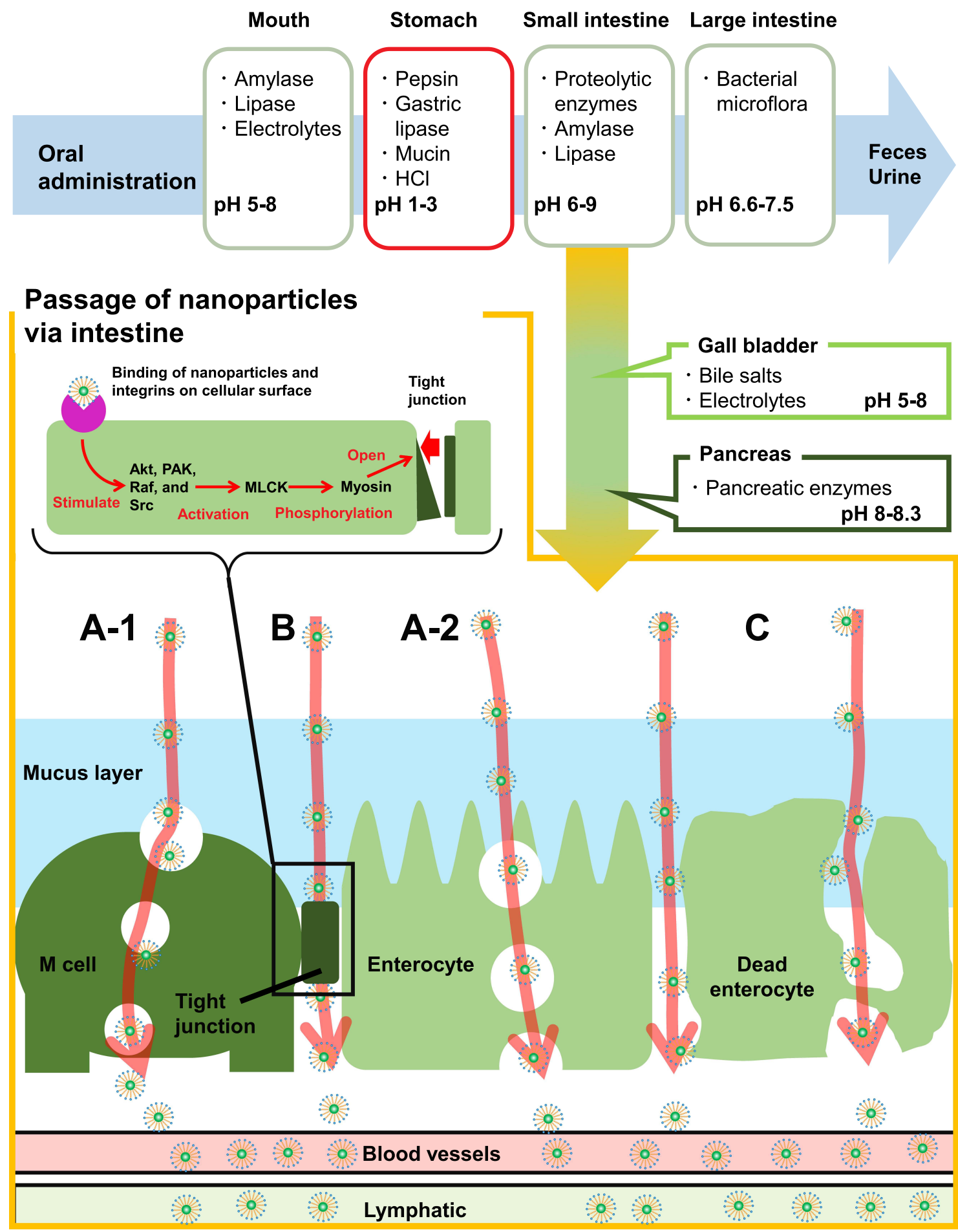

Figure 9 The digestive stages after oral administration and the mechanisms of in vivo uptake of surfactant-coated nanoparticles through the small intestine. Notes: (A-I) Transcellular route, through the M cells. (A-2) Transcellular route, through the enterocyte. (B) Paracellular route. (C) Persorption route. Data from these published studies 31829549

responsible for absorption into the body, and hydrophilic coating on the surface of hydrophobic nanoparticles has been widely accepted to increase their absorption. ${ }^{297-299}$
Maisel et al prepared polystyrene nanoparticles coated with a hydrophilic polymer (PEG) and confirmed their localization to the small intestine in ex vivo studies in 
mice. $^{300}$ The results showed that bare nanoparticles (unmodified nanoparticles) accumulated in the mucosal layer of the small intestine, whereas the PEG-coated nanoparticles reached closer to the small intestinal villi with a homogeneous distribution. Bourganis et al demonstrated a similar phenomenon in a transwell model using porcine mucosa. ${ }^{301}$ It has also been reported that coating the surface of nanoparticles with hydrophilic natural polysaccharides (chitosan) also increase the absorption of food components. This nanoparticle coating enhances mucosal adhesion and protects the nanoparticles from acidic environment and digestive enzymes. ${ }^{302,303}$ The digestive system is constantly secreting mucus, and a $15.5 \pm 4.5 \mu \mathrm{m}$ thick mucus barrier ( $\mathrm{pH}$ 5.5-7.5) physically blocks microorganisms and hydrophobic substances entering the intestinal tissues. ${ }^{304-306}$ This mucus forms a hydrogel (primarily composed of water and lipids, mucin, nucleic acids, and proteins) with a mucin skeleton cross-linked by hydrophobic interactions and disulfide bonds. ${ }^{307}$ Therefore, the nanoparticles that reach the small intestine must have the ability to penetrate the mucus barrier to reach the intestinal tissues and be absorbed into the body. It has been discovered that the surfactant-coated nanoparticles can pass through the mucus layer to reach the intestinal tissues (Figure 6A). Ensign et al reported that pretreatment with a nonionic surfactant (poloxamer 407) uniformly dispersed mucus-adherent nanoparticles (polystyrene nanoparticles) in the mucus while maintaining the barrier function of the mucus to herpes simplex virus type-1 (HSV-1). ${ }^{308}$ In support of this result, Xin et al confirmed that cationic surfactant (SDS) and nonionic surfactants (poloxamers [188, 407], polysorbate 80)-pretreated ileum of rats showed enhanced penetration of PLGA nanoparticles into the intestinal epithelium at 30 min of administration, compared to the bare nanoparticles group. ${ }^{309}$ Nanoparticles coated with a nonionic surfactant (poloxamer 407) are known to inhibit the interaction between the core of particles and mucus components. Yang et al prepared surfactant-coated nanoparticles (poloxamer 407-coated fluorescently tagged PLGA nanoparticles) and estimated their penetration in the human mucosa. ${ }^{310}$ The results showed that less than $1 \%$ of bare nanoparticles were dispersed in the mucus layer within 30 min observation time, while $60-80 \%$ of the surfactant-coated nanoparticles were dispersed in the mucus layer. They also reported that this effect was exerted by coating with poloxamer 407, regardless of the type of nanoparticles.

When the nanoparticles pass through the mucus layer, as described above, they reach the small intestine. In the small intestine, three pathways are being reported related to the passage of nanoparticles (Figure 9A-C). (Figure 9A): Transcellular route, which involves the process of transcytosis via intestinal epithelial cells. Particles present in the intestinal lumen are thought to be endocytosed into small intestinal epithelial cells via four different mechanisms: clathrin-mediated endocytosis, caveolamediated endocytosis, micropinocytosis, and phagocytosis. $^{295,311}$ In general, transcytosis via small intestinal epithelial cells has been reported to be easier for particles with smaller size. ${ }^{312,313}$ Rejman et al

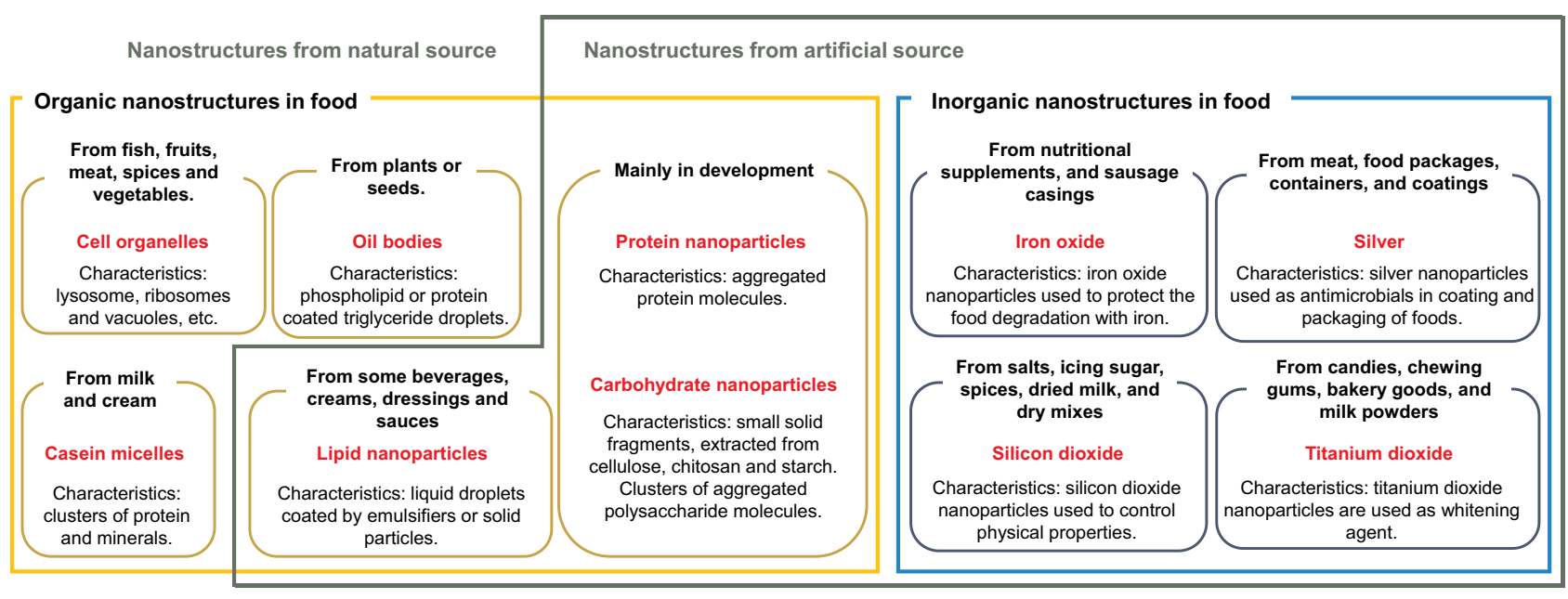

Figure 10 A wide variety of nanoscale materials potentially present in foods from both natural and artificial sources. Notes: Data from McClements and Xiao. ${ }^{291}$ 
demonstrated the uptake mechanism and intracellular accumulation of fluorescent latex beads with a particle size of 50-1000 nm into non-phagocytic B16 melanoma cells. $^{314}$ They found that particles less than $200 \mathrm{~nm}$ in size were taken up into the cells via clathrin-mediated endocytosis, while particles more than $200 \mathrm{~nm}$ in size were taken up into the cells via caveola-mediated endocytosis. There are two routes possible for uptake, one through the $\mathrm{M}$ cells (Figure 9A-1) and the other through the enterocyte (Figure 9A-2). Compared to enterocytes, $\mathrm{M}$ cells are less protected by the mucus barrier and are therefore easier for nanoparticles to reach, but their total area is only about $1 \%$ of the total absorbed surface area of the gut. ${ }^{295,315}$ (Figure 9B): Paracellular route, which involves the transportation through the gaps between small intestinal epithelial cells. The intercellular gap is said to be between 30 and $100 \AA$, with a total area of only $0.1 \%$ of the total absorbed surface area of the gut. ${ }^{316}$ Furthermore, only a few solid nanoparticles are considered to pass through this route, because the majority of nanoparticles are blocked by the presence of tight junctions between epithelial cells. (Figure 9C): Persorption mechanism, which involves a passage through the gap formed in the epithelium by the extrusion of dead intestinal cells from the epithelial layer of the small intestine. ${ }^{317}$ Although the persorption mechanism is considered to allow nanoparticles to pass through in their native state, however due to the small number of research reports, their detailed mechanism is still largely unknown. Nanoparticles are thought to pass through the small intestinal epithelium from either of the above routes and are subsequently transported into the lymph and bloodstream (Figure 9A-C). Lamson et al reported that when negatively charged silica nanoparticles were administered orally to mice, these bound to integrins in small intestinal epithelial cells, induced relaxation of tight junctions, and increased intestinal permeability, which prompted the protein migration further into the bloodstream. ${ }^{318}$ The mechanism involves the binding of negatively charged silica nanoparticles $(<100 \mathrm{~nm})$ to integrins present on epithelial cell surface receptors, thereby stimulating various signaling pathways (Akt, PAK, Raf and Src pathways) that activate the enzyme myosin light chain kinase (MLCK). Activated MLCK phosphorylates the myosin portion of the cytoskeleton and exerts tension on the tight junctions, then leading them to open. The gaps in the tight junctions opened in this way allow the passage of macromolecules without causing cell damage (Figure 9B). Furthermore, they confirmed the effect of insulin (model protein) on blood glucose levels in C57BL/6 mice in which tight junctions were opened by oral administration of above negatively charged silica nanoparticles. The results reported that the orally administered insulin $(10 \mathrm{U} / \mathrm{kg})$ group showed hypoglycemia for several hours longer than the subcutaneously injected insulin $(1 \mathrm{U} / \mathrm{kg})$ group. Other institutions have reported that ultrasound-induced cavitation temporarily weakens the barrier function of the intestinal tract and increases the absorption of drugs (hydrocortisone, insulin, mesalamine) from the small intestine. ${ }^{319}$ A variety of surfactants have been used in the preparation of "smart foods," and many studies on "smart foods" using surfactant-coated nanoparticles have been reported (Table 2). However, there are few reports on the effect of different surfactants on absorption and metabolism in vivo. Although the latter could not be included in this review, future studies are of vital importance for such integrated information.

Along with the uptake of the nanoparticles, their dispersibility in the intestinal lumen before reaching the intestinal mucosa may have a significant impact on the absorption of the food components encapsulated in nanoparticles. Harigae et al prepared PLGA nanoparticles encapsulating curcumin (a polyphenol compound) and compared its oral absorption (area under the curves [AUCs] of curcumin and its main metabolite [curcumin glucuronide]) with the control group of free curcumin in rats. ${ }^{320}$ The results revealed that high concentrations of curcumin glucuronide were present in the blood in case of PLGA nanoparticle-treated group compared to the control group, but the blood concentrations of curcumin were lower in both groups. Furthermore, they confirmed the transport mechanism of curcumin in PLGA nanoparticles to mixed micelles in a human colorectal adenocarcinoma (Caco-2) cell transwell model. As a result, the major factor responsible for the enhanced absorption of curcumin glucuronide in bile acid micelles from PLGA nanoparticles was the high dispersibility of the PLGA nanoparticles in the solution, instead of the pathways described above (Figure 9A-C) or metabolic resistance. It is generally believed that orally administered nutritional components are taken up from the small intestine and transferred to the liver via the portal vein or through the mesenteric lymph nodes, which are subsequently transported to various organs via the lymphoid network and bloodstream. ${ }^{321}$ There are only a few studies available on the behavior of nanoparticles, either metabolism or modification, during their uptake from the small intestine and transfer to the 


\section{Nanoparticles}

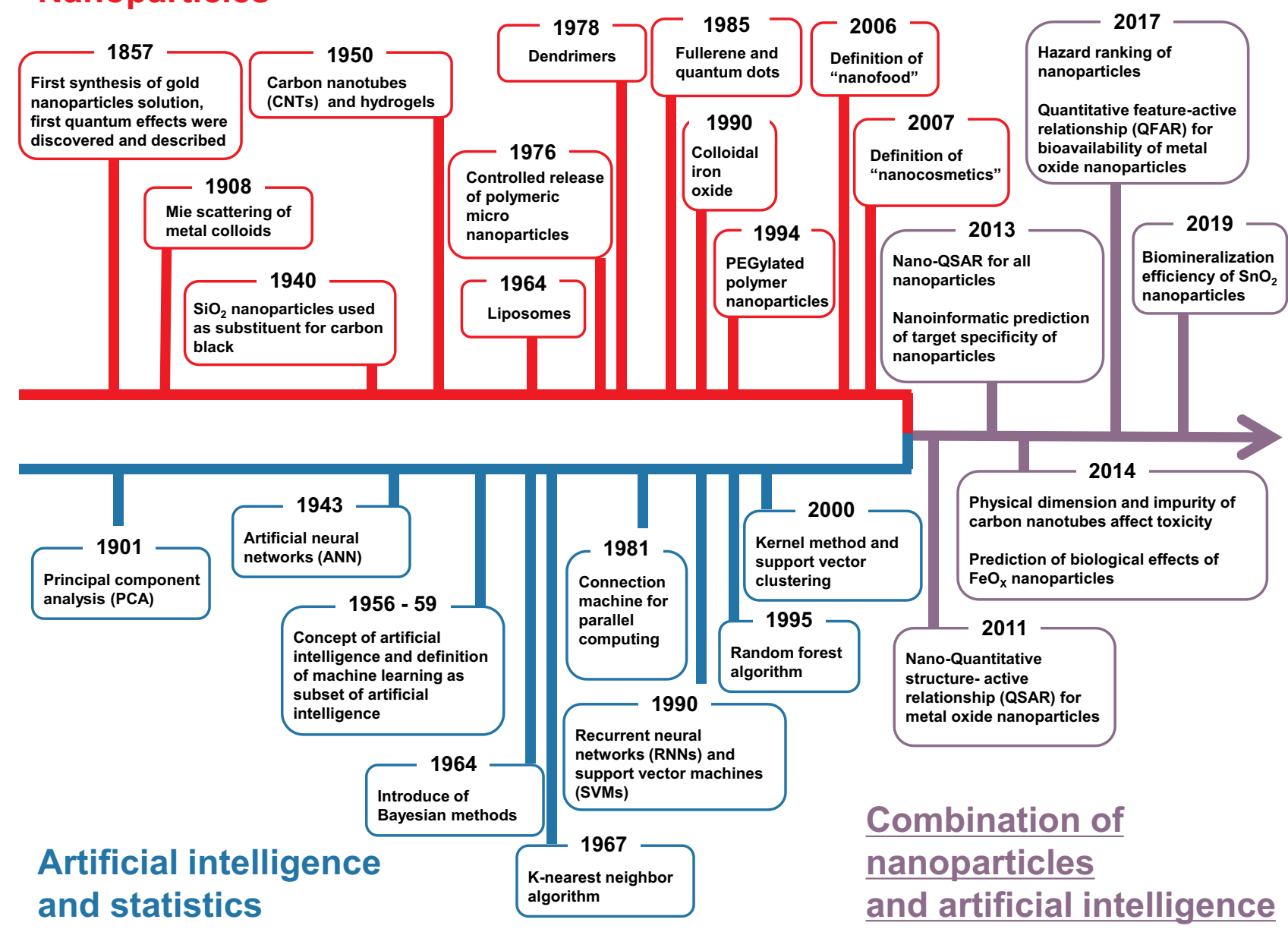

Figure I I Overlapping timelines of the development of artificial intelligence and nanomaterials. Since 20I0, these two fields have developed a powerful synergy. Notes: Modified from Singh AV, Rosenkranz D, Ansari MHD, etal Artificial intelligence and machine learning empower advanced biomedical material design to toxicity prediction. Advanced Intelligent Systems. 2020;2:2000084. ${ }^{379}$

lymph and bloodstream. Itaya et al compared the uptake of curcuminoids-encapsulated PLGA nanoparticles and free curcuminoids into human monocytic leukemia (THP-1) cells. ${ }^{322}$ The results confirmed that only curcumin was selectively taken up among the curcuminoids in both groups, suggesting that curcuminoids are released from PLGA nanoparticles and taken up by the cells after becoming educt. Further investigation of the detailed uptake mechanism revealed that curcuminoids with a higher affinity for albumin (a major transport protein in the blood), were less likely to be taken up by monocytes. Yan et al supported this phenomenon and elucidated that when albumin adheres to the surface of disulfide-stabilized poly (methacrylic acid) nanoporous particles, the nanoparticles are evaded from macrophage uptake. ${ }^{323}$ Future studies are necessary to elucidate the phenomenon of orally administrated nanoparticles in the blood and its further interaction with the blood components through quantitative measurements.

\section{Application of Food Nanotechnology to New Packaging Technologies}

The development of new packaging technologies for food products is important for ensuring food safety. ${ }^{256,257,263}$ The packaging requirements like to allow desirable outside air to pass through depends on the type of foods and beverages to be packaged. For example, carbonated beverage packaging needs to minimize the ingress of oxygen and the loss of carbon dioxide. ${ }^{324}$ In case of fruits and vegetables that require breathing, packaging techniques are required to exchange outside air according to each type. For potatoes, tomatoes and apples, gas transfer of $1-2 \%$ of $\mathrm{O}_{2}, 15-20 \%$ of 
$\mathrm{CO}_{2}$, and $25-30 \%$ of ethylene, respectively is required for their storage. ${ }^{325}$ As a food packaging material, polymeric nanocomposites with organic nanoparticles dispersed in a polymeric matrix can be more precisely manipulated for strength and selective permeability of outside air than the traditional packaging materials used in the past, such as metal, ceramic, paper, and plastic. ${ }^{326}$ Poverenov et al developed alginate nanoparticle-dispersed chitosan polymeric nanocomposites and reported that when coated on fresh-cut melons, it protected against dehydration and ethanol evaporation for 15 days while maintaining selective permeability of the outside air, ideal for storage. ${ }^{327}$ Azeredo et al developed thin films of polymeric nanocomposites containing $15 \%$ cellulose nanofibers and $18 \%$ glycerol as food plasticizers and reported that they exhibited ductility and hydrophobicity while maintaining higher strength than synthetic polymers such as low-density polyethylene and polypropylene. ${ }^{328}$ Furthermore, the film is biodegradable and environmental friendly. Surfactants are used as dispersants for organic nanoparticles in the manufacturing stage of polymeric nanocomposites. ${ }^{329-331}$ Despite the development of polymeric nanocomposites, there are few reports available on the safety of long-term ingestion, ${ }^{332}$ which needs to be considered.

The antimicrobial activity of natural products such as mushrooms in food packaging may help to overcome the food industry challenges of food contamination and spoilage by bacteria. Mushrooms synthesize a variety of metabolites with antitumor, antiviral, anti-inflammatory, antibacterial, antifungal, and anti-yeast activities. ${ }^{333}$ Therefore, there is a growing need for bioprospecting of mushrooms. ${ }^{334}$ And mushrooms are also known to produce multidrug resistance inhibitors that enhance the activity of antimicrobial compounds, and the synergy with silver nanoparticles dramatically improves resistant microorganisms and their antimicrobial activity. This use of mushrooms for application of food nanotechnology to new packaging technologies is thoroughly reviewed in the report by Pandey et al in $2020 .{ }^{334}$ The application of nanotechnology to food packaging to maintain food quality will become even more important in the future. ${ }^{335}$

\section{Surfactant-Coated Inorganic Nanoparticles in Food Nanotechnology}

Food nanosensing, which uses inorganic nanoparticles for sensing of food (adulterant sensing, artificial smell and taste sensing, bacterial toxin sensing, brand protection and product authenticity, freshness sensing, pathogenic bacteria sensing), plays an important role in better food quality and safety evaluation. ${ }^{336}$ Karatapanis et al reported that silica-modified magnetite nanoparticles coated with cationic surfactants can be used as adsorbents for $\mathrm{Cu}(\mathrm{II})$, $\mathrm{Ni}(\mathrm{II}), \mathrm{Co}(\mathrm{II}), \mathrm{Cd}(\mathrm{II}), \mathrm{Pb}(\mathrm{II})$ and $\mathrm{Mn}(\mathrm{II}){ }^{337}$ The detection limits of these elements in aqueous solution were 4.7, 9.1, 9.5, 2.3, 7.4, and $15.3 \mathrm{ng} / \mathrm{L}$, respectively. Zahid et al developed an electrochemical sensor with a surfactant (1(2, 4-dinitrophenyl)-dodecanoylthiourea (DAN), which has soil fertility enhancing characteristics) immobilized at the interface to detect $\mathrm{Hg}$ (II) in drinking water with a detection limit of $0.64 \mu \mathrm{g} / \mathrm{L} .^{338}$ These sensing of food using nanoparticles has been described in detail in other reviews. 339,340

Among the inorganic nanoparticles used in food nanotechnology, silver nanoparticles are the most widely used in the food industry due to their antimicrobial properties. Approximately $55.4 \%$ of all consumer products using nanoparticles in the market are made with silver nanoparticles. ${ }^{341}$ In addition, several countries are already using silver nanoparticles as antimicrobial agents in food supplements and food packaging materials. ${ }^{342}$ When silver nanoparticles reach the bacterial cell surface, they form irregularly shaped pits on the membrane surface, reducing the barrier function of lipopolysaccharides present on the cell surface, and thereby altering the membrane permeability. ${ }^{343}$ Subsequently, silver cation $\left(\mathrm{Ag}^{+}\right)$is generated by protons and enzymes present in the bacterial cell, causing an increase in oxidative stress due to ROS and inhibition of deoxyribonucleic acid (DNA) replication, leading to bacterial cell death. ${ }^{344,345}$ Costa et al prepared an alginate film containing silver nanoparticles and reported that coating it on fresh-cut carrots protected the carrots from dehydration and microbial spoilage and extended the shelf life from four days (in non-additive group) to 70 days. $^{346}$ Hedayati et al prepared surfactantcoated nanoparticles (gum Arabic containing silver nanoparticles and a nonionic surfactant (glyceryl monostearate [Figure 1]). ${ }^{347}$ They reported that coating green bell pepper with this product protected the antioxidant (vitamin C) in the green bell pepper from dehydration and microbial spoilage and maintained marketable quality even after 21-days of storage. Since silver nanoparticles need to be in a dispersed state to exert their antibacterial effect, the approach of coating their surface with a non-ionic surfactant and dispersing them in a system is widely used. Kvitek et al prepared surfactant-coated nanoparticles 
(silver nanoparticles coated with SDS or polysorbate 80) and confirmed their antimicrobial activity. ${ }^{348}$ Results showed that coating with both surfactants had increased antimicrobial activity of silver against 10 strains of bacteria compared to bare nanoparticles, and the SDS-coated nanoparticles exhibited more potency. In addition to silver nanoparticles, zinc oxide nanoparticles are used as supplements, antibacterial agents, and anti-browning agents. ${ }^{349}$ $\mathrm{Li}$ et al reported the use of zinc oxide nanoparticles as an anti-browning agent for food products. ${ }^{350}$ They developed a polyvinyl chloride nanoparticles containing zinc oxide $(\mathrm{ZnO})$, and when Fuji apples were coated with it, the activity of polyphenol oxidase and pyrogallol peroxidase was suppressed, resulting in reduced formation of malondialdehyde (reduced from $74.9 \mathrm{nmol} / \mathrm{g}$ (untreated group) to $53.9 \mathrm{nmol} / \mathrm{g}$ ) and decrease in the browning index (reduced from 31.7 (untreated group) to 23.9), after 12-days. Several studies on the use of silver nanoparticles and their potential application for antiviral effects have also been reported. Huy et al reported that silver nanoparticles were not toxic to normal cells (human rhabdomyosarcoma cell line), while they exhibited toxicity to a non-enveloped virus (poliovirus) in vitro. ${ }^{351}$ Sreekanth et al also reported that the preparation of silver nanoparticles using terpenoid and flavonoid mixtures extracted from the roots of ginseng by green synthesis and showed toxicity to the influenza $\mathrm{A}$ virus, while they did not exhibit toxicity to assumed normal cells (Madin-Darby canine kidney [MDCK] cell line). ${ }^{352}$ Antoine et al reported that zinc oxide nanoparticles greatly inhibited herpes simplex virus type 2 (HSV-2) infection of the reproductive organs in female BALB/c mice and reduced mortality. ${ }^{353}$ Gurunathan et al reviewed in 2020 the possibility that antiviral potential of inorganic nanoparticles might be a fight against coronaviruses. ${ }^{354}$ Other inorganic nanoparticles such as those containing iron oxide (supplements and colorants), titanium dioxide (food additives), silica (anti-caking agents and flavors), and selenium (supplements) are used in food nanotechnology. ${ }^{290,355-357}$ It is believed that surfactants can also be used for these inorganic nanoparticles other than silver nanoparticles to further enhance their functions in the future. On the contrary, Gram-negative bacteria such as Escherichia coli 013, Pseudomonas aeruginosa CCM3955, and Escherichia coli CCM3954 gradually acquire resistance to inorganic nanoparticles. ${ }^{358}$ In addition, silver, titanium dioxide, zinc oxide, and silica nanoparticles reach the colon after oral administration, and their antimicrobial properties can affect the intestinal microbiota and aggravate the immune response of the gut-associated lymphoid tissue. ${ }^{359}$ New technologies in food nanotechnology are expected to overcome these current concerns.

\section{Nanoparticles Originally Contained in Food}

Humans consume food products containing nanoscale substances on daily basis. There are numerous nanostructures (such as emulsion, nanoparticles and micelles, and colloids) composed of proteins, carbohydrates, and lipids that exist in the food matrix. For example, milk contains nanostructures such as casein micelles (50-300 nm in diameter), whey protein (4-6 nm in diameter), and lactose $(0.5 \mathrm{~nm}$ in diameter). A wide variety of nanoscale materials, from both natural and artificial sources, might be present in foods (Figure 10). ${ }^{291}$ Several studies have been conducted on these nanostructures present in food; beer (containing microplastics), ${ }^{360}$ chewing gum (containing titanium dioxide), ${ }^{361}$ chicken meat (containing silver nanoparticles),362 drink products (containing silver, gold, copper, iridium, palladium, platinum, silicon, and zinc nanomaterials), ${ }^{363}$ drinking water (containing titanium dioxide, silver, and gold nanoparticles), ${ }^{364}$ and honey (containing non-pollen particles). ${ }^{365}$ Zhang et al used atomic force microscopy to examine the nanostructure of pectin in cherries and found a close relationship between its structure and fruit firmness. ${ }^{366}$ Dang et al also reported that cooking and processing of foods can change their nanostructures, which further changes the physical properties of the foods. ${ }^{367}$ It has been reported that ferritin nanoparticles contained in plant-based foods are taken up from small intestinal epithelial cells and used as a source of iron in the body. ${ }^{368}$ Nanostructures are also produced during oral ingestion, for example, orally ingested foods can be physically (emulsification, mastication, and peristalsis) and chemically (acidic $\mathrm{pH}$ environment and interaction with various digestive enzymes) stimulated in various organs of the digestive system, some of which are miniaturized to the nanoscale. It is believed that the food components (amino acids, inorganic salts, monosaccharides, polyphenols, and vitamins) are miniaturized in this way and reaches the small intestine, where they are subsequently absorbed. ${ }^{241,321}$ Some nanoparticles are formed in the digestive organs by chemical precipitation. For example, when food-derived calcium and phosphate ions are present in the small intestine, calcium phosphate nanoparticles are deposited. ${ }^{369}$ Thus, nanoparticles are present in many food products, however, 
the "nanostructures that are not purpose-designed and are originally contained in food", as described in this section, are not considered as the products of food nanotechnology. ${ }^{265}$

\section{Safety of Nanoparticles in the Food Industry}

The safety of nanoparticles in food industry is an important concern that cannot be ignored. Many studies have claimed that nanoparticles may migrate from packaging materials into food, then taken up and accumulated in the human body via oral intake. ${ }^{370}$ In addition, nanoscale pesticide residues present in food and other foreign substances that have contaminated the food products during their manufacturing process are unintentional contaminants. Distinguishing such contaminants from the nanoparticles prepared by food nanotechnology or nanoparticles originally contained in food or food-derived nanoparticles is important for food quality, safety, and environmental considerations. Inorganic nanoparticles that may be contaminated in the food include transition metals (for example, silver, iron, titanium, and zinc), alkali earth metals (calcium and magnesium), and non-metals (selenium and silicate). In developed countries, it is estimated that more than $10^{12}$ inorganic nanoparticles are taken into the human body per day. ${ }^{371}$ Some nanoparticles are also unintentionally contaminated in animal and plant breeding environments. For example, Lin et al examined the transfer and accumulation of two types of nanostructures (C70 fullerene and multiwalled carbon nanotubes) into plants through their growing environment. ${ }^{372}$ Rice seedlings were grown in a germination culture medium containing C70 fullerene or multiwalled carbon nanotubes for two weeks. Then, they were transplanted into the soil and grown to maturity. Rice seeds were taken from the first generation of plants grown in this manner, and the second generation was grown in a nanoparticle-free germination buffer. They found that C70 fullerenes were present in the first generation of seeds grown in an environment containing nanoparticles for a long period. The presence of $\mathrm{C} 70$ fullerene was also observed in the leaves of the second generation grown from the $\mathrm{C} 70$ fullerene-accumulating seeds obtained in the first generation. In contrast, the plants grown in the germination culture medium containing multi-walled carbon nanotubes did not show their accumulation in either first or second generation. This suggests that C70 fullerene and multiwalled carbon nanotubes accumulate differently in the plant body depending on their nanostructures. Such uptake of nanoparticles into plants has also been studied with metal nanoparticles such as gold, silver and silica. ${ }^{373-375}$ The nanostructures have the potential risk of exposure and accumulation through various routes in the human body, such as unintentional inhalation and dermal contact. ${ }^{376}$ At this stage, the types and amounts of nanostructures present in the environment, their accumulation in plants and animals, and the risk of pollution in the food supply are not yet well understood, which is very important considering the use of nanoparticles in food industry. Bieberstein et al assessed consumer inclination towards purchase of products of food nanotechnology in France and Germany, focusing on two applications: "nano vitamin" and "nano packaging." The results reported that consumers in both countries tended to be reluctant to accept food nanotechnology. ${ }^{377}$ These trends are due to unresolved concerns about the safety of products of food nanotechnology, which needs to be assessed through research that is more extensive.

\section{Conclusions and Outlook}

Surfactants have a long history of use by humans, and various products have been made that make use of their properties. Moreover, the surfactant-coated nanoparticles demonstrated the crucial importance of surfactants in the fields of nanomedicine and food nanotechnology. In both fields, it has been demonstrated that surfactants can further enhance the functions of the nanoparticles. Various approaches that take advantage of the synergistic effects of nanoparticles and surfactants have the potential to create many useful new technologies in the future, although challenges are yet to be overcome, including safety of nanoparticles and surfactants. There are many cases where technologies that are highly effective in vitro do not work well in vivo because of unexpected problems that arise when applied to humans. While new technologies are being developed everyday, the rapid development of nanomedicine and food nanotechnology has also ignited consumer concerns. Current information on nanoparticles and surfactants is insufficient to overcome these concerns. Therefore, continuing research is needed to obtain reliable information in the future. Recently, advances in machine learning and artificial intelligence immensely decoded and 
empowered, the cell-nanomaterial interaction modelling, which gave modern to nanomedicine to predict the biosafety and efficacy ${ }^{378,379}$ and in-silico methods ${ }^{380,381}$ to potentially decipher the quantitative nanostructure activity-relationship (Nano-QSAR). In 2010, the two timelines (nanoparticles and artificial intelligence) merged as artificial intelligence was applied to the task of identifying and predicting of grouping according to their properties, interaction, and toxicity of nanoparticles (Figure 11). ${ }^{379}$ The fields of nanoparticles and artificial intelligence will continue to complement each other. There will be significant progress in research field of surfactant-coated nanoparticles as the develop of these technologies. This review is not limited to either nanomedicine or food nanotechnology, but is intended to be of interest to people in both fields and to bridge these fields. We hope that this review will serve as an impetus for the development of new technologies in interdisciplinary fields.

\section{Abbreviations}

ABC, accelerated blood clearance; AOT, sodium bis(2-ethylhexyl) sulfosuccinate; ATP, adenosine triphosphate; AUCs, area under the curves; BBB, blood-brain barrier; BDM, Bockris/Devanathan/Muller; Brij 35, polyoxyethylene lauryl ether; BSA, bovine serum albumin; CAGR, compound annual growth rate; $\mathrm{CPP}$, critical packing parameter; $\mathrm{CTAB}$, cetyltrimethylammonium bromide; DAN, 1-(2, 4-dinitrophenyl)-dodecanoylthiourea; DDAB, didodecyldimethylammonium bromide; DLT, dose-limiting toxicity; DLVO, Derjaguin-Landau-Verwey-Overbeek; DMEM, Dulbecco's Modified Eagles Medium; DNA, deoxyribonucleic acid; DOTAP, 1， 2-dioleoyl-3-trimethylammonium-propane; EGFR mAb, epidermal growth factor receptor monoclonal antibodies; EPR, enhanced permeability and retention; FBS, fetal bovine serum; FDA, Food and Drug Administration; GLUT, glucose transporter; GPX, glutathione peroxidase; HLB, hydrophilic-hydrophobic balance; HIV-1, human immunodeficiency virus type 1; HSV-1, herpes simplex virus type-1; HSV-2, herpes simplex virus type 2; HUVECs, human umbilical vein endothelial cells; $\mathrm{KC} /$ GRO, keratinocyte chemoattractant / growth-regulated oncogene chemokines; M1, classically activated; MPS, mononuclear phagocyte system; IgG, immunoglobulin G; IL, interleukin; MDCK, Madin-Darby canine kidney; MLCK, myosin light chain kinase; MR, magnetic resonance; MRI, magnetic resonance imaging; NADPH, nicotinamide adenine dinucleotide phosphate; Nano-QSAR, quantitative nanostructure activity-relationship; OAPB, oleyl amidopropyl betaine; OECD, Organization for Economic Cooperation and Development; O/W, oil in water; PBCA, poly (butyl cyanoacrylate); $\mathrm{PBOB}$, perfluorooctyl bromide; PEG, polyethylene glycol; PEO, polyoxyethylene oxide; PPO, polypropylene oxide; Pgp/ABCB1, P-glycoprotein; PLA, polylactic acid; PLGA, poly(Poly lactic-co-glycolic acid); PVA, polyvinyl alcohol; poloxamer 188, poly(ethylene glycol)-block-poly(propylene glycol)-block-poly(ethylene glycol); polysorbate 80, polyoxyethylene (20) sorbitan monooleate; RBCs, red blood cells; RES, reticuloendothelial system; RF, radiofrequency; rhEPO, recombinant human erythropoietin; ROS, reactive oxygen species; SB3-14, 3[N,N-dimethyltetradecylammonio]propane-1-sulfonate; SC, sodium cholate; SD, sodium deoxycholate monohydrate; SDS, sodium dodecyl sulfate; SLS, sodium lauryl sulfate; siRNA, small interfering RNA; SOD, superoxide dismutase; span85, sorbitan trioleate; SPION, superparamagnetic iron oxide nanoparticles; SPR, surface plasmon resonance; TC, sodium taurocholate; TEM, transmission electron microscopy; Th1, type 1 helper; TNF- $\alpha$, tumor necrosis factor.

\section{Acknowledgments}

This review could never have been completed without the contribution of many researchers from the past to the present in the fields of surfactants, nanomedicines, and food nanotechnology. We thank all the researchers who made this review possible. This work was supported in part by the JSPS KAKENHI Fellowship for Young Scientists (17J09342, 19J21566) and JSPS KAKENHI Grant-in-Aid for Young Scientists (18K14419). We would like to thank Editage for English language editing.

\section{Disclosure}

The authors report no conflicts of interest in this work.

\section{References}

1. Dutta AK. Introductory chapter: surfactants in household and personal care formulations - An overview. IntechOpen. 2019. doi:10.5772/ intechopen. 89245

2. Hill M, Moaddel T. 2 - Soap structure and phase behavior. Soap Manufacturing Tech. 2016;35-54. doi:10.1016/B978-1-63067-0658.50002-5

3. Effendy I, Maibach HI. Surfactants and experimental irritant contact dermatitis. Contact Dermatitis. 1995;33:217-225. doi:10.1111/j.16000536.1995.tb00470.x

4. Lottermoser A, Stoll F. Untersuchungen über die oberflächen- und grenzflächenaktivität von salzen der fettalkoholschwefelsäureester. Kolloid-Zeitschrift. 1933;63:49-61. doi:10.1007/BF01427993

5. Kogawa AC, Cernic BG, Do Couto LGD, et al. Synthetic detergents: 100 years of history. Saudi Pharm J. 2017;25:934-938. doi:10.1016/j. jsps.2017.02.006 
6. Pettersson A, Adamsson M, Dave G. Toxicity and detoxification of Swedish detergents and softener products. Chemosphere. 2000;41:1611-1620. doi:10.1016/S0045-6535(00)00035-7

7. Liu B, Li T, Wang W, et al. Corncob cellulose nanosphere as an eco-friendly detergent. Nat Sustain. 2020;3:448-458. doi:10.1038/s41893-020-0501-1

8. Wood L $\$ 64.4$ Billion surfactants market by type and application Global opportunity analysis \& industry forecast (2018-2025). Available from: ResearchAndMarkets.com. Accessed April 19, 2021.

9. Taniguchi N. On the basic concept of nanotechnology. Proce ICPE. 1974.

10. Feynman RP. There's plenty of room at the bottom. Resonance. 2011;16(9):890. doi:10.1007/s12045-011-0109-x

11. Kumar D, Rub MA. Interaction of metal ion-coordinated dipeptide complex and ninhydrin in the alkanediyl- $\alpha, \omega$-bis-type gemin surfactant system. J Surfactants Deterg. 2019;22(6):1299-1308. doi:10.1002/jsde. 12340

12. Schramm LL, Stasiuk EN, Marangoni DG. 2 Surfactants and their applications. Ann Rep Section C. 2003;99:3-48. doi:10.1039/ B208499F

13. McCoy TM, Marlow JB, Armstrong AJ, et al. Spontaneous selfassembly of thermoresponsive vesicles using a zwitterionic and an anionic surfactant. Biomacromolecules. 2020;21(11):45694576. doi:10.1021/acs.biomac.0c00672

14. Puri C, Pucciarini L, Tiecco M, et al. Use of a zwitterionic surfactant to improve the biofunctional properties of wool dyed with an onion (Allium cepa L.) Skin Extract. Antioxidants. 2020;9 (11):1055. doi:10.3390/antiox9111055

15. Vlachy N, Touraud D, Heilmann J, et al. Determining the cytotoxicity of catanionic surfactant mixtures on HeLa cells. Colloids Surf $B \quad$ Biointerfaces. 2009;70(2):278-280. doi:10.1016/j. colsurfb.2008.12.038

16. Berardesca E, Fideli D, Gabba P, et al. Ranking of surfactant skin irritancy in vivo in man using the plastic occlusion stress test (POST). Contact Dermatitis. 1990;23(1):1-5. doi:10.1111/j.16000536.1990.tb00075.x

17. Korting HC, Herzinger T, Hartinger A, et al. Discrimination of the irritancy potential of surfactants in vitro by two cytotoxicity assays using normal human keratinocytes, HaCaT cells and 3T3 mouse fibroblasts: correlation with in vivo data from a soap chamber assay. $J$ Dermatol Sci. 1994;7(2):119-129. doi:10.1016/0923-1811(94)90085-X

18. Grant RL, Yao C, Gabaldon D, et al. Evaluation of surfactant cytotoxicity potential by primary cultures of ocular tissues: I. Characterization of rabbit corneal epithelial cells and initial injury and delayed toxicity studies. Toxicology. 1992;76(2):153-176. doi:10.1016/0300-483X(92)90162-8

19. Mullerdecker K, Furstenberger G, Marks F. Keratinocyte-derived proinflammatory key mediators and cell viability as in vitro parameters of irritancy: a possible alternative to the draize skin Irritation Test. Toxicol Appl Pharmacol. 1994;127(1):99-108. doi:10.1006/taap.1994.1144

20. Karim KM, Mandal AS, Biswas N et al. Niosome: a future of targeted drug delivery systems. J Adv Pharm Technol Res. 2010;1 (4):374-380. doi:10.4103/0110-5558.76435

21. Beneito-Cambra M, Herrero-Martínez JM, Ramis-Ramos G. Analytical methods for the characterization and determination of nonionic surfactants in cosmetics and environmental matrices. Anal. Methods. 2013;5(2):341-354. doi:10.1039/C2AY25847A

22. Rosen MJ, Kunjappu JT. Characteristic features of surfactants. Surfactants Interfacial Phenomena. 2012;1-38. doi:10.1002/ 9781118228920.ch1

23. Lombardo D, Kiselev MA, Magazù S, et al. Amphiphiles selfassembly: basic concepts and future perspectives of supramolecular approaches. Adv Condensed Matter Phys. 2015;2015:1-22. doi:10.1155/2015/151683
24. Foo KS, Bavoh CB, Lal B, et al. Rheology impact of various hydrophilic-hydrophobic balance (HLB) index non-ionic surfactants on cyclopentane hydrates. Molecules. 2020;25(16):3725. doi:10.3390/molecules25163725

25. Nagarajan R. Molecular Packing Parameter and Surfactant SelfAssembly: the Neglected Role of the Surfactant Tail $\uparrow$. Langmuir. 2002;18(1):31-38. doi:10.1021/la010831y

26. Kunieda H, Shinoda K. Evaluation of the hydrophile-lipophile balance (HLB) of nonionic surfactants. I. Multisurfactant systems. $J$ Colloid Interface Sci. 1985;107(1):107-121. doi:10.1016/0021-9797(85)90154-7

27. Singh AV, Hosseinidoust Z, Park B, et al. Microemulsion-based soft bacteria-driven microswimmers for active cargo delivery. ACS Nano. 2017;11(10):9759-9769. doi:10.1021/acsnano.7b02082

28. Miyazawa T, Matsuda K, Fujimori A, et al. One-pot synthesis of manganese oxide nanoparticles from microemulsion systems. Chem Lett. 2011;40(11):1262-1263. doi:10.1246/cl.2011.1262

29. Tian Z, Tong W, Wang J, et al. Manganese oxide mesoporous structures: mixed-valent semiconducting catalysts. Science. 1997;276:926-930. doi:10.1126/science.276.5314.926

30. Kubacka A, Caudillo-Flores U, Barba-Nieto $I$, et al. Microemulsion: a versatile synthesis tool for photocatalysis. Curr Opin Colloid Interface Sci. 2020;49:42-59. doi:10.1016/j. cocis.2020.04.009

31. Thiele MJ, Davari MD, Hofmann I, et al. Enzyme-compatible dynamic nanoreactors from electrostatically bridged like-charged surfactants and polyelectrolytes. Angewandte Chemie Int Edition. 2018;57:9402-9407. doi:10.1002/anie.201805021

32. Boonme P. Applications of microemulsions in cosmetics. $J$ Cosmet Dermatol. 2007;6:223-228. doi:10.1111/j.14732165.2007.00337.x

33. Qin T, Goual L, Piri M, et al. Nanoparticle-stabilized microemulsions for enhanced oil recovery from heterogeneous rocks. Fuel. 2020;274:117830. doi:10.1016/j.fuel.2020.117830

34. Mashaghi S, Jadidi T, Koenderink G, et al. Lipid nanotechnology. Int J Mol Sci. 2013;14:4242-4282. doi:10.3390/ijms14024242

35. Ge X, Wei M, He S, et al. Advances of non-ionic surfactant vesicles (niosomes) and their application in drug delivery. Pharmaceutics. 2019;11:55. doi:10.3390/pharmaceutics11020055

36. Bartelds R, Nematollahi MH, Pols T, et al. Niosomes, an alternative for liposomal delivery. PLoS One. 2018;13:e194179. doi:10.1371/journal.pone.0194179

37. Puras G, Mashal M, Zárate J, et al. A novel cationic niosome formulation for gene delivery to the retina. J Controlled Release. 2014;174:27-36. doi:10.1016/j.jconrel.2013.11.004

38. Wang W. Protein aggregation and its inhibition in biopharmaceutics. Int J Pharm. 2005;289:1-30. doi:10.1016/j.ijpharm.2004.11.014

39. Katz JS, Nolin A, Yezer BA, et al. Dynamic properties of novel excipient suggest mechanism for improved performance in liquid stabilization of protein biologics. Mol Pharm. 2019;16:282-291. doi:10.1021/acs.molpharmaceut.8b00984

40. Li J, Pinnamaneni S, Quan Y, et al. Mechanistic understanding of protein-silicone oil interactions. Pharm Res. 2012;29:1689-1697. doi:10.1007/s11095-012-0696-6

41. Arsiccio A, McCarty J, Pisano R, et al. Effect of surfactants on surface-induced denaturation of proteins: evidence of an orientation-dependent mechanism. J Phys Chem B. 2018;122:1139011399. doi:10.1021/acs.jpcb.8b07368

42. Doshi N, Demeule B, Yadav S. Understanding particle formation: solubility of free fatty acids as polysorbate 20 degradation byproducts in therapeutic monoclonal antibody formulations. Mol Pharm. 2015;12:3792-3804. doi:10.1021/acs.molpharmaceut.5b00310

43. Gervasi V, Dall Agnol R, Cullen S, et al. Parenteral protein formulations: an overview of approved products within the european union. Eur J Pharm Biopharmaceutics. 2018;131:8-24. doi:10.1016/j.ejpb.2018.07.011 
44. Van Damme L, Ramjee G, Alary M, et al. Effectiveness of COL1492, a nonoxynol-9 vaginal gel, on HIV-1 transmission in female sex workers: a randomised controlled trial. Lancet. 2002;360:971-977. doi:10.1016/s0140-6736(02)11079-8

45. Wilkinson D. Nonoxynol-9 fails to prevents STDs, but microbicide research continues. Lancet. 2002;360:962-963. doi:10.1016/ S0140-6736(02)11119-6

46. Naik MK, Naik SN, Mohanty S. Enzymatic glycerolysis for conversion of sunflower oil to food based emulsifiers. Catalysis Today. 2014;237:145-149. doi:10.1016/j.cattod.2013.11.005

47. Jackman JA, Boyd RD, Elrod CC. Medium-chain fatty acids and monoglycerides as feed additives for pig production: towards gut health improvement and feed pathogen mitigation. J Anim Sci Biotechnol. 2020;11:44. doi:10.1186/s40104-020-00446-1

48. El-Adawy TA, Taha KM. Characteristics and composition of watermelon, pumpkin, and paprika seed oils and flours. J Agric Food Chem. 2001;49:1253-1259. doi:10.1021/jf001117+

49. Dima C, Assadpour E, Dima S, et al. Bioavailability of nutraceuticals: role of the food matrix, processing conditions, the gastrointestinal tract, and nanodelivery systems. Comprehen Rev Food Sci Food Safety. 2020;19:954-994. doi:10.1111/ 1541-4337.12547

50. Salvia-Trujillo L, Qian C, Martín-Belloso O, et al. Influence of particle size on lipid digestion and $\beta$-carotene bioaccessibility in emulsions and nanoemulsions. Food Chem. 2013;141:1472-1480. doi:10.1016/j.foodchem.2013.03.050

51. Lu W, Kelly AL, Miao S. Improved bioavailability of encapsulated bioactive nutrients delivered through monoglyceride-structured O/W emulsions. J Agric Food Chem. 2017;65:3048-3055. doi:10.1021/acs.jafc.6b05644

52. Lee J, Kim Y, Shin D. Antimicrobial synergistic effect of linolenic acid and monoglyceride against bacillus cereus and staphylococcus aureus. J Agric Food Chem. 2002;50:2193-2199. doi:10.1021/jf011175a

53. Yaghmur A, Al-Hosayni S, Amenitsch H, et al. Structural investigation of bulk and dispersed inverse lyotropic hexagonal liquid crystalline phases of eicosapentaenoic acid monoglyceride. Langmuir. 2017;33:14045-14057. doi:10.1021/acs.langmuir.7b0 3078

54. Khaddaj-Mallat R, Morin C, Rousseau É. Novel n-3 PUFA monoacylglycerides of pharmacological and medicinal interest: antiinflammatory and anti-proliferative effects. Eur $J$ Pharmacol. 2016;792:70-77. doi:10.1016/j.ejphar.2016.10.038

55. Kralova I, Sjöblom J. Surfactants used in food industry: a review. J Dispers Sci Technol. 2009;30:1363-1383. doi:10.1080/019326 90902735561

56. Aswathanarayan JB, Vittal RR. Nanoemulsions and their potential applications in food industry. Front Sustainable Food Sys. 2019;3. doi: $10.3389 /$ fsufs. 2019.00095

57. Singh C, Ghorai PK, Horsch MA, et al. Entropy-mediated patterning of surfactant-coated nanoparticles and surfaces. Phys Rev Lett. 2007;99:226106. doi:10.1103/PhysRevLett.99.226106

58. Cardellini A, Alberghini M, Govind Rajan A, et al. Multi-scale approach for modeling stability, aggregation, and network formation of nanoparticles suspended in aqueous solutions. Nanoscale. 2019;11:3979-3992. doi:10.1039/C8NR08782B

59. Stiles PJ. Dipolar van der Waals forces between molecules of ellipsoidal shape. Mol Phys. 1979;38:433-447. doi:10.1080/ 00268977900101791

60. Israelachvili JN. Chapter 13 - Van der Waals forces between particles and surfaces. Intermol Surface Forces. 2011;253-289. doi:10.1016/B978-0-12-391927-4.10013-1

61. Chen J, Lee MK, Qin E, et al. Van der Waals force-induced loading of proangiogenic nanoparticles on microbubbles for enhanced neovascularization. Nanoscale. 2015;7:17139-17147. doi: $10.1039 / \mathrm{c} 5 \mathrm{nr} 03399 \mathrm{c}$
62. Wijenayaka LA, Ivanov MR, Cheatum CM, et al. Improved parametrization for extended Derjaguin, Landau, Verwey, and Overbeek predictions of functionalized gold nanosphere stability. J Phys Chem C. 2015;119:10064-10075. doi:10.1021/acs.jpcc.5b00483

63. Hamley IW. Chapter-3 Colloids. Introduction to Soft Matter: synthetic and Biological Self-Assembling Materials. 2007:111159. doi::10.1002/9780470517338.ch3

64. Endo M, Takeda T, Kim YJ, et al. High power electric double layer capacitor (EDLC's); from operating principle to pore size control in advanced activated carbons. Carbon Letters. 2001;1:117-128.

65. Ohshima H. Approximate analytic expression for the stability ratio of colloidal dispersions. Colloid Polym Sci. 2014;292:2269-2274. doi:10.1007/s00396-014-3257-1

66. Ninham BW. On progress in forces since the DLVO theory. $A d v$ Colloid Interface Sci. 1999;83:1-17. doi:10.1016/S0001-8686(99) 00008-1

67. McGown DNL, Parfitt GD. Stability of non-aqueous dispersions. Kolloid-Zeitschrift und Zeitschrift für Polymere. 1967;219:48-51. doi:10.1007/BF02086095

68. Manohar C, Kelkar VK, Yakhmi JV. Electroflotation of colloids without surfactants. J Colloid Interface Sci. 1982;89:54-60. doi:10.1016/0021-9797(82)90119-9

69. Farrokhbin M, Stojimirović B, Galli M, et al. Surfactant mediated particle aggregation in nonpolar solvents. Phys Chem Chem Phys. 2019;21:18866-18876. doi:10.1039/C9CP01985E

70. Espinosa CE, Guo Q, Singh V, et al. Particle charging and charge screening in nonpolar dispersions with nonionic surfactants. Langmuir. 2010;26:16941-16948. doi:10.1021/la1033965

71. Boström M, Williams DRM, Ninham BW. Specific Ion effects: why DLVO theory fails for biology and colloid systems. Phys Rev Lett. 2001;87:168103. doi:10.1103/PhysRevLett.87.168103

72. Xiang L, Zhang J, Gong L, et al. Surface forces and interaction mechanisms of soft thin films under confinement: a short review. Soft Matter. 2020;16:6697-6719. doi:10.1039/D0SM00924E

73. Voorhees PW. The theory of Ostwald ripening. J Stat Phys. 1985;38:231-252. doi:10.1007/BF01017860

74. Anton N, Benoit J-P, Saulnier P. Design and production of nanoparticles formulated from nano-emulsion templates-A review. $J$ Controlled Release. 2008;128:185-199. doi:10.1016/j. jconrel.2008.02.007

75. Kiss E, Bertoti I, Vargha-Butler EI. XPS and Wettability Characterization of Modified Poly(lactic acid) and Poly(lactic/ glycolic acid) Films. J Colloid Interface Sci. 2002;245:91-98. doi:10.1016/j.jconrel.2008.02.007

76. Moore TL, Rodriguez-Lorenzo L, Hirsch V, et al. Nanoparticle colloidal stability in cell culture media and impact on cellular interactions. Chem Soc Rev. 2015;44:6287-6305. doi:10.1039/ C4CS00487F

77. Santander-Ortega MJ, Jódar-Reyes AB, Csaba N, et al. Colloidal stability of Pluronic F68-coated PLGA nanoparticles: a variety of stabilisation mechanisms. J Colloid Interface Sci. 2006;302:522529. doi:10.1016/j.jcis.2006.07.031

78. Lakshman JP, Cao Y, Kowalski J, et al. Application of melt extrusion in the development of a physically and chemically stable high-energy amorphous solid dispersion of a poorly water-soluble drug. Mol Pharm. 2008;5:994-1002. doi:10.1021/ mp8001073

79. Dave RH, Patel HH, Donahue E, et al. To evaluate the change in release from solid dispersion using sodium lauryl sulfate and model drug sulfathiazole. Drug Dev Ind Pharm. 2013;39:15621572. doi:10.3109/03639045.2012.725731

80. Dave RH, Patel AD, Donahue E, et al. To evaluate the effect of addition of an anionic surfactant on solid dispersion using model drug indomethacin. Drug Dev Ind Pharm. 2012;38:930-939. doi:10.3109/03639045.2011.633264 
81. Chaudhari SP, Dugar RP. Application of surfactants in solid dispersion technology for improving solubility of poorly water soluble drugs. J Drug Deliv Sci Technol. 2017;41:68-77. doi:10.1016/j.jddst.2017.06.010

82. Kim BYS, Rutka JT, Chan WCW. Nanomedicine. $N$ Eng J Med. 2010;363:2434-2443. doi:10.1056/NEJMra0912273

83. Etheridge ML, Campbell SA, Erdman AG, et al. The big picture on nanomedicine: the state of investigational and approved nanomedicine products. Nanomedicine. 2013;9:1-14. doi:10.1016/j. nano.2012.05.013

84. Rademaker-Lakhai JM, Crul M, Zuur L, et al. Relationship between cisplatin administration and the development of ototoxicity. J Clin Oncol. 2006;24:918-924. doi:10.1200/jco.2006.10.077

85. Li S-D, Huang L. Pharmacokinetics and biodistribution of nanoparticles. Mol Pharm. 2008;5:496-504. doi:10.1021/mp800049w

86. Jahangirian H, Lemraski EG, Webster TJ, et al. A review of drug delivery systems based on nanotechnology and green chemistry: green nanomedicine. Int J Nanomedicine. 2017;12:2957-2978. doi:10.2147/ijn.S127683

87. Almeida AJ, Souto E. Solid lipid nanoparticles as a drug delivery system for peptides and proteins. Adv Drug Deliv Rev. 2007;59:478-490. doi:10.1016/j.addr.2007.04.007

88. Liu D, Yang F, Xiong F, et al. The smart drug delivery system and its clinical potential. Theranostics. 2016;6:1306-1323. doi:10.7150/thno. 14858

89. Kumari A, Yadav SK, Yadav SC. Biodegradable polymeric nanoparticles based drug delivery systems. Colloids Surf B Biointerfaces. 2010;75:1-18. doi:10.1016/j.colsurfb.2009.09.001

90. Mukherjee S, Ray S, Thakur RS. Solid lipid nanoparticles: a modern formulation approach in drug delivery system. Indian $J$ Pharm Sci. 2009;71:349-358. doi:10.4103/0250-474X.57282

91. Jain A, Singh SK, Kundu SC, et al. Protein nanoparticles: promising platforms for drug delivery applications. ACS Biomater Sci Eng. 2018;4:3939. doi:10.1021/acsbiomaterials.8b01098

92. Sahana DK, Mittal G, Bhardwaj V, et al. PLGA Nanoparticles for oral delivery of hydrophobic drugs: influence of organic solvent on nanoparticle formation and release behavior in vitro and in vivo using estradiol as a model drug. J Pharm Sci. 2008;97:15301542. doi: $10.1002 / j p s .21158$

93. Sahoo SK, Panyam J, Prabha S, et al. Residual polyvinyl alcohol associated with poly (d, 1-lactide-co-glycolide) nanoparticles affects their physical properties and cellular uptake. $J$ Controlled Release. 2002;82:105-114. doi:10.1016/S0168-3659 (02)00127-X

94. Pisani E, Fattal E, Paris J, et al. Surfactant dependent morphology of polymeric capsules of perfluorooctyl bromide: influence of polymer adsorption at the dichloromethane-water interface. $J$ coll interface sci. 2008;1:66-71. doi:10.1016/j.jcis.2008.07.013

95. Ramirez J, Flores-Villasenor SE, Vargas-Reyes E, et al. Preparation of PDLLA and PLGA nanoparticles stabilized with PVA and a PVA-SDS mixture: studies on particle size, degradation and drug release. J Drug Delivery Sci Tech. 2000;60:101907. doi:10.1016/j.jddst.2020.101907

96. Fornaguera C, Solans C. Methods for the in vitro characterization of nanomedicines-biological component interaction. $J$ Personalized Med. 2017;7. doi:10.3390/jpm7010002

97. Ho LWC, Liu Y, Han R, et al. Nano-cell interactions of noncationic bionanomaterials. Acc Chem Res. 2019;52:1519-1530. doi:10.1021/acs.accounts.9b00103

98. Rai R, Alwani S, Badea I. Polymeric nanoparticles in gene therapy: new avenues of design and optimization for delivery applications. Polymers. 2019;11:745. doi:10.3390/polym11040745

99. Yu B, Zhang Y, Zheng W, et al. Positive Surface Charge Enhances Selective Cellular Uptake and Anticancer Efficacy of Selenium Nanoparticles. Inorg Chem. 2012;51:8956-8963. doi:10.1021/ ic $301050 \mathrm{v}$
100. Rietwyk S, Peer D. Next-generation lipids in RNA interference therapeutics. ACS Nano. 2017;11(8):7572-7586. doi:10.1021/ acsnano. $7 \mathrm{~b} 04734$

101. Jayaraman M, Ansell SM, Mui BL, et al. Maximizing the Potency of siRNA Lipid Nanoparticles for Hepatic Gene Silencing In Vivo. Angewandte Chemie Int Edition. 2012;51(34):8529-8533. doi:10.1002/anie.201203263

102. Zhang Y, Li Y, Ma J, et al. Convenient preparation of chargeadaptive chitosan nanomedicines for extended blood circulation and accelerated endosomal escape. Nano Res. 2018;11(8):42784292. doi:10.1007/s12274-018-2014-z

103. Fay F, Quinn DJ, Gilmore BF, et al. Gene delivery using dimethyldidodecylammonium bromide-coated PLGA nanoparticles. Biomaterials. 2010;31(14):4214-4222. doi:10.1016/j. biomaterials.2010.01.143

104. Surendran SP, Moon MJ, Park R, et al. Bioactive nanoparticles for cancer immunotherapy. Int J Mol Sci. 2018;19(12):3877. doi:10.3390/ijms 19123877

105. Park W, Heo Y, Han DK. New opportunities for nanoparticles in cancer immunotherapy. Biomaterials Res. 2018;22(1):24. doi:10.1186/s40824-018-0133-y

106. Kedmi R, Ben-Arie N, Peer D. The systemic toxicity of positively charged lipid nanoparticles and the role of toll-like receptor 4 in immune activation. Biomaterials. 2010;31(26):6867-6875. doi:10.1016/j.biomaterials.2010.05.027

107. Fröhlich E. The role of surface charge in cellular uptake and cytotoxicity of medical nanoparticles. Int $J$ Nanomedicine. 2012;7:5577-5591. doi:10.2147/IJN.S36111

108. Ganji N, Bothun GD. Albumin protein coronas render nanoparticles surface active: consonant interactions at air-water and at lipid monolayer interfaces. Environ Sci. 2021;8:160-173. doi:10.1039/D0EN00934B

109. Abdelkhaliq A, Zande M, Punt A, et al. Impact of nanoparticle surface functionalization on the protein corona and cellular adhesion, uptake and transport. J Nanobiotechnology. 2018;16(1):70. doi:10.1186/s12951-018-0394-6

110. Gossmann R, Langer K, Mulac D, Antopolsky M. New perspective in the formulation and characterization of didodecyldimethylammonium bromide (DMAB) stabilized poly(Lactic-co-glycolic acid) (PLGA) nanoparticles. PLoS One. 2015;10(7):e0127532. doi:10.1371/journal.pone.0127532

111. Blanco E, Shen H, Ferrari M. Principles of nanoparticle design for overcoming biological barriers to drug delivery. Nat Biotechnol. 2015;33:941-951. doi:10.1038/nbt.3330

112. Moghimi SM. Exploiting bone marrow microvascular structure for drug delivery and future therapies. Adv Drug Deliv Rev. 1995;17(1):61-73. doi:10.1016/0169-409X(95)00041-5

113. Poznansky MJ, Juliano RL. Biological approaches to the controlled delivery of drugs: a critical review. Pharmacol Rev. 1984;36(4):277-336.

114. Tenzer S, Docter D, Kuharev J, et al. Rapid formation of plasma protein corona critically affects nanoparticle pathophysiology. Nat Nanotechnol. 2013;8(10):772-781. doi:10.1038/ nnano.2013.181

115. Sempf K, Arrey T, Gelperina S, et al. Adsorption of plasma proteins on uncoated PLGA nanoparticles. Eur J Pharm Biopharmaceutics. 2013;85(1):53-60. doi:10.1016/j.ejpb.2012. 11.030

116. Salvati A, Pitek AS, Monopoli MP, et al. Transferrin-functionalized nanoparticles lose their targeting capabilities when a biomolecule corona adsorbs on the surface. Nat Nanotechnol. 2013;8 (2):137-143. doi:10.1038/nnano.2012.237

117. Wang F, Wu Y, Zhang J, et al. Induction of cytochrome P450 involved in the accelerated blood clearance phenomenon induced by PEGylated liposomes in vivo. Drug Metabo Disposition. 2019;47(4):364-376. doi:10.1124/dmd.118.085340 
118. Jiao J, Jiao X, Wang C, et al. The contribution of PEG molecular weights in PEGylated emulsions to the various phases in the accelerated blood clearance (ABC) phenomenon in rats. AAPS PharmSciTech. 2020;21(8):300. doi:10.1208/s12249-020-01838-2

119. Kozma GT, Shimizu T, Ishida T, et al. Anti-PEG antibodies: properties, formation, testing and role in adverse immune reactions to PEGylated nano-biopharmaceuticals. Adv Drug Deliv Rev. 2020;154-155:163-175. doi:10.1016/j.addr.2020.07.024

120. Su Y, Tang W, Song Y, et al. Mixed PEGylated surfactant modifying system decrease the accelerated blood clearance phenomenon of nanoemulsions in rats. Asian J Pharm Sci. 2017;12 (1):28-36. doi:10.1016/j.ajps.2016.07.003

121. Jain D, Athawale R, Bajaj A, et al. Studies on stabilization mechanism and stealth effect of poloxamer 188 onto PLGA nanoparticles. Colloids Surf B Biointerfaces. 2013;109:59-67. doi:10.1016/j.colsurfb.2013.03.027

122. Liao H, Zhao S, Wang H, et al. $<$ p $>$ Self-Assembly Of Retinoid Nanoparticles For Melanoma Therapy. Int J Nanomedicine. 2019;14:7963-7973. doi:10.2147/IJN.S196974

123. Owens DE, Peppas NA. Opsonization, biodistribution, and pharmacokinetics of polymeric nanoparticles. Int J Pharm. 2006;307 (1):93-102. doi:10.1016/j.ijpharm.2005.10.010

124. Wani TU, Raza SN, Khan NA. Nanoparticle opsonization: forces involved and protection by long chain polymers. Polymer Bulletin. 2020;77(7):3865-3889. doi:10.1007/s00289-019-02924-7

125. Gulati NM, Stewart PL, Steinmetz NF. Bioinspired shielding strategies for nanoparticle drug delivery applications. Mol Pharm. 2018;15(8):2900-2909. doi:10.1021/acs.molpharmaceut. $8 \mathrm{~b} 00292$

126. Gref R, Lück M, Quellec P, et al. 'Stealth' corona-core nanoparticles surface modified by polyethylene glycol (PEG): influences of the corona (PEG chain length and surface density) and of the core composition on phagocytic uptake and plasma protein adsorption. Colloids Surf B Biointerfaces. 2000;18(3-4):301313. doi:10.1016/S0927-7765(99)00156-3

127. Torchilin VP, Trubetskoy VS. Which polymers can make nanoparticulate drug carriers long-circulating? Adv Drug Deliv Rev. 1995;16(2-3):141-155. doi:10.1016/0169-409X(95)00022-Y

128. Woodle MC, Lasic DD. Sterically stabilized liposomes. Biochimica et Biophysica Acta. 1992;1113(2):171-199. doi:10.1016/0304-4157(92)90038-C

129. Zhao Y, Wang Z, Zhang W, et al. Adsorbed Tween 80 is unique in its ability to improve the stability of gold nanoparticles in solutions of biomolecules. Nanoscale. 2010;2(10):2114-2119. doi:10.1039/C0NR00309C

130. Torcello-Gómez A, Santander-Ortega MJ, Peula-García JM, et al. Adsorption of antibody onto Pluronic F68-covered nanoparticles: link with surface properties. Soft Matter. 2011;7(18):8450-8461. doi:10.1039/C1SM05570D

131. Kerwin BA. Polysorbates 20 and 80 used in the formulation of protein biotherapeutics: structure and degradation pathways. $J$ Pharm Sci. 2008;97(8):2924-2935. doi:10.1002/jps.21190

132. Moghimi SM, Hunter AC, Dadswell CM, et al. Causative factors behind poloxamer 188 (Pluronic F68, Flocor ${ }^{\mathrm{TM}}$ )-induced complement activation in human sera: a protective role against poloxamer-mediated complement activation by elevated serum lipoprotein levels. Biochimica et Biophysica Acta. 2004;1689 (2):103-113. doi:10.1016/j.bbadis.2004.02.005

133. Labrenz SR. Ester Hydrolysis of Polysorbate 80 in $\mathrm{mAb}$ Drug Product: evidence in Support of the Hypothesized Risk After the Observation of Visible Particulate in mAb Formulations. J Pharm Sci. 2014;103(8):2268-2277. doi:10.1002/jps.24054

134. Gagliardi A, Paolino D, Costa N, et al. Zein- vs PLGA-based nanoparticles containing rutin: a comparative investigation. Mater Sci Eng. 2021;118:111538. doi:10.1016/j.msec.2020.111538
135. Alexis F, Pridgen E, Molnar LK, et al. Factors affecting the clearance and biodistribution of polymeric nanoparticles. $\mathrm{Mol}$ Pharm. 2008;5(4):505-515. doi:10.1021/mp800051m

136. Tang T, Jiang H, Yu Y, et al. A new method of wound treatment: targeted therapy of skin wounds with reactive oxygen speciesresponsive nanoparticles containing SDF-1 $\alpha$. Int J Nanomedicine. 2015;10:6571-6585. doi:10.2147/ijn.S88384

137. Yu SS, Lau L, Thomas T, et al. Size- and charge-dependent nonspecific uptake of PEGylated nanoparticles by macrophages. Int $J$ Nanomedicine. 2012;7:799-813. doi:10.2147/ijn.S28531

138. Parhi P, Suklabaidya S, Sahoo SK. Enhanced anti-metastatic and anti-tumorigenic efficacy of Berbamine loaded lipid nanoparticles in vivo. Sci Rep. 2017;7(1):5806. doi:10.1038/s41598-017-05296-y

139. Hall CN, Reynell C, Gesslein B, et al. Capillary pericytes regulate cerebral blood flow in health and disease. Nature. 2014;508 (7494):55-60. doi:10.1038/nature13165

140. Kutscher HL, Chao P, Deshmukh M, et al. Threshold size for optimal passive pulmonary targeting and retention of rigid microparticles in rats. $J$ Controlled Release. 2010;143(1):31-37. doi:10.1016/j.jconrel.2009.12.019

141. Moghimi SM, Hunter AC, Andresen TL. Factors controlling nanoparticle pharmacokinetics: an integrated analysis and perspective. Annu Rev Pharmacol Toxicol. 2012;52(1):481-503. doi:10.1146/annurev-pharmtox-010611-134623

142. Cataldi M, Vigliotti C, Mosca T, et al. Emerging role of the spleen in the pharmacokinetics of monoclonal antibodies, nanoparticles and exosomes. Int J Mol Sci. 2017;18(6):1249. doi:10.3390/ ijms18061249

143. Rao J. Shedding light on tumors using nanoparticles. ACS Nano. 2008;2(10):1984-1986. doi:10.1021/nn800669n

144. Matsumura Y, Maeda H. A new concept for macromolecular therapeutics in cancer chemotherapy: mechanism of tumoritropic accumulation of proteins and the antitumor agent smancs. Cancer Res. 1986;46(12 Pt 1):6387-6392.

145. Nakamura Y, Mochida A, Choyke PL, et al. Nanodrug delivery: is the enhanced permeability and retention effect sufficient for curing cancer? Bioconjug Chem. 2016;27(10):2225-2238. doi:10.1021/acs.bioconjchem.6b00437

146. Yang B, Chen Y, Shi J. Tumor-specific chemotherapy by nanomedicine-enabled differential stress sensitization. Angewandte Chemie Int Edition. 2020;59(24):9693-9701. doi:10.1002/ anie. 202002306

147. Hu F, Zhang R, Guo W, et al. PEGylated-PLGA nanoparticles coated with $\mathrm{pH}$ responsive tannic acid-Fe(III) complexes for reduced premature doxorubicin release and enhanced targeting in breast cancer. Mol Pharm. 2020. doi:10.1021/acs. molpharmaceut.0c00321

148. Liu T, Lu T, Chang S, et al. Dual stimuli-guided lipid-based delivery system of cancer combination therapy. $J$ Controlled Release. 2020;318:16-24. doi:10.1016/j.jconrel.2019.12.002

149. Tang L, Yang X, Yin Q, et al. Investigating the optimal size of anticancer nanomedicine. Proce National Acad Sci. 2014;111 (43):15344-15349. doi:10.1073/pnas.1411499111

150. Yao Y, Zhou Y, Liu L, et al. Nanoparticle-based drug delivery in cancer therapy and its role in overcoming drug resistance. Fronti Mol Biosci. 2020;7:193. doi:10.3389/fmolb.2020.00193

151. Kano $\mathrm{MR}$, Bae $\mathrm{Y}$, Iwata $\mathrm{C}$, et al. Improvement of cancer-targeting therapy, using nanocarriers for intractable solid tumors by inhibition of TGF-beta signaling. Proce National Acad Sci. 2007;104 (9):3460-3465. doi:10.1073/pnas.0611660104

152. Sindhwani S, Syed AM, Ngai J, et al. The entry of nanoparticles into solid tumours. Nat Mater. 2020;19(5):566-575. doi:10.1038/ s41563-019-0566-2

153. Tran S, DeGiovanni P, Piel B, et al. Cancer nanomedicine: a review of recent success in drug delivery. Clin Transl Med. 2017;6(1):44. doi:10.1186/s40169-017-0175-0 
154. Montaseri H, Kruger CA, Abrahamse H, et al. Review: organic nanoparticle based active targeting for photodynamic therapy treatment of breast cancer cells. Oncotarget. 2020;11(22):2120 2136. doi:10.18632/oncotarget. 27596

155. Cheng Z, Al Zaki A, Hui JZ, et al. Multifunctional nanoparticles: cost versus benefit of adding targeting and imaging capabilities. Science. 2012;338(6109):903-910. doi:10.1126/science.1226338

156. Acharya S, Dilnawaz F, Sahoo SK. Targeted epidermal growth factor receptor nanoparticle bioconjugates for breast cancer therapy. Biomaterials. 2009;30(29):5737-5750. doi:10.1016/j. biomaterials.2009.07.008

157. Poon Z, Lee JA, Huang S, et al. Highly stable, ligand-clustered "patchy" micelle nanocarriers for systemic tumor targeting. Nanomedicine. 2011;7(2):201-209. doi:10.1016/j.nano.2010.07.008

158. Alkilany AM, Zhu L, Weller H, et al. Ligand density on nanoparticles: a parameter with critical impact on nanomedicine. $A d v$ Drug Deliv Rev. 2019;143:22-36. doi:10.1016/j.addr.2019.05.010

159. Bobo D, Robinson KJ, Islam J, et al. Nanoparticle-based medicines: a review of FDA-approved materials and clinical trials to date. Pharm Res. 2016;33(10):2373-2387. doi:10.1007/s11095-016-1958-5

160. Patra JK, Das G, Fraceto LF, et al. Nano based drug delivery systems: recent developments and future prospects. $J$ Nanobiotechnology. 2018;16:71. doi:10.1186/s12951-018-0392-8

161. Chauhan VP, Stylianopoulos T, Boucher Y, et al. Delivery of molecular and nanoscale medicine to tumors: transport barriers and strategies. Annu Rev Chem Biomol Eng. 2011;2(1):281-298. doi:10.1146/annurev-chembioeng-061010-114300

162. Phi LTH, Sari IN, Yang Y, et al. Cancer stem cells (CSCs) in drug resistance and their therapeutic implications in cancer treatment. Stem Cells Int. 2018;2018:5416923. doi:10.1155/2018/5416923

163. Assaraf YG, Brozovic A, Gonçalves AC, et al. The multi-factorial nature of clinical multidrug resistance in cancer. Drug Resist Updates. 2019;46:100645. doi:10.1016/j.drup.2019.100645

164. Zuchero Y, Chen X, Bien-Ly N, et al. Discovery of novel blood-brain barrier targets to enhance brain uptake of therapeutic antibodies. Neuron. 2016;89(1):70-82. doi:10.1016/j.neuron.2015.11.024

165. Abbott NJ, Patabendige AAK, Dolman DEM, et al. Structure and function of the blood-brain barrier. Neurobiol Dis. 2010;37 (1):13-25. doi:10.1016/j.nbd.2009.07.030

166. Samal J, Rebelo AL, Pandit A. A window into the brain: tools to assess pre-clinical efficacy of biomaterials-based therapies on central nervous system disorders. Adv Drug Deliv Rev. 2019;148:68-145. doi:10.1016/j.addr.2019.01.012

167. Aggarwal P, Hall JB, McLeland CB, et al. Nanoparticle interaction with plasma proteins as it relates to particle biodistribution, biocompatibility and therapeutic efficacy. Adv Drug Deliv Rev 2009;61(6):428-437. doi:10.1016/j.addr.2009.03.009

168. Gessner A, Olbrich C, Schröder W, et al. The role of plasma proteins in brain targeting: species dependent protein adsorption patterns on brain-specific lipid drug conjugate (LDC) nanoparticles. Int J Pharm. 2001;214(1-2):87-91. doi:10.1016/S0378-5173 (00)00639-6

169. Wang J, Bruin MAC, Gan C, et al. Brain accumulation of tivozanib is restricted by $\mathrm{ABCB} 1$ (P-glycoprotein) and ABCG2 (breast cancer resistance protein) in mice. Int $J$ Pharm. 2020;581:119277. doi:10.1016/j.ijpharm.2020.119277

170. Regev R, Assaraf Yehuda G, Eytan GD, et al. Membrane fluidization by ether, other anesthetics, and certain agents abolishes P-glycoprotein ATPase activity and modulates efflux from multidrug-resistant cells. Eur J Biochem. 1999;259(1-2):18-24. doi:10.1046/j.1432-1327.1999.00037.x

171. Koffie RM, Farrar CT, Saidi L-J, et al. Nanoparticles enhance brain delivery of blood-brain barrier-impermeable probes for in vivo optical and magnetic resonance imaging. Proce National Acad Sci. 2011;108(46):18837-18842. doi:10.1073/pnas.11114 05108
172. Wilson B, Samanta MK, Santhi K, et al. Poly(n-butylcyanoacrylate) nanoparticles coated with polysorbate 80 for the targeted delivery of rivastigmine into the brain to treat Alzheimer's disease. Brain Res. 2008;1200:159-168. doi:10.1016/j.brainres.2008.01.039

173. Tahara K, Miyazaki Y, Kawashima Y, et al. Brain targeting with surface-modified poly(d, l-lactic-co-glycolic acid) nanoparticles delivered via carotid artery administration. Eur J Pharm Biopharmaceutics. 2011;77(1):84-88. doi:10.1016/j.ejpb.2010.11.002

174. Ishak RAH, Mostafa NM, Kamel AO. Stealth lipid polymer hybrid nanoparticles loaded with rutin for effective brain delivery - comparative study with the gold standard (Tween 80): optimization, characterization and biodistribution. Drug Deliv. 2017;24 (1):1874-1890. doi:10.1080/10717544.2017.1410263

175. Lei C, Davoodi P, Zhan W, et al. Development of nanoparticles for drug delivery to brain tumor: the effect of surface materials on penetration into brain tissue. J Pharm Sci. 2019;108(5):17361745. doi:10.1016/j.xphs.2018.12.002

176. Miyazawa T, Nakagawa $K$, Harigae $T$, et al. Distribution of $\beta$ carotene-encapsulated polysorbate 80 -coated poly(D, L-lactideco-glycolide) nanoparticles in rodent tissues following intravenous administration. Int $J$ Nanomedicine. 2015;10:7223-7230. doi:10.2147/ijn.S94336

177. Tröster SD, Kreuter J. Influence of the surface properties of low contact angle surfactants on the body distribution of $14 \mathrm{C}$-poly (methyl methacrylate) nanoparticles. J Microencapsul. 1992;9 (1):19-28. doi:10.3109/02652049209021219

178. Lin H, Gebhardt M, Bian S, et al. Enhancing effect of surfactants on fexofenadine $\cdot \mathrm{HCl}$ transport across the human nasal epithelial cell monolayer. Int J Pharm. 2007;330(1-2):23-31. doi:10.1016/j. ijpharm.2006.08.043

179. Jeong JH, Kang SH, Kim JH, et al. Protective effects of poly (lactic-co-glycolic acid) nanoparticles loaded with erythropoietin stabilized by sodium cholate against glutamate-induced neurotoxicity. J Nanosci Nanotechnol. 2014;14(11):8365-8371. doi:10.1166/jnn.2014.9927

180. Jeong JH, Kang SH, Kim DK, et al. Protective Effect of Cholic Acid-Coated Poly Lactic-Co-Glycolic Acid (PLGA) Nanoparticles Loaded with Erythropoietin on Experimental Stroke. J Nanosci Nanotechnol. 2019;19(10):6524-6533. doi:10.1166/jnn.2019.17078

181. Castillo-Armengol J, Fajas L, Lopez-Mejia IC. Inter-organ communication: a gatekeeper for metabolic health. EMBO Rep. 2019;20(9):e47903. doi:10.15252/embr.201947903

182. Faraday M. The Bakerian Lecture: experimental relations of gold (and other metals) to light. Philosophical Transactions Royal Soc London. 1857;147:145-181. doi:10.1098/rstl.1857.0011

183. Turkevich J, Stevenson PC, Hillier J. A study of the nucleation and growth processes in the synthesis of colloidal gold. Discuss Faraday Soc. 1951;11:55-75. doi:10.1039/DF9511100055

184. Souza CDD, Nogueira BR, Rostelato MECM. Review of the methodologies used in the synthesis gold nanoparticles by chemical reduction. J Alloys Compd. 2019;798:714-740. doi:10. 1016/j.jallcom.2019.05.153

185. Khlebtsov N, Dykman L. Biodistribution and toxicity of engineered gold nanoparticles: a review of in vitro and in vivo studies. Chem. Soc. Rev. 2011;40(3):1647-1671. doi:10.1039/C0CS00 $018 \mathrm{C}$

186. Boisselier E, Astruc D. Gold nanoparticles in nanomedicine: preparations, imaging, diagnostics, therapies and toxicity. Chem Soc Rev. 2009;38(6):1759-1782. doi:10.1039/b806051g

187. Giljohann DA, Seferos DS, Daniel WL, et al. Gold Nanoparticles for Biology and Medicine. Angewandte Chemie Int Edition. 2010;49(19):3280-3294. doi:10.1002/anie.200904359

188. Sun Y, Xia Y. Gold and silver nanoparticles: a class of chromophores with colors tunable in the range from 400 to $750 \mathrm{~nm}$ Analyst. 2003;128(6):686-691. doi:10.1039/B212437H 
189. Lee T, Mohammadniaei M, Zhang H, et al. Single Functionalized pRNA/Gold Nanoparticle for Ultrasensitive MicroRNA Detection Using Electrochemical SurfaceEnhanced Raman Spectroscopy. Adv Sci. 2020;7(3):1902477. doi:10.1002/advs.201902477

190. Zhang Y, Gu Y, He J, et al. Ultrabright gap-enhanced Raman tags for high-speed bioimaging. Nat Commun. 2019;10(1):3905. doi:10.1038/s41467-019-11829-y

191. Sharma G, Jagtap JM, Parchur AK, et al. Heritable modifiers of the tumor microenvironment influence nanoparticle uptake, distribution and response to photothermal therapy. Theranostics. 2020;10(12):5368-5383. doi:10.7150/thno.41171

192. Ma Z, Zhang Y, Zhang J, et al. Ultrasmall peptide-coated platinum nanoparticles for precise NIR-II photothermal therapy by mitochondrial targeting. ACS Appl Mater Interfaces. 2020. doi:10.1021/acsami.0c11469

193. Mantri Y, Jokerst JV. Engineering plasmonic nanoparticles for enhanced photoacoustic imaging. ACS Nano. 2020;14(8):94089422. doi:10.1021/acsnano.0c05215

194. Darweesh RS, Ayoub NM, Nazzal S. <p>Gold nanoparticles and angiogenesis: molecular mechanisms and biomedical applications. Int J Nanomedicine. 2019;14:7643-7663. doi:10.2147/IJN. S223941

195. Iravani S, Korbekandi H, Mirmohammadi SV, et al. Synthesis of silver nanoparticles: chemical, physical and biological methods. Res Pharm Sci. 2014;9(6):385-406.

196. Singh AV, Jahnke T, Kishore V, et al. Cancer cells biomineralize ionic gold into nanoparticles-microplates via secreting defense proteins with specific gold-binding peptides. Acta Biomaterialia. 2018;71:61-71. doi:10.1016/j.actbio.2018.02.022

197. Singh AV, Alapan Y, Jahnke T, et al. Seed-mediated synthesis of plasmonic gold nanoribbons using cancer cells for hyperthermia applications. J Materials Chem B. 2018;6(46):7573-7581. doi:10.1039/c8tb02239a

198. Singh AV, Jungnickel H, Leibrock L, et al. ToF-SIMS 3D imaging unveils important insights on the cellular microenvironment during biomineralization of gold nanostructures. Sci Rep. 2020;10 (1):261. doi:10.1038/s41598-019-57136-w

199. Singh AV, Batuwangala M, Mundra R, et al. Biomineralized anisotropic gold microplate-macrophage interactions reveal frustrated phagocytosis-like phenomenon: a novel paclitaxel drug delivery vehicle. ACS Appl Mater Interfaces. 2014;6(16):1467914689. doi:10.1021/am504051b

200. Singh V, Kumar V, Kashyap S, et al. Graphene Oxide Synergistically Enhances Antibiotic Efficacy in VancomycinResistant Staphylococcus aureus. ACS Applied Bio Materials. 2019;2(3):1148-1157. doi:10.1021/acsabm.8b00757

201. Libutti SK, Paciotti GF, Byrnes AA, et al. Phase I and pharmacokinetic studies of CYT-6091, a novel PEGylated colloidal goldrhTNF nanomedicine. Clin Cancer Res. 2010;16(24):6139-6149. doi:10.1158/1078-0432.CCR-10-0978

202. Gad SC, Sharp KL, Montgomery C, et al. Evaluation of the Toxicity of Intravenous Delivery of Auroshell Particles (GoldSilica Nanoshells). Int $J$ Toxicol. 2012;31(6):584-594. doi:10.1177/1091581812465969

203. Peters RJB, Oomen AG, van Bemmel G, et al. Silicon dioxide and titanium dioxide particles found in human tissues. Nanotoxicology. 2020;14(3):420-432. doi:10.1080/17435390.20 20.1718232

204. Younes M, Aggett P; EFSA Panel on Food Additives and Nutrient Sources added to Food (ANS). Re-evaluation of silicon dioxide (E 551) as a food additive. EFSA J. 2018;16:e5088. doi:10.2903/ j.efsa.2018.5088

205. Couleaud P, Morosini V, Frochot C, et al. Silica-based nanoparticles for photodynamic therapy applications. Nanoscale. 2010;2 (7):1083-1095. doi:10.1039/C0NR00096E
206. Stöber W, Fink A, Bohn E. Controlled growth of monodisperse silica spheres in the micron size range. $J$ Colloid Interface Sci. 1968;26(1):62-69. doi:10.1016/0021-9797(68)90272-5

207. Osseo-Asare K, Arriagada FJ. Preparation of $\mathrm{SiO} 2$ nanoparticles in a non-ionic reverse micellar system. Colloids Surfaces. 1990;50:321-339. doi:10.1016/0166-6622(90)80273-7

208. Iqbal MZ, Ma X, Chen T, et al. Silica-coated super-paramagnetic iron oxide nanoparticles (SPIONPs): a new type contrast agent of T 1 magnetic resonance imaging (MRI). J Materials Chem $B$. 2015;3(26):5172-5181. doi:10.1039/C5TB00300H

209. Yanagisawa T, Shimizu T, Kuroda K, et al. The Preparation of alkyltriinethylaininonium-kaneinite complexes and their conversion to microporous materials. Bull Chem Soc Jpn. 1990;63 (4):988-992. doi:10.1246/bcsj.63.988

210. Kresge CT, Leonowicz ME, Roth WJ, et al. Ordered mesoporous molecular sieves synthesized by a liquid-crystal template mechanism. Nature. 1992;359(6397):710-712. doi:10.1038/359710a0

211. Li Y, Li N, Pan W, et al. Hollow mesoporous silica nanoparticles with tunable structures for controlled drug delivery. ACS Appl Mater Interfaces. 2017;9(3):2123-2129. doi:10.1021/acsami.6b13876

212. Gisbert-Garzarán M, Vallet-Regí M. Influence of the Surface Functionalization on the Fate and Performance of Mesoporous Silica Nanoparticles. Nanomaterials. 2020;10(5):10. doi:10.3390/ nano10050916

213. Slowing II, Wu C, Vivero-Escoto JL, et al. Mesoporous silica nanoparticles for reducing hemolytic activity towards mammalian red blood cells. Small. 2009;5(1):57-62. doi:10.1002/smll.200800926

214. He Q, Shi J, Zhu M, et al. The three-stage in vitro degradation behavior of mesoporous silica in simulated body fluid. Microporous Mesoporous Mater. 2010;131(1-3):314-320. doi:10.1016/j.micromeso.2010.01.009

215. Nash T, Allison AC, Harington JS. Physico-chemical properties of silica in relation to its toxicity. Nature. 1966;210(5033):259 261. doi:10.1038/210259a0

216. Urata C, Yamada H, Wakabayashi R, et al. Aqueous colloidal mesoporous nanoparticles with ethenylene-bridged silsesquioxane frameworks. $J$ Am Chem Soc. 2011;133(21):8102-8105. doi: $10.1021 / \mathrm{ja} 201779 \mathrm{~d}$

217. Yan J, Xu X, Zhou J, et al. Fabrication of a $\mathrm{pH} /$ redox-triggered mesoporous silica-based nanoparticle with microfluidics for anticancer drugs doxorubicin and paclitaxel codelivery. ACS Applied Bio Materials. 2020;3(2):1216-1225. doi:10.1021/acsabm.9b01111

218. Chen L, Liu J, Zhang Y, et al. The toxicity of silica nanoparticles to the immune system. Nanomedicine. 2018;13(15):1939-1962. doi:10.2217/nnm-2018-0076

219. Mossman BT, Churg A. Mechanisms in the pathogenesis of Asbestosis and Silicosis. Am J Respir Crit Care Med. 1998;157 (5):1666-1680. doi:10.1164/ajrccm.157.5.9707141

220. Wong XY, Sena-Torralba A, Alvarez-Diduk R, et al. Nanomaterials for nanotheranostics: tuning their properties according to disease needs. ACS Nano. 2020;14(3):2585-2627. doi:10.1021/acsnano.9b08133

221. McCarroll IE, Bagot PAJ, Devaraj A, et al. New frontiers in atom probe tomography: a review of research enabled by cryo and/or vacuum transfer systems. Materials Today. Advances. 2020;7:10087. doi:10.1016/j.mtadv.2020.100090

222. Mosselhy DA, Assad M, Sironen T, et al. Nanotheranostics: a Possible Solution for Drug-Resistant Staphylococcus aureus and their Biofilms? Nanomaterials. 2021;11(1):82. doi:10.3390/nano11010082

223. Prasad R, Jain NK, Conde J, et al. Localized nanotheranostics: recent developments in cancer nanomedicine. Mater Today Adv. 2020;8:100087. doi:10.1016/j.mtadv.2020.100087

224. Schiffman JD, Balakrishna RG. Quantum dots as fluorescent probes: synthesis, surface chemistry, energy transfer mechanisms, and applications. Sens Actuators B Chem. 2018;258:1191-1214. doi:10.1016/j.snb.2017.11.189 
225. Resch-Genger U, Grabolle M, Cavaliere-Jaricot S, et al. Quantum dots versus organic dyes as fluorescent labels. Nat Methods. 2008;5(9):763-775. doi:10.1038/nmeth.1248

226. Han H-S, Niemeyer E, Huang Y, et al. Quantum dot/antibody conjugates for in vivo cytometric imaging in mice. Proce National Acad Sci. 2015;112(5):1350-1355. doi:10.1073/ pnas. 1421632111

227. Kim S, Lim YT, Soltesz EG, et al. Near-infrared fluorescent type II quantum dots for sentinel lymph node mapping. Nat Biotechnol. 2004;22(1):93-97. doi:10.1038/nbt920

228. Gao L, Quan LN, Arquer FPG, et al. Efficient near-infrared lightemitting diodes based on quantum dots in layered perovskite. Nat Photonics. 2020;14(4):227-233. doi:10.1038/s41566-019-0577-1

229. Han J, Won M, Kim JH, et al. Cancer stem cell-targeted bioimaging and chemotherapeutic perspective. Chem Soc Rev. 2020;49(22):7856-7878. doi:10.1039/D0CS00379D

230. Kuo CW, Chueh D-Y, Chen P. Real-time in vivo imaging of subpopulations of circulating tumor cells using antibody conjugated quantum dots. $J$ Nanobiotechnology. 2019;17(1):26. doi:10.1186/s12951-019-0453-7

231. Ye L, Yong K-T, Liu L, et al. A pilot study in non-human primates shows no adverse response to intravenous injection of quantum dots. Nat Nanotechnol. 2012;7(7):453-458. doi:10.1038/ nnano. 2012.74

232. Jan AT, Azam M, Siddiqui K, et al. Heavy metals and human health: mechanistic insight into toxicity and counter defense system of antioxidants. Int J Mol Sci. 2015;16(12):2959229630. doi:10.3390/ijms161226183

233. Yamamoto A, Honma R, Sumita M, et al. Cytotoxicity evaluation of ceramic particles of different sizes and shapes. J Biomed Mater Res A. 2004;68A(2):244-256. doi:10.1002/jbm.a.20020

234. Liu Y, Gou H, Huang X, et al. Rational synthesis of highly efficient ultra-narrow red-emitting carbon quantum dots for NIR-II two-photon bioimaging. Nanoscale. 2020;12(3):15891601. doi:10.1039/C9NR09524A

235. Song Y, Tan J, Wang G, et al. Oxygen accelerated scalable synthesis of highly fluorescent sulfur quantum dots. Chem Sci. 2020;11(3):772-777. doi:10.1039/C9SC05019A

236. Song M, Karatutlu A, Ali I, et al. Spectroscopic super-resolution fluorescence cell imaging using ultra-small Ge quantum dots. Opt Express. 2017;25(4):4240-4253. doi:10.1364/OE.25.004240

237. Shen $\mathrm{P}$, Ohta S, Inasawa S, et al. Selective labeling of the endoplasmic reticulum in live cells with silicon quantum dots. Chem Commun. 2011;47(29):8409-8411. doi:10.1039/C1CC12713F

238. Evans RJ, Lavin B, Phinikaridou A, et al. Targeted Molecular Iron Oxide Contrast Agents for Imaging Atherosclerotic Plaque. Nanotheranostics. 2020;4(4):184-194. doi:10.7150/ntno.44712

239. Wang H, Jiang Q, Shen Y, et al. The capability of detecting small vessels beyond the conventional MRI sensitivity using iron-based contrast agent enhanced susceptibility weighted imaging. NMR Biomed. 2020;33(5):e4256. doi:10.1002/nbm.4256

240. Wang Y. Current status of superparamagnetic iron oxide contrast agents for liver magnetic resonance imaging. World $J$ Gastroenterol. 2015;21(47):13400-13402. doi:10.3748/wjg.v21. i47.13400

241. Miyazawa T, Burdeos GC, Itaya M, et al. Vitamin E: regulatory redox interactions. IUBMB Life. 2019;71(4):430-441. doi:10.10 02/iub.2008

242. Reichel D, Tripathi M, Perez JM. Biological effects of nanoparticles on macrophage polarization in the tumor microenvironment. Nanotheranostics. 2019;3(1):66-88. doi:10.7150/ntno.30 052

243. Ronaldson PT, Bendayan R. HIV-1 viral envelope glycoprotein gp120 triggers an inflammatory response in cultured rat astrocytes and regulates the functional expression of P-glycoprotein. $\mathrm{Mol}$ Pharmacol. 2006;70(3):1087-1098. doi:10.1124/mol.106.025973
244. Berguetti TS, Quintaes LSP, Pereira TH, et al. TNF- $\alpha$ modulates P-glycoprotein expression and contributes to cellular proliferation via extracellular vesicles. Cells. 2019;8(5):500. doi:10.3390/ cells 8050500

245. Radomski A, Jurasz P, Alonso D, et al. Nanoparticle-induced platelet aggregation and vascular thrombosis. $\mathrm{Br} J$ Pharmacol. 2005;146(6):882-893. doi:10.1038/sj.bjp.0706386

246. Gurunathan S, Kang M-H, Qasim M, et al. Nanoparticle-mediated combination therapy: two-in-one approach for cancer. Int $\mathrm{J} \mathrm{Mol}$ Sci. 2018;19(10):3264. doi:10.3390/ijms19103264

247. Siafaka PI, Nü O, Karantas ID, et al. Current update on nanoplatforms as therapeutic and diagnostic tools: a review for the materials used as nanotheranostics and imaging modalities. Asian $J$ Pharm Sci. 2020. doi:10.1016/j.ajps.2020.03.003

248. Chen W, Ouyang J, Liu H, et al. Black phosphorus nanosheetbased drug delivery system for synergistic photodynamic/photothermal/chemotherapy of cancer. Adv Mater. 2017;29 (5):1603864. doi:10.1002/adma.201603864

249. Wu S-Y, An SSA, Hulme J. Current applications of graphene oxide in nanomedicine. Int $J$ Nanomedicine. 2015;10 Spec Iss:9-24. doi:10.2147/IJN.S88285

250. Luo M, Feng Y, Wang T, et al. Micro-/nanorobots at work in active drug delivery. Adv Funct Mater. 2018;28(25):1706100. doi:10.1002/adfm.201706100

251. Srinivas PR, Philbert M, Vu TQ, et al. Nanotechnology research: applications in nutritional sciences. The Journal of Nutrition. 2010;140(1):119-124. doi:10.3945/jn.109.115048

252. Weiss J, Takhistov $\mathrm{P}$, McClements DJ. Functional materials in food nanotechnology. $J$ Food Sci. 2006;71(9):R107-R116. doi:10.1111/j.1750-3841.2006.00195.x

253. He X, Hwang H-M. Nanotechnology in food science: functionality, applicability, and safety assessment. J Food Drug Analysis. 2016;24(4):671-681. doi:10.1016/j.jfda.2016.06.001

254. Sekhon BS. Food nanotechnology - an overview. Nanotechnol Sci Appl. 2010;3:1-15. doi:10.2147/NSA.S8677

255. Kaushik S, Djiwanti SR. Nanotechnology for enhancing crop productivity. Nanotechnology. 2017;249-262. doi:10.1007/978981-10-4573-8 11

256. Varier KM, Gudeppu M, Chinnasamy A, et al. Nanoparticles: antimicrobial applications and its prospects. Adv Nanostruct Mater Environ Remediation. 2019:321-355. doi:10.1007/978-3030-04477-0 12

257. Lee J, Kim M, Song JH, et al. Biokinetics of food additive silica nanoparticles and their interactions with food components. Colloids Surf B Biointerfaces. 2017;150:384-392. doi:10.1016/j. colsurfb.2016.11.001

258. Cunningham FJ, Goh NS, Demirer GS, et al. Nanoparticle-mediated delivery towards advancing plant genetic engineering. Trends Biotechnol. 2018;36(9):882-897. doi:10.1016/j.tibtech.2018.03.009

259. Li Y, Wang Z, Sun L, et al. Nanoparticle-based sensors for food contaminants. TrAC Trends in Analytical Chemistry. 2019;113:74-83. doi:10.1016/j.trac.2019.01.012

260. Nile SH, Baskar V, Selvaraj D, et al. Nanotechnologies in food science: applications, recent trends, and future perspectives. Nano Micro Lett. 2020;12:45. doi:10.1007/s40820-020-0383-9

261. McClements DJ. Encapsulation, protection, and delivery of bioactive proteins and peptides using nanoparticle and microparticle systems: a review. Adv Colloid Interface Sci. 2018;253:1-22. doi:10.1016/j.cis.2018.02.002

262. Simeonidis K, Martinez-Boubeta C, Zamora-Pérez P, et al. Implementing nanoparticles for competitive drinking water purification. Environ Chem Lett. 2019;17(2):705-719. doi:10.1007/ s10311-018-00821-5

263. Carbone M, Donia DT, Sabbatella G, et al. Silver nanoparticles in polymeric matrices for fresh food packaging. J King Saud Univ. 2016;28(4):273-279. doi:10.1016/j.jksus.2016.05.004 
264. Singh T, Shukla S, Kumar P, et al. Application of nanotechnology in food science: perception and overview. Front Microbiol. 2017;8:1501. doi:10.3389/fmicb.2017.01501

265. Martirosyan A, Schneider Y. Engineered nanomaterials in food: implications for food safety and consumer health. Int J Environ Research Public Health. 2014;11:5720-5750. doi:10.3390/ijerph110605720

266. Young IS. Antioxidants in health and disease. J Clin Pathol. 2001;54(3):176-186. doi:10.1136/jcp.54.3.176

267. Fajardo AM, Bisoffi M. Chapter 18 - Curcumin analogs, oxidative stress, and prostate cancer. Cancer. 2014;191. doi:10.1016/ B978-0-12-405205-5.00018-0

268. Mecocci P, Polidori MC, Troiano L, et al. Plasma antioxidants and longevity: a study on healthy centenarians. Free Radic Biol Med. 2000;28(8):1243-1248. doi:10.1016/S0891-5849(00)00246-X

269. Miyazawa T, Suzuki T, Yasuda K, et al. Accumulation of phospholipid hydroperoxides in red blood cell membranes in Alzheimer disease. Oxygen Radicals. 1992;327-330.

270. Jayakumar R, Kusiak JW, Chrest FJ, et al. Red cell perturbations by amyloid $\beta$-protein. Biochimica Et Biophysica Acta. 2003;1622 (1):20-28. doi:10.1016/S0304-4165(03)00101-6

271. Nakagawa K, Kiko T, Miyazawa T, et al. Amyloid $\beta$-induced erythrocytic damage and its attenuation by carotenoids. FEBS Lett. 2011;585(8):1249-1254. doi:10.1016/j.febslet.2011.03.060

272. Girotti AW. Lipid hydroperoxide generation, turnover, and effector action in biological systems. J Lipid Res. 1998;39(8):15291542. doi:10.1016/S0022-2275(20)32182-9

273. Nakagawa K, Kiko T, Miyazawa T, et al. Antioxidant effect of astaxanthin on phospholipid peroxidation in human erythrocytes. The British Journal of Nutrition. 2011;105(11):1563-1571. doi:10.1017/S0007114510005398

274. Nakagawa K, Kiko T, Hatade K, et al. Antioxidant effect of lutein towards phospholipid hydroperoxidation in human erythrocytes. The British Journal of Nutrition. 2009;102(9):1280-1284. doi:10.1017/S0007114509990316

275. Ellulu M, Patimah I, Khaza'ai H, Rahmat A, Abed Y. Obesity and inflammation: the linking mechanism and the complications. Arch Med Sci. 2017;4:851-863. doi:10.5114/aoms.2016.58928

276. Miyazawa T, Nakagawa K, Kim SH, et al. Curcumin and piperine supplementation of obese mice under caloric restriction modulates body fat and interleukin-1 $\beta$. Nutr Metab. 2018;15(1):12. doi:10.1186/s12986-018-0250-6

277. Burdeos GC, Nakagawa K, Kimura F, et al. Tocotrienol attenuates triglyceride accumulation in HepG2 cells and F344 rats. Lipids. 2012;47(5):471-481. doi:10.1007/s11745-012-3659-0

278. Chen Q, Espey MG, Krishna MC, et al. Pharmacologic ascorbic acid concentrations selectively kill cancer cells: action as a prodrug to deliver hydrogen peroxide to tissues. Proce National Acad Sci. 2005;102(38):13604-13609. doi:10.1073/pnas.0506390102

279. Yun J, Mullarky E, Lu C, et al. Vitamin C selectively kills KRAS and BRAF mutant colorectal cancer cells by targeting GAPDH. Science. 2015;350(6266):1391-1396. doi:10.1126/science.aaa5004

280. Miyazawa T, Matsumoto A, Miyahara Y, et al. Determination of cellular vitamin C dynamics by HPLC-DAD. Analyst. 2019;144 (11):3483-3487. doi:10.1039/C8AN02240B

281. Pawlowska E, Szczepanska J, Blasiak J. Pro- and antioxidant effects of vitamin $\mathrm{C}$ in cancer in correspondence to Its dietary and pharmacological concentrations. Oxid Med Cell Longev. 2019;2019:7286737. doi:10.1155/2019/7286737

282. Martínez-Ballesta M, Gil-Izquierdo Á, García-Viguera C, et al. Nanoparticles and controlled delivery for bioactive compounds: outlining challenges for new "Smart-Foods" for health. Foods. 2018;7(5):72. doi:10.3390/foods 7050072

283. Donsì F, Ferrari G. Changing the vision in smart food design utilizing the next generation of nanometric delivery systems for bioactive compounds. Foods. 2020;9(8):1100. doi:10.3390/ foods 9081100
284. Diab R, Jaafar-Maalej C, Fessi H, et al. Engineered nanoparticulate drug delivery systems: the next frontier for oral administration? AAPS J. 2012;14(4):688-702. doi:10.1208/s12248-0129377-y

285. Brotons-Canto A, Gonzalez-Navarro CJ, Gurrea J, et al. Zein nanoparticles improve the oral bioavailability of resveratrol in humans. J Drug Deliv Sci Technol. 2020;57:101704. doi:10.10 16/j.jddst.2020.101704

286. Bahrami A, Delshadi R, Jafari SM, et al. Nanoencapsulated nisin: an engineered natural antimicrobial system for the food industry. Trends Food Sci Tech. 2019;94:20-31. doi:10.1016/j.tifs.2019. 10.002

287. Davis DR, Epp MD, Riordan HD. Changes in USDA food composition data for 43 garden crops, 1950 to 1999. J Am Coll Nutr. 2004;23(6):669-682. doi:10.1080/07315724.2004.10719409

288. Rieux A, Fievez V, Garinot M, et al. Nanoparticles as potential oral delivery systems of proteins and vaccines: a mechanistic approach. J Controlled Release. 2006;116:1-27. doi:10.1016/j. jconrel.2006.08.013

289. Yada RY, Buck N, Canady R, et al. Engineered nanoscale food ingredients: evaluation of current knowledge on material characteristics relevant to uptake from the gastrointestinal tract. Comprehen Rev Food Sci Food Safety. 2014;13:730-744. doi:10.1111/1541-4337.12076

290. Hosnedlova B, Kepinska M, Skalickova S, et al. Nano-selenium and its nanomedicine applications: a critical review. Int $J$ Nanomedicine. 2018;13:2107-2128. doi:10.2147/ijn.S157541

291. McClements DJ, Xiao H. Is nano safe in foods? Establishing the factors impacting the gastrointestinal fate and toxicity of organic and inorganic food-grade nanoparticles. Sci Food. 2017;1:6. doi:10.1038/s41538-017-0005-1

292. Luo Y. Perspectives on important considerations in designing nanoparticles for oral delivery applications in food. $J \mathrm{Agr}$ Food Res. 2020;2:100031. doi:10.1016/j.jafr.2020.100031

293. McClements DJ. Nanotechnology approaches for improving the healthiness and sustainability of the modern food supply. ACS Omega. 2020;5:29623-29630. doi:10.1021/acsomega.0c04050

294. Cepo DV, Radic K. Food (matrix) effects on bioaccessibility and intestinal permeability of major olive antioxidants. Foods. 2020;9:1831. doi:10.3390/foods9121831

295. Wang T, Luo Y. Biological fate of ingested lipid-based nanoparticles: current understanding and future directions. Nanoscale. 2019;11:11048-11063. doi:10.1039/C9NR03025E

296. Mackie A, Mulet-Cabero A, Torcello-Gomez A. Simulating human digestion: developing our knowledge to create healthier and more sustainable foods. Food Funct. 2020;11:9397-9431. doi:10.1039/D0FO01981J

297. Inchaurraga L, Martínez-López AL, Abdulkarim M, et al. Modulation of the fate of zein nanoparticles by their coating with a Gantrez ${ }^{\circledR}$ AN-thiamine polymer conjugate. Int J Pharm. 2019;1:100006. doi:10.1016/j.ijpx.2019.100006

298. Taipaleenmäki E, Städler B. Recent advancements in using polymers for intestinal mucoadhesion and mucopenetration. Macromol Biosci. 2020;20:1900342. doi:10.1002/mabi.201900342

299. Pridgen EM, Alexis F, Farokhzad OC. Polymeric nanoparticle technologies for oral drug delivery. Clin Gastroenterol Hepatol. 2014;12:1605-1610. doi:10.1016/j.cgh.2014.06.018

300. Maisel K, Ensign L, Reddy M, et al. Effect of surface chemistry on nanoparticle interaction with gastrointestinal mucus and distribution in the gastrointestinal tract following oral and rectal administration in the mouse. $J$ Controlled Release. 2015; 197:48-57. doi:10.1016/j.jconrel.2014.10.026

301. Bourganis V, Karamanidou T, Samaridou E, et al. On the synthesis of mucus permeating nanocarriers. Eur $J$ Pharm Biopharmaceutics. 2015;97:239-249. doi:10.1016/j.ejpb.2015. 01.021 
302. Elgadir MA, Uddin MS, Ferdosh S, et al. Impact of chitosan composites and chitosan nanoparticle composites on various drug delivery systems: a review. J Food Drug Analysis. 2015;23:619-629. doi:10.1016/j.jfda.2014.10.008

303. Liang J, Yan H, Puligundla P, et al. Applications of chitosan nanoparticles to enhance absorption and bioavailability of tea polyphenols: a review. Food Hydrocoll. 2017;69:286-292. doi:10.1016/j.foodhyd.2017.01.041

304. Newton JL, Jordan N, Pearson J, et al. The adherent gastric antral and duodenal mucus gel layer thins with advancing age in subjects infected with Helicobacter pylori. Gerontology. 2000;46: 153-157. doi:10.1159/000022151

305. Nugent SG, Kumar D, Ramption DS, et al. Intestinal luminal pH in inflammatory bowel disease: possible determinants and implications for therapy with aminosalicylates and other drugs. Gut. 2001;48:571-577. doi:10.1136/gut.48.4.571

306. Sigurdsson HH, Kirch J, Lehr C-M. Mucus as a barrier to lipophilic drugs. Int $J$ Pharm. 2013;453:56-64. doi:10.1016/j. ijpharm.2013.05.040

307. Witten J, Ribbeck K. The particle in the spider's web: transport through biological hydrogels. Nanoscale. 2017;9:8080-8095. doi:10.1039/C6NR09736G

308. Ensign LM, Lai SK, Wang -Y-Y, et al. Pretreatment of human cervicovaginal mucus with Pluronic F127 enhances nanoparticle penetration without compromising mucus barrier properties to herpes simplex virus. Biomacromolecules. 2014;15:4403-4409. doi:10.1021/bm501419z

309. Zhang X, Dong W, Cheng $\mathrm{H}$, et al. Modulating intestinal mucus barrier for nanoparticles penetration by surfactants. Asian J Pharm Sci. 2019;14:543-551. doi:10.1016/j.ajps.20 18.09.002

310. Yang M, Lai SK, Wang -Y-Y, et al. Biodegradable nanoparticles composed entirely of safe materials that rapidly penetrate human mucus. Angewandte Chemie Int Edition. 2011;50:2597-2600. doi:10.1002/anie. 201006849

311. Hillaireau H, Couvreur P. Nanocarriers' entry into the cell: relevance to drug delivery. Cell Mol Life Sci. 2009;66:2873-2896. doi:10.1007/s00018-009-0053-z

312. Jani P, Halbert GW, Langridge J, et al. Nanoparticle uptake by the rat gastrointestinal mucosa: quantitation and particle size dependency. J Pharm Pharmacol. 1990;42:821-826. doi:10.1111/ j.2042-7158.1990.tb07033.x

313. Fröhlich E, Roblegg E. Models for oral uptake of nanoparticles in consumer products. Toxicology. 2012;291:10-17. doi:10.1016/j. tox.2011.11.004

314. Rejman J, Oberle V, Zuhorn IS, et al. Size-dependent internalization of particles via the pathways of clathrin- and caveolaemediated endocytosis. Biochem J. 2004;377:159-169. doi:10. 1042/bj20031253

315. Ensign LM, Cone R, Hanes J. Oral drug delivery with polymeric nanoparticles: the gastrointestinal mucus barriers. Adv Drug Deliv Rev. 2012;64:557-570. doi:10.1016/j.addr.2011.12.009

316. Nellans HN. (B) Mechanisms of peptide and protein absorption: (1) Paracellular intestinal transport: modulation of absorption. Adv Drug Deliv Rev. 1991;7:339-364. doi:10.1016/0169-409X (91)90013-3

317. Hillyer JF, Albrecht RM. Gastrointestinal persorption and tissue distribution of differently sized colloidal gold nanoparticles. $J$ Pharm Sci. 2001;90:1927-1936. doi:10.1002/jps.1143

318. Lamson NG, Berger A, Fein KC, et al. Anionic nanoparticles enable the oral delivery of proteins by enhancing intestinal permeability. Nat Biomed Eng. 2020;4:84-96. doi:10.1038/s41551019-0465-5

319. Schoellhammer CM, Schroeder A, Maa R, et al. Ultrasoundmediated gastrointestinal drug delivery. Sci Transl Med. 2015;7:310ra168. doi:10.1126/scitranslmed.aaa5937
320. Harigae T, Nakagawa K, Miyazawa T, et al. Metabolic fate of poly-(lactic-co-glycolic acid)-based curcumin nanoparticles following oral administration. Int J Nanomedicine. 2016;11:30093022. doi: $10.2147 /$ IJN.S107442

321. Parada J, Aguilera JM. Food microstructure affects the bioavailability of several nutrients. J Food Sci. 2007;72:R21-R32. doi:10.1111/j.1750-3841.2007.00274.x

322. Itaya M, Miyazawa T, Zingg JM, et al. The differential cellular uptake of curcuminoids in vitro depends dominantly on albumin interaction. Phytomedicine. 2019;59:152902. doi:10.1016/j. phymed.2019.152902

323. Yan Y, Gause KT, Kamphuis MMJ, et al. Differential roles of the protein corona in the cellular uptake of nanoporous polymer particles by monocyte and macrophage cell lines. ACS Nano. 2013;7:10960-10970. doi:10.1021/nn404481f

324. Ashurst PR. Carbonated beverages. Reference Module Food Sci. 2016;1-5. doi:10.1016/B978-0-08-100596-5.03240-6

325. Caleb OJ, Mahajan PV, Al-Said FA, et al. Modified atmosphere packaging technology of fresh and fresh-cut produce and the microbial consequences-a review. Food Bioprocess Tech. 2013;6:303-329. doi:10.1007/s11947-012-0932-4

326. Sharma C, Dhiman R, Rokana N, et al. Nanotechnology: an untapped resource for food packaging. Front Microbiol. 2017;8:1735. doi:10.3389/fmicb.2017.01735

327. Poverenov E, Danino S, Horev B, et al. Layer-by-layer electrostatic deposition of edible coating on fresh cut melon model: anticipated and unexpected effects of alginate-chitosan combination. Food Bioprocess Tech. 2014;7:1424-1432. doi:10.1007/ s11947-013-1134-4

328. Azeredo HMC, Mattoso LHC, Avena-Bustillos RJ, et al. Nanocellulose Reinforced Chitosan Composite Films as Affected by Nanofiller Loading and Plasticizer Content. $J$ Food Sci. 2010;75:N1-N7. doi:10.1111/j.1750-3841.2009. 01386. $\mathrm{x}$

329. Shamsuri AA. A Short review on the effect of surfactants on the mechanico-thermal properties of polymer nanocomposites. Applied Sci. 2020;10:4867. doi:10.3390/app10144867

330. Dhall RK. Advances in edible coatings for fresh fruits and vegetables: a review. Crit Rev Food Sci Nutr. 2013;53:435-450. doi:10.1080/10408398.2010.541568

331. Shit SC, Shah PM. Edible polymers: challenges and opportunities. $J$ Polymers. 2014;2014:427259. doi:10.1155/2014/ 427259

332. Higashisaka K, Yoshioka Y, Tsutsumi Y. Applications and safety of nanomaterials used in the food industry. Food Safety. 2015;3:39-47. doi:10.14252/foodsafetyfscj.2015005

333. Lindequist U, Niedermeyer THJ, Jülich W. The Pharmacological Potential of Mushrooms. Evid Based Complement Alternative Med. 2005;2:906016. doi:10.1093/ecam/neh107

334. Pandy AT, Pandy I, Hachenberger Y, et al. Emerging paradigm against global antimicrobial resistance via bioprospecting of mushroom into novel nanotherapeutics development. Trends Food Sci Tech. 2020;106:333-344. doi:10.1016/j.tifs.20 20.10 .025

335. Dwivedi C, Pandey I, Misra V, et al. The prospective role of nanobiotechnology in food and food packaging products. Integrative Food Nutrition Metab. 2018:5. doi:10.15761/ IFNM.1000237

336. Kumar V, Guleria P, Metha SK, et al. Nanosensors for food quality and safety assessment. Environ Chem Lett. 2017;15:165177. doi:10.1007/s10311-017-0616-4

337. Karatapanis AE, Fiamegos Y, Stalikas D. Silica-modified magnetic nanoparticles functionalized with cetylpyridinium bromide for the preconcentration of metals after complexation with 8hydroxyquinoline. Talanta. 2011;84:834-839. doi:10.1016/j. talanta.2011.02.013 
338. Zahid A, Lashin A, Rana UA, et al. Development of surfactant based electrochemical sensor for the trace level detection of mercury. Electrochim Acta. 2016;190:1007-1014. doi:10.1016/j. electacta.2015.12.164

339. Mustafa F. Andreescu. Nanotechnology-based approaches for food sensing and packaging applications. RSC Adv. 2020;10:19309-19336. doi:10.1039/D0RA01084G

340. Wang Y, Duncan TV. Nanoscale sensors for assuring the safety of food products. Curr Opin Biotechnol. 2017;44:74-86. doi:10.1016/j.copbio.2016.10.005

341. Paladini F, Pollini M. Antimicrobial silver nanoparticles for wound healing application: progress and future trends. Materials. 2019;12:2540. doi:10.3390/ma12162540

342. Sanguansri P, Augustin MA. Nanoscale materials development a food industry perspective. Trends Food Sci Tech. 2006;17:547556. doi:10.1016/j.tifs.2006.04.010

343. Sondi I, Salopek-Sondi B. Silver nanoparticles as antimicrobial agent: a case study on E. coli as a model for Gram-negative bacteria. $J$ Colloid Interface Sci. 2004;275:177-182. doi:10.1016/j.jcis.2004.02.012

344. Kim JS, Kuk E, Yu KN, et al. Antimicrobial effects of silver nanoparticles. Nanomedicine. 2007;3:95-101. doi:10.1016/j. nano.2006.12.001

345. Franci G, Falanga A, Galdiero S, et al. Silver nanoparticles as potential antibacterial agents. Molecules. 2015;20:8856-8874. doi:10.3390/molecules20058856

346. Costa C, Conte A, Buonocore GG, et al. Calcium-alginate coating loaded with silver-montmorillonite nanoparticles to prolong the shelf-life of fresh-cut carrots. Food Res Int. 2012;48:164-169. doi:10.1016/j.foodres.2012.03.001

347. Hedayati S, Niakousari M. Effect of coatings of silver nanoparticles and gum Arabic on physicochemical and microbial properties of green bell pepper (Capsicum annuum). J Food Proce Preservation. 2015;39::2001-2007. doi:10.1111/jfpp.12440

348. Kvítek L, Panáček A, Soukupová J, et al. Effect of surfactants and polymers on stability and antibacterial activity of silver nanoparticles (NPs). J Phys Chem C. 2008;112:5825-5834. doi:10.1021/jp711616v

349. Bae S-H, Yu J, Lee TG, et al. Protein food matrix-ZnO nanoparticle interactions affect protein conformation, but may not be biological responses. Int J Mol Sci. 2018;19:3926. doi:10.3390/ ijms 19123926

350. Li X, Li W, Jiang Y, et al. Effect of nano-ZnO-coated active packaging on quality of fresh-cut 'Fuji' apple. Int J Food Sci Tech. 2011;46:1947-1955. doi:10.1111/j.1365-2621.2011.02706.x

351. Huy TQ, Thanh NTH, Thuy NT, et al. Cytotoxicity and antiviral activity of electrochemical - synthesized silver nanoparticles against poliovirus. $J$ Virol Methods. 2017;241:52-57. doi:10.1016/j.jviromet.2016.12.015

352. Sreekanth TVM, Nagajyothi PC, Muthuraman P, et al. Ultra-sonication-assisted silver nanoparticles using Panax ginseng root extract and their anti-cancer and antiviral activities. J Photochem Photobiol B. 2018;188:6-11. doi:10.1016/j.jphotobiol.2018.08.013

353. Antoine TE, Hadigal SR, Yakoub AM, et al. Intravaginal zinc oxide tetrapod nanoparticles as novel immunoprotective agents against genital herpes. $J$ Immunol. 2016;196:4566-4575. doi:10.4049/jimmunol.1502373

354. Gurunathan S, Qasim M, Choi Y, et al. Antiviral potential of nanoparticles-Can nanoparticles fight against coronaviruses? Nanomaterials. 2020;10. doi:10.3390/nano10091645

355. Voss L, Hsiao IL, Ebisch M, et al. The presence of iron oxide nanoparticles in the food pigment E172. Food Chem. 2020;327:127000. doi:10.1016/j.foodchem.2020.127000

356. Musial J, Krakowiak R, Mlynarczyk DT, et al. Titanium dioxide nanoparticles in food and personal care products-what do we know about their safety? Nanomaterials. 2020;10:1110. doi:10.3390/nano10061110
357. Go M, Bae S, Kim H, et al. Interactions between food additive silica nanoparticles and food matrices. Front Microbiol. 2017;8:1013. doi:10.3389/fmicb.2017.01013

358. Panáček A, Kvítek L, Smékalová M, et al. Bacterial resistance to silver nanoparticles and how to overcome it. Nat Nanotechnol. 2018;13:65-71. doi:10.1038/s41565-017-0013-y

359. Lamas B, Martins Breyner N, et al. Impacts of foodborne inorganic nanoparticles on the gut microbiota-immune axis: potential consequences for host health. Part Fibre Toxicol. 2020;17:19. doi:10.1186/s12989-020-00349-z

360. Liebezeit G, Liebezeit E. Synthetic particles as contaminants in German beers. Food Additives Contaminants. 2014;31:15741578. doi:10.1080/19440049.2014.945099

361. Peters RJB, Bemmel G, Herrera-Rivera Z, et al. Characterization of titanium dioxide nanoparticles in food products: analytical methods to define nanoparticles. $J$ Agric Food Chem. 2014;62:6285-6293. doi:10.1021/jf5011885

362. Peters RJB, Rivera ZH, Bemmel G, et al. Development and validation of single particle ICP-MS for sizing and quantitative determination of nano-silver in chicken meat. Anal Bioanal Chem. 2014;406:3875-3885.

363. Reed RB, Faust JJ, Yang Y, et al. Characterization of nanomaterials in metal colloid-containing dietary supplement drinks and assessment of their potential interactions after ingestion. ACS Sustain Chem Eng. 2014;2:1616-1624. doi:10.1021/sc500108m

364. Donovan AR, Adams CD, Ma Y, et al. Single particle ICP-MS characterization of titanium dioxide, silver, and gold nanoparticles during drinking water treatment. Chemosphere. 2016;144:148153.

365. Liebezeit G, Liebezeit E. Non-pollen particulates in honey and sugar. Food Additives Contaminants. 2013;30:2136-2140. doi:10.1080/19440049.2013.843025

366. Zhang L, Chen F, An H, et al. Physicochemical properties, firmness, and nanostructures of sodium carbonate-soluble pectin of 2 Chinese cherry cultivars at 2 ripening stages. J Food Sci. 2008;73:N17-N22. doi:10.1111/j.1750-3841.2008.00799.x

367. Dang JMC, Braet F, Copeland L. Nanostructural analysis of starch components by atomic force microscopy. J Microsc. 2006;224:181-186. doi:10.1111/j.1365-2818.2006.01681.x

368. Kalgaonkar S, Lönnerdal B. Receptor-mediated uptake of ferritinbound iron by human intestinal Caco-2 cells. J Nutr Biochem. 2009;20:304-311. doi:10.1016/j.jnutbio.2008.04.003

369. Powell JJ, Faria N, Thomas-McKay E, et al. Origin and fate of dietary nanoparticles and microparticles in the gastrointestinal tract. $J$ Autoimmun. 2010;34:J226-J233. doi:10.1016/j.jaut.2009.11.006

370. Jain A, Ranjan S, Dasgupta N, et al. Nanomaterials in food and agriculture: an overview on their safety concerns and regulatory issues. Crit Rev Food Sci Nutr. 2018;58:297-317. doi:10.1080/ 10408398.2016.1160363

371. Buzea C, Pacheco II, Robbie K. Nanomaterials and nanoparticles: sources and toxicity. Biointerphases. 2007;2:MR17-MR71. doi: $10.1116 / 1.2815690$

372. Lin S, Reppert J, Hu Q, et al. Uptake, translocation, and transmission of carbon nanomaterials in rice plants. Small. 2009;5:11281132. doi:10.1002/smll.200801556

373. Dan Y, Zhang W, Xue R, et al. Characterization of gold nanoparticle uptake by tomato plants using enzymatic extraction followed by single-particle inductively coupled plasma-mass spectrometry analysis. Environ Sci Technol. 2015;49:3007-3014. doi: $10.1021 / \mathrm{es} 506179 \mathrm{e}$

374. Yan A, Chen Z. Impacts of silver nanoparticles on plants: a focus on the phytotoxicity and underlying mechanism. Int $\mathrm{J} \mathrm{Mol} \mathrm{Sci}$. 2019;20:1003. doi:10.3390/ijms20051003

375. Luyckx M, Hausman J, Lutts S, et al. Silicon and Plants: current Knowledge and Technological Perspectives. Front Plant Sci. 2017;8:411. doi:10.3389/fpls.2017.00411 
376. Smita S, Gupta SK, Bartonova A, et al. Nanoparticles in the environment: assessment using the causal diagram approach Environ Health. 2012;11:S13. doi:10.1186/1476-069X-11-S1-S13

377. Bieberstein A, Roosen J, Marette S, et al. Consumer choices for nano-food and nano-packaging in France and Germany. Eur Rev Agr Econ. 2012;40:73-94. doi:10.1093/erae/jbr069

378. Singh AV, Ansari MHD, Rosenkranz D, et al. Artificial intelligence and machine learning in computational nanotoxicology: unlocking and empowering nanomedicine. Adv Healthcare Mater. 2020;9:1901862. doi:10.1002/adhm.201901862

379. Singh AV, Rosenkranz D, Ansari MHD, et al. Artificial intelligence and machine learning empower advanced biomedical material design to toxicity prediction. Adv Intelligent Sys. 2020;2:2000084. doi:10.1002/aisy.202000084

380. Singh AV, Jahnke T, Wang S, et al. Anisotropic gold nanostructures: optimization via in silico modeling for hyperthermia. ACS Applied Nano Materials. 2018;1:6205-6216. doi:10.1021/ acsanm.8b01406

381. Singh AV, Maharjan R-S, Kanase A, et al. Machine-learningbased approach to decode the influence of nanomaterial properties on their interaction with cells. ACS Applied Mater Interfaces. 2021;13:1943-1955. doi:10.1021/acsami.0c18470

382. Göppert TM, Müller RH. Polysorbate-stabilized solid lipid nanoparticles as colloidal carriers for intravenous targeting of drugs to the brain: comparison of plasma protein adsorption patterns. $J$ Drug Target. 2005;13:179-187. doi:10.1080/10611860500071292

383. Shubhra QTH, Tóth J, Gyenis J, et al. Surface modification of HSA containing magnetic PLGA nanoparticles by poloxamer to decrease plasma protein adsorption. Colloids Surf $B$ Biointerfaces. 2014;122:529-536. doi:10.1016/j.colsurfb.2014.07.025

384. Jackson JK, Springate CMK, Hunter WL, et al. Neutrophil activation by plasma opsonized polymeric microspheres: inhibitory effect of Pluronic F127. Biomaterials. 2000;21:1483-1491. doi:10.1016/S0142-9612(00)00034-X

385. Tröster SD, Müller U, Kreuter J. Modification of the body distribution of poly(methyl methacrylate) nanoparticles in rats by coating with surfactants. Int $J$ Pharm. 1990;61:85-100. doi:10.1016/0378-5173(90)90047-8

386. Mahmoud NN, Hikmat S, Ghith DA, et al. Gold nanoparticles loaded into polymeric hydrogel for wound healing in rats: effect of nanoparticles' shape and surface modification. Int J Pharm. 2019;565:174-186. doi:10.1016/j.ijpharm.2019.04.079

387. Botequim D, Maia J, Lino MMF, et al. Nanoparticles and Surfaces Presenting Antifungal, Antibacterial and Antiviral Properties Langmuir. 2012;28:7646-7656. doi:10.1021/la300948n

388. Yuan Z, Hu Y, Gao J. Brain Localization and Neurotoxicity Evaluation of Polysorbate 80-Modified Chitosan Nanoparticles in Rats. PLoS One. 2015;10:e0134722. doi:10.1371/journal. pone. 0134722

389. Cheung S, O'Shea DF. Directed self-assembly of fluorescence responsive nanoparticles and their use for real-time surface and cellular imaging. Nat Commun. 2017;8:1885. doi:10.1038/ s41467-017-02060-8

390. Wang X, Yan J, Pan D, et al. Polyphenol-Poloxamer SelfAssembled Supramolecular Nanoparticles for Tumor NIRF/PET Imaging. Adv Healthcare Mater. 2018;7:1701505. doi:10.1002/ adhm.201701505

391. Vu-Quang H, Vinding MS, Nielsen T, et al. Pluronic F127-Folate Coated Super Paramagenic Iron Oxide Nanoparticles as Contrast Agent for Cancer Diagnosis in Magnetic Resonance Imaging. Polymers. 2019;11:743. doi:10.3390/polym11040743

392. Mukherjee M, Gangopadhyay K, Das R, Purkayastha P. Development of Non-ionic Surfactant and Protein-Coated Ultrasmall Silver Nanoparticles: increased Viscoelasticity Enables Potency in Biological Applications. ACS Omega. 2020;5:8999-9006. doi:10.1021/acsomega.0c00825
393. Güney G, Kutlu HM, Genç L. Preparation and characterization of ascorbic acid loaded solid lipid nanoparticles and investigation of their apoptotic effects. Colloids Surf B Biointerfaces. 2014;121:270-280. doi:10.1016/j.colsurfb.2014.05.008

394. Escalona-Rayo O, Fuentes-Vázquez P, Jardon-Xicotencatl S, et al. Rapamycin-loaded polysorbate 80-coated PLGA nanoparticles: optimization of formulation variables and in vitro anti-glioma assessment. J Drug Deliv Sci Technol. 2019;52:488-499. doi:10.1016/j.jddst.2019.05.026

395. Suktham K, Koobkokkruad T, Wutikhun T, et al. Efficiency of resveratrol-loaded sericin nanoparticles: promising bionanocarriers for drug delivery. Int $J$ Pharm. 2018;537:48-56. doi:10.1016/j.ijpharm.2017.12.015

396. Yan F, Zhang C, Zheng Y, et al. The effect of poloxamer 188 on nanoparticle morphology, size, cancer cell uptake, and cytotoxicity. Nanomedicine. 2010;6:170-178. doi:10.1016/j.nano.2009. 05.004

397. Gupta PN, Jain S, Nehate C, et al. Development and evaluation of paclitaxel loaded PLGA: poloxamerblend nanoparticles for cancer chemotherapy. Int J Biol Macromol. 2014;69:393-399. doi:10.1016/j.ijbiomac.2014.05.067

398. Esim O, Bakirhan NK, Sarper M, et al. Influence of emulsifiers on the formation and in vitro anticancer activity of epirubicin loaded PLGA nanoparticles. J Drug Deliv Sci Technol. 2020;60:102027. doi:10.1016/j.jddst.2020.102027

399. Loureiro A, Noro J, Abreu AS, et al. Absence of Albumin Improves in Vitro Cellular Uptake and Disruption of Poloxamer 407-Based Nanoparticles inside Cancer Cells. Mol Pharm. 2018;15:527-535. doi:10.1021/acs.molpharmaceut.7b00893

400. Moura S, Noro J, Cerqueira P, et al. Poloxamer 407 based-nanoparticles for controlled release of methotrexate. Int J Pharm. 2020;575:118924. doi:10.1016/j.ijpharm.2019.118924

401. Ambruosi A, Gelperina S, Khalansky A, et al. Influence of surfactants, polymer and doxorubicin loading on the anti-tumour effect of poly (butyl cyanoacrylate) nanoparticles in a rat glioma model. $J$ Microencapsul. 2006;23:582-592. doi:10.1080/02652040600788080

402. Perepelyuk M, Thangavel C, Liu Y, et al. Biodistribution and Pharmacokinetics Study of siRNA-loaded Anti-NTSR1-mAbfunctionalized Novel Hybrid Nanoparticles in a Metastatic Orthotopic Murine Lung Cancer Model. Mol Therapy. 2016;5: e282. doi: $10.1038 / \mathrm{mtna} .2015 .56$

403. Petri B, Bootz A, Khalansky A, et al. Chemotherapy of brain tumour using doxorubicin bound to surfactant-coated poly(butyl cyanoacrylate) nanoparticles: revisiting the role of surfactants. $J$ Controlled Release. 2007;117:51-58. doi:10.1016/j.jconrel.2006.10.015

404. Ramge P, Unger RE, Oltrogge JB, et al. Polysorbate- 80 coating enhances uptake of polybutylcyanoacrylate (PBCA)-nanoparticles by human and bovine primary brain capillary endothelial cells. Eur J Neurosci. 2000;12:1931-1940. doi:10.1046/j.14609568.2000.00078.x

405. Borchard G, Audus KL, Shi F, et al. Uptake of surfactant-coated poly(methyl methacrylate)-nanoparticles by bovine brain microvessel endothelial cell monolayers. Int J Pharm. 1994;110:29-35. doi:10.1016/0378-5173(94)90372-7

406. Gulyaev AE, Gelperina SE, Skidan IN, et al. Significant Transport of Doxorubicin into the Brain with Polysorbate 80-Coated Nanoparticles. Pharm Res. 1999;16:1564-1569. doi:10.1023/ A:1018983904537

407. Wohlfart S, Khalansky AS, Gelperina S, et al. Kinetics of transport of doxorubicin bound to nanoparticles across the blood-brain barrier. J Controlled Release. 2011;154:103-107. doi:10.1016/j. jconrel.2011.05.010

408. Gao K, Jiang X. Influence of particle size on transport of methotrexate across blood brain barrier by polysorbate 80 -coated polybutylcyanoacrylate nanoparticles. Int J Pharm. 2006;310:213219. doi:10.1016/j.ijpharm.2005.11.040 
409. Wilson B, Samanta MK, Santhi K, et al. Targeted delivery of tacrine into the brain with polysorbate 80-coated poly(n-butylcyanoacrylate) nanoparticles. Eur J Pharm Biopharmaceutics. 2008;70:75-84. doi:10.1016/j.ejpb.2008.03.009

410. Kreuter J, Alyautdin RN, Kharkevich DA, et al. Passage of peptides through the blood-brain barrier with colloidal polymer particles (nanoparticles). Brain Res. 1995;674:171-174. doi:10.1016/0006-8993(95)00023-J

411. Kurakhmaeva KB, Voronina TA, Kapica IG, et al. Antiparkinsonian effect of nerve growth factor adsorbed on polybutylcyanoacrylate nanoparticles coated with polysorbate-80. Bull Exp Biol Med. 2008;145:259-262. doi:10.1007/s10517-008-0065-y

412. Khalin I, Alyautdin R, Wong TW, et al. Brain-derived neurotrophic factor delivered to the brain using poly (lactide-co-glycolide) nanoparticles improves neurological and cognitive outcome in mice with traumatic brain injury. Drug Deliv. 2016;23:35203528. doi:10.1080/10717544.2016.1199609

413. Huang Y, Zhang B, Xie S, et al. Superparamagnetic Iron Oxide Nanoparticles Modified with Tween 80 Pass through the Intact Blood-Brain Barrier in Rats under Magnetic Field. ACS Appl Mater Interfaces. 2016;8:11336-11341. doi:10.1021/acsami.6b02838

414. Dara T, Vatanara A, Sharifzadeh M, et al. Improvement of memory deficits in the rat model of Alzheimer's disease by erythropoietin-loaded solid lipid nanoparticles. Neurobiol Learn Mem. 2019;166:107082. doi:10.1016/j.nlm.2019.107082

415. Sivaji K, Kannan RR. Polysorbate 80 Coated Gold Nanoparticle as a Drug Carrier for Brain Targeting in Zebrafish Model. $J$ Cluster Sci. 2019;30:897-906. doi:10.1007/s10876-019-01548-1

416. Gelperina S, Maksimenko O, Khalansky A, et al. Drug delivery to the brain using surfactant-coated poly(lactide-co-glycolide) nanoparticles: influence of the formulation parameters. Eur J Pharm Biopharmaceutics. 2010;74:157-163. doi:10.1016/j.ejpb.2009. 09.003

417. Kreuter J, Petrov VE, Kharkevich DA, et al. Influence of the type of surfactant on the analgesic effects induced by the peptide dalargin after its delivery across the blood-brain barrier using surfactant-coated nanoparticles. $J$ Controlled Release. 1997;49:81-87. doi:10.1016/S0168-3659(97)00061-8

418. Hejri A, Khosravi A, Gharanjig K, et al. Optimisation of the formulation of $\beta$-carotene loaded nanostructured lipid carriers prepared by solvent diffusion method. Food Chem. 2013;141:117-123. doi:10.1016/j.foodchem.2013.02.080

419. Aditya NP, Macedo AS, Doktorovova S, et al. Development and evaluation of lipid nanocarriers for quercetin delivery: a comparative study of solid lipid nanoparticles (SLN), nanostructured lipid carriers (NLC), and lipid nanoemulsions (LNE). Food Sci Tech. 2014;59:115-121. doi:10.1016/j.lwt.2014.04.058

420. Jia D, Barwal I, Thakur S, et al. Methodology to nanoencapsulate hepatoprotective components from Picrorhiza kurroa as food supplement. Food Biosci. 2015;9:28-35. doi:10.1016/j.fbio.2014. 10.005

421. Safer A, Leporatti S, Jose J, et al. Conjugation Of EGCG And Chitosan NPs As A Novel Nano-Drug Delivery System. Int $J$ Nanomedicine. 2019;14:8033-8046. doi:10.2147/IJN.S217898

422. Hashemi FS, Farzadnia F, Aghajani A, et al. Conjugated linoleic acid loaded nanostructured lipid carrier as a potential antioxidant nanocarrier for food applications. Food Sci Nutrition. 2020;8:4185-4195. doi:10.1002/fsn3.1712

423. Lacatusu I, Mitrea E, Badea N, et al. Lipid nanoparticles based on omega-3 fatty acids as effective carriers for lutein delivery. Preparation and in vitro characterization studies. J Funct Foods. 2013;5:1260-1269. doi:10.1016/j.jff.2013.04.010

424. Shahparast Y, Eskandani M, Rajaei A, et al. Preparation, physicochemical characterization and oxidative stability of omega-3 fish oil/ $\alpha$-tocopherol-co-loaded nanostructured lipidic carriers. Adv Pharm Bulletin. 2019;9:393-400. doi:10.15171/apb.2019.046
425. Yuan Y, Li H, Liu C, et al. Fabrication and characterization of lutein-loaded nanoparticles based on zein and sophorolipid: enhancement of water solubility, stability, and bioaccessibility. $J$ Agric Food Chem. 2019;67:11977-11985. doi:10.1021/acs. jafc.9b05175

426. Dai L, Wei Y, Sun C, et al. Development of protein-polysaccharide-surfactant ternary complex particles as delivery vehicles for curcumin. Food Hydrocoll. 2018;85:75-85. doi:10.1016/j. foodhyd.2018.06.052

427. Wei Y, Yang S, Zhang L, et al. Fabrication, characterization and in vitro digestion of food grade complex nanoparticles for codelivery of resveratrol and coenzyme Q10. Food Hydrocoll. 2020;105:105791. doi:10.1016/j.foodhyd.2020.105791

428. Beloqui A, Solinís MÁ, Gascón AR, et al. Mechanism of transport of saquinavir-loaded nanostructured lipid carriers across the intestinal barrier. $J$ Controlled Release. 2013;166:115-123. doi:10.1016/j.jconrel.2012.12.021

429. Park SJ, Garcia CV, Shin GH, et al. Development of nanostructured lipid carriers for the encapsulation and controlled release of vitamin D3. Food Chem. 2017;225:213-219. doi:10.1016/j. foodchem.2017.01.015

430. Ikeuchi-Takahashi Y, Shiokawa Y, Sekita K, et al. Development of microparticles coated with poly- $\gamma$-glutamic acid to improve oral absorption of a poorly water-soluble drug. Pharm Dev Technol. 2019;24:992-1001. doi:10.1080/10837450.2019.16 21898

431. Siu FY, Ye S, Lin H, et al. Galactosylated PLGA nanoparticles for the oral delivery of resveratrol: enhanced bioavailability and in vitro anti-inflammatory activity. Int $J$ Nanomedicine. 2018;13:4133-4144. doi:10.2147/IJN.S164235

432. Mohammadi M, Pezeshki A, Mesgari Abbasi M, et al. Vitamin D3-loaded nanostructured lipid carriers as a potential approach for fortifying food beverages; in vitro and in vivo evaluation. $A d v$ Pharm Bulletin. 2017;7:61-71. doi:10.15171/apb.2017.008

433. Ha ES, Sim WY, Lee SK, et al. Preparation and evaluation of resveratrol-loaded composite nanoparticles using a supercritical fluid technology for enhanced oral and skin delivery. Antioxidants. 2019:8. doi:10.3390/antiox8110554

434. Li H, Yan L, Tang EKY, et al. Synthesis of TPGS/curcumin nanoparticles by thin-film hydration and evaluation of their anticolon cancer efficacy in vitro and in vivo. Front Pharmacol. 2019:10. doi:10.3389/fphar.2019.00769

435. Yan Y, Kim JA, Kwak MK, et al. Enhanced oral bioavailability of curcumin via a solid lipid-based self-emulsifying drug delivery system using a spray-drying technique. Biol Pharm Bull. 2011;34:1179-1186. doi:10.1248/bpb.34.1179

436. Seo S, Han H, Chun M, et al. Preparation and pharmacokinetic evaluation of curcumin solid dispersion using Solutol ${ }^{\circledR}$ HS15 as a carrier. Int $J$ Pharm. 2012;424:18-25. doi:10.1016/j. ijpharm.2011.12.051

437. Peng S, Li Z, Zou L, et al. Improving curcumin solubility and bioavailability by encapsulation in saponin-coated curcumin nanoparticles prepared using a simple $\mathrm{pH}$-driven loading method. Food Funct. 2018;9:1829-1839. doi:10.1039/C7FO01814B

438. Yao J, Zhang P, Fu Z, et al. Biocompatible green tea extractstabilised zinc nanoparticles encapsulated by poly(butyl-2-cyanoacrylate) with control release profile and antioxidative capacity. Int J Food Sci Tech. 2020;55:2981-2989. doi:10.1111/ijfs.14563

439. Mohamed Saliq A, Krishnaswami V, Janakiraman K, et al. $\alpha-$ Lipoic acid nanocapsules fortified cow milk application as a dietary supplement product for anemia. Applied Nanosci. 2020;10:2007-2023. doi:10.1007/s13204-020-01304-2

440. Piran P, Kafil HS, Ghanbarzadeh S, et al. Formulation of mentholloaded nanostructured lipid carriers to enhance its antimicrobial activity for food preservation. Adv Pharm Bulletin. 2017;7:261268. doi:10.15171/apb.2017.031 
441. Bolla PK, Kalhapure RS, Rodriguez VA, et al. Preparation of solid lipid nanoparticles of furosemide-silver complex and evaluation of antibacterial activity. J Drug Deliv Sci Technol. 2019;49:6-13. doi:10.1016/j.jddst.2018.10.035

442. Gao M, Long X, Du J, et al. Enhanced curcumin solubility and antibacterial activity by encapsulation in PLGA oily core nanocapsules. Food Funct. 2020;11:448-455. doi:10.1039/ C9FO00901A

443. Gómez-Sequeda N, Ruiz J, Ortiz C, et al. Potent and specific antibacterial activity against escherichia coli O157: h7and methicillin resistant staphylococcus aureus (MRSA) of G17 and G19 peptides encapsulated into poly-lactic-co-glycolic acid (PLGA) nanoparticles. Antibiotics. 2020:9. doi:10.3390/antibiotics90 70384

444. Liu X, Gan H, Hu C, et al. Silver sulfadiazine nanosuspensionloaded thermosensitive hydrogel as a topical antibacterial agent. Int J Nanomedicine. 2018;14:289-300. doi:10.2147/IJN.S187918

445. Alkhatib Y, Dewaldt M, Moritz S, et al. Controlled extended octenidine release from a bacterial nanocellulose/Poloxamer hybrid system. Eur J Pharm Biopharmaceutics. 2017;112:164176. doi:10.1016/j.ejpb.2016.11.025

446. Pisárčik M, Lukáč M, Jampílek J, et al. Silver nanoparticles stabilised with cationic single-chain surfactants. Structure-physical properties-biological activity relationship study. J Mol Liq. 2018;272:60-72. doi:10.1016/j.molliq.2018.09.042

447. Karimi N, Ghanbarzadeh B, Hamishehkar H, et al. Antioxidant, antimicrobial and physicochemical properties of turmeric extract-loaded nanostructured lipid carrier (NLC). Colloid Interface Sci Commun. 2018;22:18-24. doi:10.1016/j. colcom.2017.11.006

448. Chaudhary S, Chauhan P, Kumar R, et al. Toxicological responses of surfactant functionalized selenium nanoparticles: a quantitative multi-assay approach. Sci Total Environ. 2018;643:1265-1277. doi:10.1016/j.scitotenv.2018.06.296

449. Khanh LL, Truc NT, Dat NT, et al. Gelatin-stabilized composites of silver nanoparticles and curcumin: characterization, antibacterial and antioxidant study. Sci Tech Adv Mater. 2019;20:276-290. doi:10.1080/14686996.2019.1585131
450. Mokarizadeh M, Kafil HS, Ghanbarzadeh S, et al. Improvement of citral antimicrobial activity by incorporation into nanostructured lipid carriers: a potential application in food stuffs as a natural preservative. Res Pharm Sci. 2017;12:409-415. doi:10.4103/1735-5362.213986

451. Yegin Y, Perez-Lewis KL, Zhang M, et al. Development and characterization of geraniol-loaded polymeric nanoparticles with antimicrobial activity against foodborne bacterial pathogens. $J$ Food Eng. 2016;170:64-71. doi:10.1016/j. jfoodeng.2015.09.017

452. Skiba MI, Vorobyoba V. The plasma-chemical formation of polysorbate 80-coated silver nanoparticles and composite materials for water treatment. Pigment \& resin tech. 2019;48:431-438. doi:10.1108/PRT-11-2018-0114

453. Guo W, Fu Z, Zhang Z, et al. Synthesis of Fe3O4 magnetic nanoparticles coated with cationic surfactants and their applications in $\mathrm{Sb}(\mathrm{V})$ removal from water. Sci Total Environ. 2020;710:136302. doi:10.1016/j.scitotenv.2019.136302

454. Pilolli R, Visconti A, Monaci L. Rapid and label-free detection of egg allergen traces in wines by surface plasmon resonance biosensor. Anal Bioanal Chem. 2015;407:3787-3797. doi:10.1007/ s00216-015-8607-4

455. Cherpinski A, Gozutok M, Sasmazel HT, et al. Electrospun Oxygen scavenging films of poly(3-hydroxybutyrate) containing palladium nanoparticles for active packaging applications. Nanomaterials. 2018;8:469. doi:10.3390/nano8070469

456. Fang L, Wang T, Lamsal B. Synergistic effect of surfactants and silica nanoparticles on oil recovery from condensed corn distillers solubles (CCDS). Ind Crops Prod. 2015;77:553-559. doi:10.1016/j.indcrop.2015.09.031
International Journal of Nanomedicine

\section{Publish your work in this journal}

The International Journal of Nanomedicine is an international, peerreviewed journal focusing on the application of nanotechnology in diagnostics, therapeutics, and drug delivery systems throughout the biomedical field. This journal is indexed on PubMed Central, MedLine, CAS, SciSearch ${ }^{\circledR}$, Current Contents ${ }^{\circledR} /$ Clinical Medicine,
Dovepress

Journal Citation Reports/Science Edition, EMBase, Scopus and the Elsevier Bibliographic databases. The manuscript management system is completely online and includes a very quick and fair peer-review system, which is all easy to use. Visit http://www.dovepress.com/ testimonials.php to read real quotes from published authors. 